\title{
ON ONE VERSION OF A SEMIDISCRETE GALERKIN METHOD FOR PDE PROBLEMS INVOLVING A GENERALIZED 2D HAMILTONIAN OPERATOR*
}

\author{
ALEXANDER ZLOTNIK ${ }^{\dagger}$, BERNARD DUCOMET ${ }^{\ddagger}$, HELOISE GOUTTE $^{\S}$, AND \\ JEAN-FRANCOIS BERGER
}

\begin{abstract}
We consider a boundary value problem, an eigenvalue problem and an initialboundary value problem involving a generalized 2D Hamiltonian operator (i.e., the second order self-adjoint elliptic operator) in a rectangular domain. We apply a semi-discrete Galerkin method exploiting space approximations of the form $c_{1}\left(x_{1}\right) \chi_{1}\left(x_{1}, x_{2}\right)+\cdots+c_{N}\left(x_{1}\right) \chi_{N}\left(x_{1}, x_{2}\right)$, where $\chi_{1}, \ldots, \chi_{N}$ are some known complex-valued basis functions and $c_{1}, \ldots, c_{N}$ are unknown coefficients. The corresponding approximate problems are stated and their properties such as existence and uniqueness of solutions and bounds for them, positive definiteness of the related sesquilinear forms, etc. are analyzed. For a specific physically reasonable choice of the basis functions, error bounds of arbitrarily high orders are proved for all the listed problems.
\end{abstract}

Key words. Elliptic equation, boundary value problem, eigenvalue problem, generalized timedependent Schrödinger equation, semi-discrete method, Galerkin method, error bounds.

AMS subject classifications. 65N30, 65M60, 81V35.

\section{Introduction}

The description of large amplitude collective motion in atomic nuclei, such as large-scale collective oscillations and nuclear fission remains one of the most challenging problems in contemporary nuclear physics. One way of tackling this kind of problem is to employ the so-called Generator Coordinate Method (GCM) [11]. This approach consists in first defining a set of static nuclear wave-functions $\Phi_{\mathbf{q}}\left(\vec{r}_{1}, \vec{r}_{2}, \ldots, \vec{r}_{A}\right)$ labeled by the composite index $\mathbf{q}=\left(q_{1}, q_{2}, \ldots, q_{M}\right)$, representing the different kinds of shapes the system is expected to adopt during time-evolution. These wave-functions usually are determined by means of self-consistent mean-field Hartree-Fock-like techniques using $M$ constraints of the form $q_{l}=\left\langle\Phi_{\mathbf{q}}\left|\hat{Q}_{l}\right| \Phi_{\mathbf{q}}\right\rangle$. In most cases the operators $\hat{Q}_{l}$ are taken as the usual multipole operators of order $l$. The time-dependent wavefunction of the system is then assumed to be the superposition of all static states $\Phi_{\mathbf{q}}$ weighted by a probability amplitude $f(q ; t)$ :

$$
\Psi\left(\vec{r}_{1}, \vec{r}_{2}, \ldots, \vec{r}_{A} ; t\right)=\int f(q ; t) \Phi_{\mathbf{q}}\left(\vec{r}_{1}, \vec{r}_{2}, \ldots, \vec{r}_{A}\right) d^{M} q
$$

The justification for this form of nuclear wave-function is similar to the BohrOppenheimer approximation of molecular physics. It is assumed that the "collective" degrees of freedom described by the set q evolve with characteristic times $\tau_{\text {coll }}$ that are much larger than the one associated with the internal motion of nucleons: $\tau_{\text {coll }} \gg \tau_{\text {int }} \simeq 10^{-23} \mathrm{~s}$. The GCM is therefore expected to be a good approximation in the low-energy regime $(E \lesssim 15 \mathrm{MeV})$.

*Received: January 23, 2008; accepted (in revised version): November 24, 2008. Communicated by Francois Golse.

${ }^{\dagger}$ Department of Applied Mathematics, Russian State Social University, W. Pieck 4, 129226 Moscow, Russia (azlotnik2008@gmail.com).

${ }^{\ddagger}$ CEA, DAM, DIFF,F-91297 Arpajon, France (bernard.ducomet@cea.fr).

$\S$ CEA, DAM, DIFF,F-91297 Arpajon, France (heloise.goutte@cea.fr).

๑CEA, DAM, DIFF,F-91297 Arpajon, France (jean-francois.berger@cea.fr). 
An equation for the unknown function $f$ in equation (1.1) is found by applying a time-dependent Schrödinger variational principle to the trial wave function $\Psi$. This procedure leads to an integro-differential equation, the so-called Hill-Wheeler (HW) equation, which is first order in time and contains a $M$-fold integral [9]. This equation is quite difficult to solve numerically as soon as $M>1$. For these reason, further approximations are made - Gaussian dependence in $\left\|\mathbf{q}-\mathbf{q}^{\prime}\right\|$ of the the overlap kernel $\left\langle\Phi_{\mathbf{q}} \mid \Phi_{\mathbf{q}^{\prime}}\right\rangle$ and expansion up to second order in the non locality $\left\|\mathbf{q}-\mathbf{q}^{\prime}\right\|$ of the Hamiltonian kernel $\left\langle\Phi_{\mathbf{q}}|\hat{H}| \Phi_{\mathbf{q}^{\prime}}\right\rangle$ - which allow one to transform the HW equation into the time-dependent Schrödinger-like equation [20]

$$
\imath \frac{\partial}{\partial t} v(\mathbf{q}, t)=\mathcal{H}^{(M)} v(\mathbf{q}, t)
$$

where $\mathcal{H}^{(M)}$ is the Hamiltonian, a linear differential operator with variable coefficients, defined as

$$
\mathcal{H}^{(M)}=-\sum_{i, j=1}^{M} \frac{\partial}{\partial q_{i}}\left(B_{i j}(\mathbf{q}) \frac{\partial}{\partial q_{j}}\right)+V(\mathbf{q}),
$$

containing inertia coefficients $B_{i j}(\mathbf{q})$ and a potential term $V(\mathbf{q})$ which are known functions of $q_{1}, q_{2}, \ldots, q_{M}$, and $\imath$ is the imaginary unit. The function $v$ is related to $f$ through convolution with a Gaussian.

Equation (1.2) has been used for instance to analyze the problem of multidimensional collective tunneling $[10,19]$. Employed with an appropriate initial condition $v(\mathbf{q}, t=0)=v_{0}(\mathbf{q})$, this equation is also the basis of present-day descriptions of the fission phenomenon $[2,8]$. A stationary version of the GCM can be derived in the same way as above by leaving aside the time-dependance in equation (1). Equation (2) then is replaced by the stationary Schrödinger-like equation

$$
\mathcal{H}^{(M)} v_{a}(\mathbf{q})=E_{a} v_{a}(\mathbf{q}),
$$

which is widely used to calculate bound states of collective nature in nuclei $[14,18$, $17]$.

Although many numerical methods exist to solve such problems, physical insights into the obtained solutions can be gained by attempting to expand them on a set of stationary functions which solve a Schrödinger equation of type (1.3) in a reduced collective space of dimension $M^{\prime}<M$. The interesting features of this technique are twofold. First, the stationary functions in $M^{\prime}$-dimensional space and their associated eigenvalues give physical information about the structure of the collective space spanned by the variables $q_{1}, \ldots, q_{M^{\prime}}$. Second, one expects from physical arguments that only a small number $N$ of these functions - those associated with the lower eigenvalues - are useful in the expansion of the full function $v$, which clearly reduces the numerical effort necessary for solving equation (2). This technique has been employed in a simple case in [19].

In principle the above method is expected to be particularly interesting when $M$ is large, but we will restrict ourselves in the present paper to the case $M=2$, which is currently used in applications. In this case, the method amounts to analyzing techniques which exploit expansions of the form

$$
v^{(N)}\left(q_{1}, q_{2}\right)=c_{1}\left(q_{1}\right) \chi_{1}\left(q_{1}, q_{2}\right)+\cdots+c_{N}\left(q_{1}\right) \chi_{N}\left(q_{1}, q_{2}\right) .
$$


In this expansion, $\chi_{1}, \ldots, \chi_{N}$ are basis functions obtained by solving a 1D stationary equation (3) in the variable $q_{2}$ for every value of $q_{1}$, and $c_{1}, \ldots, c_{N}$ are unknown coefficients. Such an expansion can be applied in the Galerkin method as well as in various other approximate methods. There exists an indirect relation to the spectral Galerkin methods, in particular see $[4,5,10]$.

In the present paper, we consider three problems involving the generalized 2D Schrödinger operator, i.e., the general variable-coefficient second-order self-adjoint elliptic operator:

(1) the boundary-value problem (BVP) with zero Dirichlet boundary conditions;

(2) the corresponding eigenvalue problem;

(3) the corresponding initial-boundary value problem (IBVP) for the associated time-dependent Schrödinger equation.

Actually, these statements are only simplifications of practically interesting problems in nuclear physics. Moreover, problem (1) is not physically relevant, but we begin with it and study it in detail for mathematical reasons.

We investigate the semi-discrete Galerkin method relying on approximations (1.4) and identify the arising approximate problems as:

(1) a boundary-value problem for a system of second-order ordinary differential equations (ODEs) for $c_{1}, \ldots, c_{N}$ with zero boundary values;

(2) the corresponding ODE eigenvalue problem;

(3) the corresponding initial-boundary value problem for the time-dependent Schrödinger-like system of 1D (in space) equations.

The paper is organized as follows. In section 2, we recall some functional spaces, state the self-adjoint elliptic BVP and list some of its basic properties. We also introduce our version of the semi-discrete Galerkin method in several equivalent forms. In section 3, we define two Gram matrices for the system of the basis functions, bound their elements, and prove uniform in space positive definiteness. As a consequence, we obtain that our infinite dimensional space of trial functions (1.4) is closed which leads to the existence and uniqueness of the approximate solution of the elliptic BVP. We also derive a BVP for a system of ODEs to define the unknown coefficients $c_{1}, \ldots, c_{N}$, bound entries of involved matrices, and study positive definiteness of the related Hermitian symmetric sesquilinear form. Results on the existence and uniqueness of weak and strong solutions of this Galerkin BVP are also presented, and an alternative form of the system of ODEs is discussed. At the end of the section, we briefly show how the version of the Galerkin method can be generalized to the multidimensional case.

Section 4 is concerned with a specific choice of the basis functions as eigenfunctions of an auxiliary 1D eigenvalue ODE problem with coefficients depending both on $x_{1}$ and $x_{2}$; in this case, the method can be considered as a Fourier-Galerkin semi-discrete method. The basic properties of this problem are recalled. We study approximation properties of the corresponding space of trial functions and prove the $L^{2}$-error bounds of optimal order $O\left(N^{-\theta}\right), \theta \geq 0$, and the much more delicate $H^{1}$-error bounds of the logarithmically optimal order $O\left(N^{-(\theta-1)}\left(1+\log ^{1 / 2} N\right)\right)$, where $\theta>1$ is the (arbitrarily high) order of smoothness of an approximating function. These bounds imply $L^{2}$ and $H^{1}$-error bounds for the semi-discrete Galerkin method for the BVP of orders $O\left(N^{-\theta}(1+\log N)\right)$ and $O\left(N^{-(\theta-1)}\left(1+\log ^{1 / 2} N\right)\right), \theta>1$. 
An example of further discretization in $x_{1}$ is considered in section 5 . The finite element method with the simplest linear elements and numerical integration is applied. We show that this leads to a finite difference scheme in $x_{1}$ for the Galerkin BVP for $c_{1}, \ldots, c_{N}$, bouns entries of involved matrices, and study positive definiteness properties of the corresponding mesh Hermitian symmetric sesquilinear form.

Section 6 deals with a 2D elliptic eigenvalue problem. We recall its basic properties, apply our version of the semi-discrete Galerkin method, and present similar properties for the approximate eigenvalue problem. The latter is the eigenvalue problem for the Galerkin system of ODEs derived in section 3. For the specific choice of the basis functions, we prove error bounds for the approximate eigenvalues of order $O\left(N^{-2(\theta-1)}(1+\log N)\right), \theta>1$, together with $L^{2}$ - and $H^{1}$-error bounds for the approximate eigenfunctions of the same orders as for the BVP.

The final section 7 is devoted to the time-dependent generalized Schrödinger equation once again 2D in space. For a weak solution of the corresponding IBVP, we recall the conservation laws, the Fourier expansion with respect to the eigenfunctions introduced in section 6 , as well as the existence and uniqueness properties. We apply our version of the semi-discrete Galerkin method and prove its similar properties. The uniform in time $L^{2}$ and $H^{1}$-error bounds of the same orders as for the BVP are also proved. All our error estimates seem to be logarithmically optimal.

2. A 2D elliptic boundary-value problem and its semi-discrete Galerkin approximation

We first recall some function spaces for the reader's convenience. For a parallelepiped $U=\left(0, X_{1}\right) \times \cdots \times\left(0, X_{n}\right)$ in $\mathbb{R}^{n}, n \geq 1$, we exploit the standard (complex) Lebesgue spaces $L^{q}(U)$, and the (complex) Sobolev spaces $H^{m}(U), m=1,2$, which are equipped with the norms

$$
\begin{gathered}
\|w\|_{L^{q}(U)}:=\left[\int_{U}|w(\xi)|^{q} d \xi\right]^{1 / q} \text { for } 1 \leq q<\infty, \quad\|w\|_{L^{\infty}(U)}:=\operatorname{ess}_{\sup _{\xi \in U}}|w(\xi)|, \\
\|w\|_{H^{1}(U)}:=\left(\|w\|_{L^{2}(U)}^{2}+\|D w \mid\|_{L^{2}(U)}^{2}\right)^{1 / 2}
\end{gathered}
$$

and

$$
\|w\|_{H^{2}(U)}:=\left(\|w\|_{H^{1}(U)}^{2}+\left\|\left|D^{2} w\right|\right\|_{L^{2}(U)}^{2}\right)^{1 / 2}
$$

Here $D w=\left(D_{1} w, \ldots, D_{n} w\right)$ and $D^{2} w=\left\{D_{i} D_{j} w\right\}_{i, j=1}^{n}$ are the gradient and the Hessian of the function $w$ (the derivatives are understood in the Sobolev sense), and $|D w|$ and $\left|D^{2} w\right|$ are their norms in $\mathbb{R}^{n}$ and $\mathbb{R}^{n \times n}$. In the proofs, the simplified notation $\|\cdot\|_{U}=\|\cdot\|_{L^{2}(U)}$ is adopted.

We also need the subspaces $H_{0}^{1}(U):=\left\{w \in H^{1}(U) ;\left.w\right|_{\partial U}=0\right\}$ and $H^{2} \cap H_{0}^{1}(U):=$ $H^{2}(U) \cap H_{0}^{1}(U)$, which can be equipped with the simpler but equivalent norms

$$
\|w\|_{H^{1}(U)}:=\||D w|\|_{L^{2}(U)}, \quad\|w\|_{H^{2} \cap H_{0}^{1}(U)}:=\left\|\left|D^{2} w\right|\right\|_{L^{2}(U)} .
$$

We exploit the dual space $H^{-1}(U):=\left[H_{0}^{1}(U)\right]^{*}$ equipped with the norm $\|f\|_{H^{-1}(U)}:=$ $\sup _{\|\varphi\|_{H_{0}^{1}(U)}=1}\left|\langle f, \varphi\rangle_{U}\right|$, where $\langle f, \varphi\rangle_{U}$ is the duality bracket on $H^{-1}(U) \times H_{0}^{1}(U)$ (generalizing the integral $\int_{U} f \varphi^{*} d \xi$ for $f, \varphi \in L^{2}(U)$, with $\varphi^{*}$ denoting the complex conjugate of $\varphi$ ). The Sobolev spaces in use are separable Hilbert spaces. $C(\bar{U})$ is the space of continuous functions on $\bar{U}$ equipped with the norm $\|w\|_{C(\bar{U})}:=\max _{\xi \in \bar{U}}|w(\xi)|$. 
For $n=2$, we use the more general anisotropic Lebesgue spaces $L^{q, r}(U)$ and $\widetilde{L}^{r, q}(U)[6,15]$, for $q, r \in[1, \infty]$, and an anisotropic Sobolev space $H^{1,0}(U)$, equipped with the norms

$$
\begin{gathered}
\|w\|_{L^{q, r}(U)}:=\|\| w\left(\cdot, \xi_{2}\right)\left\|_{L^{q}\left(I_{1}\right)}\right\|_{L^{r}\left(I_{2}\right)},\|w\|_{\widetilde{L}^{r, q}(U)}:=\|\| w\left(\xi_{1}, \cdot\right)\left\|_{L^{r}\left(I_{2}\right)}\right\|_{L^{q}\left(I_{1}\right)}, \\
\|w\|_{H^{1,0}(U)}:=\left(\|w\|_{L^{2}(U)}^{2}+\left\|D_{1} w\right\|_{L^{2}(U)}^{2}\right)^{1 / 2},
\end{gathered}
$$

where $I_{1}:=\left(0, X_{1}\right)$ and $I_{2}:=\left(0, X_{2}\right)$. It is known that $\|w\|_{\widetilde{L}^{r, q}(U)} \leq\|w\|_{L^{q, r}(U)}$ for $1 \leq r \leq q \leq \infty$.

We first consider the self-adjoint elliptic equation in a rectangle

$$
\mathcal{H} u:=-\sum_{i, j=1}^{2} D_{i}\left(\kappa_{i j} D_{j} u\right)+V u=f \text { in } \Omega,
$$

where $\Omega:=I_{1} \times I_{2}$, supplemented with the zero Dirichlet boundary conditions

$$
\left.u\right|_{\partial \Omega}=0 .
$$

We assume that the coefficients of the Hamiltonian operator $\mathcal{H}$ are real and satisfy the conditions

$$
\max _{i, j}\left\|\kappa_{i j}\right\|_{L^{\infty}(\Omega)}+\|V\|_{L^{p}(\Omega)} \leq \mu_{0} \text { for some } p \in(1,2], \kappa_{12}=\kappa_{21}, V \geq 0 .
$$

For the first condition, we automatically assume that $\kappa_{i j} \in L^{\infty}(\Omega), V \in L^{p}(\Omega)$; a similar agreement is adopted in subsequent conditions as well. We also impose the uniform in $\Omega$ ellipticity condition

$$
\nu\left(\xi_{1}^{2}+\xi_{2}^{2}\right) \leq \sum_{i, j=1}^{2} \kappa_{i j}(x) \xi_{i} \xi_{j} \text { for almost all (a.a.) } x=\left(x_{1}, x_{2}\right) \in \Omega \text { and all } \xi_{1}, \xi_{2} \in \mathbb{R},
$$

with some $\nu>0$. Let $\varkappa=\left\{\kappa_{i j}\right\}_{i, j=1}^{2}$ be the matrix of the leading coefficients of $\mathcal{H}$. Note that $V$ could also be complex-valued. However, we do not consider this possibility hereafter.

By definition, a weak solution $u \in H_{0}^{1}(\Omega)$ of the BVP (2.1), (2.2) satisfies the integral identity

$$
\mathcal{L}_{\Omega}(u, \varphi):=\int_{\Omega}\left(\sum_{i, j=1}^{2} \kappa_{i j} D_{j} u \cdot D_{i} \varphi^{*}+V u \varphi^{*}\right) d x=\langle f, \varphi\rangle_{\Omega} \text { for any } \varphi \in H_{0}^{1}(\Omega) .
$$

Hereafter we use abbreviations such as $D_{j} u \cdot w:=\left(D_{j} u\right) w$ in order to avoid extra brackets. The sesquilinear form $\mathcal{L}_{\Omega}(u, \varphi)$ is bounded and Hermitian symmetric on $H^{1}(\Omega) \times H^{1}(\Omega)$, and the associated quadratic form is positive definite on $H_{0}^{1}(\Omega)$, i.e.,

$$
\nu\|w\|_{H_{0}^{1}(\Omega)}^{2} \leq \mathcal{L}_{\Omega}(w, w) \text { for any } w \in H_{0}^{1}(\Omega) .
$$

We denote the energy norm in $H_{0}^{1}(\Omega)$ by $\|w\|_{\mathcal{E}(\Omega)}:=\left[\mathcal{L}_{\Omega}(w, w)\right]^{1 / 2}$, which is equivalent to the original one:

$$
\sqrt{\nu}\|w\|_{H_{0}^{1}(\Omega)} \leq\|w\|_{\mathcal{E}(\Omega)} \leq K_{1}\|w\|_{H_{0}^{1}(\Omega)} \text { for any } w \in H_{0}^{1}(\Omega),
$$


where $K_{1}=K_{1}\left(\mu_{0}, p, \Omega\right)$. This means that $K_{1}$ depends only on $\mu_{0}, p$, and $\Omega$ (i.e., on $X_{1}$ and $\left.X_{2}\right)$; a similar agreement is adopted throughout the paper.

It is well known (for example see $[7,13,14]$ ) that for any $f \in H^{-1}(\Omega)$, there exists a unique weak solution $u \in H_{0}^{1}(\Omega)$ which satisfies the bound

$$
\|u\|_{H_{0}^{1}(\Omega)} \leq \nu^{-1}\|f\|_{H^{-1}(\Omega)} .
$$

Moreover, under more restrictive conditions on the coefficients,

$$
\max _{i, j}\left(\left\|\kappa_{i j}\right\|_{L^{\infty}(\Omega)}+\left\|D_{i} \kappa_{i j}\right\|_{L^{\infty}(\Omega)}\right)+\|V\|_{L^{2}(\Omega)} \leq \mu_{1}
$$

and for any $f \in L^{2}(\Omega), u$ is in fact a strong solution which belongs to $H^{2} \cap H_{0}^{1}(\Omega)$, satisfies equation $(2.1)$ in $L^{2}(\Omega)$, and obeys the $H^{2}(\Omega)$ bound

$$
\|u\|_{H^{2}(\Omega)} \leq K_{2}\left(\nu, \mu_{1}, \Omega\right)\|f\|_{L^{2}(\Omega)} .
$$

Let the basis functions (generally complex-valued) $\chi_{\ell} \in H^{1}(\Omega),\left.\chi_{\ell}\right|_{x_{2}=0, X_{2}}=0$ be given for all $\ell \geq 1$ (note that we do not suppose that $\left.\chi_{\ell}\right|_{x_{1}=0, X_{1}}=0$ ). We assume that the system $\left\{\chi_{\ell}\left(x_{1}, \cdot\right)\right\}_{\ell=1}^{N}$ is linearly independent on $I_{2}$ for any $x_{1} \in \bar{I}_{1}$ (for any $N \geq 1$ ).

In this respect we recall the well known embedding inequality

$$
\|w\|_{C\left(\bar{I}_{1} ; L^{2}\left(I_{2}\right)\right)} \leq c(\Omega)\|w\|_{H^{1,0}(\Omega)} \text { for any } w \in H^{1,0}(\Omega),
$$

so that $w\left(x_{1}, \cdot\right) \in L^{2}\left(I_{2}\right)$ is well defined for any $x_{1} \in \bar{I}_{1}$. Hereafter, for any Banach space $\mathcal{B}\left(I_{2}\right)$ of functions defined on $I_{2}, C\left(\bar{I}_{1} ; \mathcal{B}\left(I_{2}\right)\right)$ denotes the Banach space of continuous functions $w: \bar{I}_{1} \rightarrow \mathcal{B}\left(I_{2}\right)$ equipped with the norm $\|w\|_{C\left(\bar{I}_{1} ; \mathcal{B}\left(I_{2}\right)\right)}:=\max _{x_{1} \in \bar{I}_{1}}\left\|w\left(x_{1}, \cdot\right)\right\|_{\mathcal{B}\left(I_{2}\right)}$. The generic constant $c(\Omega)$ depends on $\Omega$ only. Notice that $\|w\|_{L^{\infty, 2}(\Omega)} \leq$ $c(\Omega)\|w\|_{H^{1,0}(\Omega)}$ as well.

We seek an approximate solution to the BVP $(2.1),(2.2)$ in the form of a finite expansion

$$
v^{(N)}\left(x_{1}, x_{2}\right)=\sum_{\ell=1}^{N} c_{\ell}\left(x_{1}\right) \chi_{\ell}\left(x_{1}, x_{2}\right) \text { on } \bar{\Omega}
$$

with unknown coefficients $c_{\ell} \in H_{0}^{1}\left(I_{1}\right)$, for $1 \leq \ell \leq N$. For such $v^{(N)}$, we straightforwardly obtain the formula

$$
\begin{gathered}
\mathcal{H} v^{(N)}=\sum_{\ell=1}^{N}-D_{1}\left[\kappa_{11} \chi_{\ell} D_{1} c_{\ell}+\left(\kappa_{11} D_{1} \chi_{\ell}+\kappa_{12} D_{2} \chi_{\ell}\right) c_{\ell}\right] \\
-D_{2}\left(\kappa_{21} \chi_{\ell}\right) \cdot D_{1} c_{\ell}-D_{2}\left(\kappa_{21} D_{1} \chi_{\ell}+\kappa_{22} D_{2} \chi_{\ell}\right) \cdot c_{\ell}+V \chi_{\ell} c_{\ell} .
\end{gathered}
$$

Generally, this equality must be understood in the sense of $H^{-1}(\Omega)$, i.e.,

$$
\begin{array}{r}
\left\langle\mathcal{H} v^{(N)}, \varphi\right\rangle_{\Omega}=\sum_{\ell=1}^{N} \int_{\Omega}\left\{\left[\kappa_{11} \chi_{\ell} D_{1} c_{\ell}+\left(\kappa_{11} D_{1} \chi_{\ell}+\kappa_{12} D_{2} \chi_{\ell}\right) c_{\ell}\right] D_{1} \varphi^{*}\right. \\
\left.+\left[\kappa_{21} \chi_{\ell} D_{1} c_{\ell}+\left(\kappa_{21} D_{1} \chi_{\ell}+\kappa_{22} D_{2} \chi_{\ell}\right) c_{\ell}\right] D_{2} \varphi^{*}+V \chi_{\ell} c_{\ell} \varphi^{*}\right\} d x \text { for any } \varphi \in H_{0}^{1}(\Omega) .
\end{array}
$$


In the case where the regularity conditions (2.8) and

$$
\chi_{\ell} \in H^{2}(\Omega), \quad c_{\ell} \in H^{2}\left(I_{1}\right) \text { for all } 1 \leq \ell \leq N
$$

are valid, equality (2.12) holds simply in the strong sense, i.e. in $L^{2}(\Omega)$.

Generally, conditions of various kinds could be imposed on the residual $\mathcal{H} v_{N}-f$ in order to obtain a system of equations defining the unknown coefficients $\left\{c_{\ell}\right\}_{\ell=1}^{N}$ in expansion (2.11) [17]. In this paper, we apply the Galerkin method. To this end, we first introduce the linear space $S_{N}$ of functions having the form of expansion (2.11), with arbitrary $c_{\ell} \in H_{0}^{1}\left(I_{1}\right), 1 \leq \ell \leq N$. Clearly $S_{N}$ is an infinite-dimensional linear subset in $H_{0}^{1}(\Omega)$ (thus $N$ is not the dimension of $S_{N}$ ) since the following inequalities hold

$$
\begin{aligned}
\left\|v^{(N)}\right\|_{H_{0}^{1}(\Omega)} & \leq\left\|\left|D_{1} \mathbf{c}\right|\right\|_{I_{1}}\||\chi|\|_{L^{\infty, 2}(\Omega)}+\||\mathbf{c}|\|_{L^{\infty}\left(I_{1}\right)}\left(\left\|\left|D_{1} \chi\left\|_{\Omega}+\right\|\right| D_{2} \chi \mid\right\|_{\Omega}\right) \\
& \leq c(\Omega)\|\mathbf{c}\|_{\left[H_{0}^{1}\left(I_{1}\right)\right]^{N}}\|\chi\|_{\left[H^{1}(\Omega)\right]^{N}}
\end{aligned}
$$

with the column vector-functions $\mathbf{c}:=\left(c_{1}, \ldots, c_{N}\right)^{T}$ and $\chi:=\left(\chi_{1}, \ldots, \chi_{N}\right)^{T}$. Actually $S_{N}$ is a closed subspace in $H_{0}^{1}(\Omega)$; see Proposition 3.3 below.

We subject $v^{(N)}$ to the Galerkin orthogonality condition

$$
\left\langle\mathcal{H} v^{(N)}-f, \varphi\right\rangle_{\Omega}=0 \text { for any } \varphi \in S_{N}
$$

Obviously, this can be rewritten as the standard Galerkin integral identity

$$
\mathcal{L}_{\Omega}\left(v^{(N)}, \varphi\right)=\langle f, \varphi\rangle_{\Omega} \text { for any } \varphi \in S_{N}
$$

On the other hand, condition (2.16) is equivalent to the collection of particular orthogonality conditions

$$
\left\langle\mathcal{H} v^{(N)}-f, \eta \chi_{k}\right\rangle_{\Omega}=0 \text { for any } \eta \in H_{0}^{1}\left(I_{1}\right), \text { with } k=1, \ldots, N,
$$

which can be also rewritten more specifically as

$$
\left\langle\left(\mathcal{H} v^{(N)}-f\right)\left(x_{1}, \cdot\right), \chi_{k}\left(x_{1}, \cdot\right)\right\rangle_{I_{2}}=0 \text { for a.a. } x_{1} \in I_{1} \text {, with } k=1, \ldots, N
$$

In general condition (2.19) is nothing but a more abstract version of (2.18), but under the regularity conditions $(2.8),(2.14)$ and for $f \in L^{2}(\Omega)$ it takes the familiar integral form

$$
\int_{I_{2}}\left(\mathcal{H} v^{(N)}-f\right)\left(x_{1}, x_{2}\right) \chi_{k}^{*}\left(x_{1}, x_{2}\right) d x_{2}=0 \text { for a.a. } x_{1} \in I_{1} \text {, with } k=1, \ldots, N .
$$

The variational form of this method is also available (see (3.9) below).

\section{Properties of the semi-discrete Galerkin approximation}

Let $\rho_{0}$ be a real weight function such that

$$
\left\|\rho_{0}\right\|_{L^{\infty}(\Omega)}+\left\|D_{1} \rho_{0}\right\|_{L^{2}(\Omega)} \leq \mu\left(\rho_{0}\right), \quad \rho_{0}(x) \geq \underline{\rho}_{0}>0 \text { in } \Omega .
$$

Let $\bar{\rho}_{0}:=\left\|\rho_{0}\right\|_{L^{\infty}(\Omega)}$. We introduce the following Gram matrix for the system $\left\{\chi_{\ell}\right\}_{\ell=1}^{N}$ :

$$
G_{0}=\left\{g_{k \ell}\right\}_{k, \ell=1}^{N}, \quad g_{k \ell}\left(x_{1}\right):=\int_{I_{2}}\left(\rho_{0} \chi \ell \chi_{k}^{*}\right)\left(x_{1}, x_{2}\right) d x_{2} \text { for } x_{1} \in \bar{I}_{1} \text {. }
$$


The importance of studying Gram matrices is well known in Galerkin methods [18].

Lemma 3.1. The matrix $G_{0}$ has the properties

$$
\begin{gathered}
\left\|g_{k \ell}\right\|_{H^{1}\left(I_{1}\right)} \leq c(\Omega) \mu\left(\rho_{0}\right)\left\|\chi_{k}\right\|_{H^{1}(\Omega)}\left\|\chi_{\ell}\right\|_{H^{1}(\Omega)} \quad \text { for any } 1 \leq k \leq N, 1 \leq \ell \leq N \\
G_{0}\left(x_{1}\right)=G_{0}^{*}\left(x_{1}\right) \geq \nu_{0 N} \mathbb{I} \quad \text { on } \bar{I}_{1}, \text { with } \nu_{0 N}>0
\end{gathered}
$$

where $\mathbb{I}$ is the unit matrix of order $N$.

Proof. Applying embedding (2.10), we obtain

$$
\left\|g_{k \ell}\right\|_{L^{\infty}\left(I_{1}\right)} \leq \bar{\rho}_{0}\left\|\chi_{k}\right\|_{\tilde{L}^{2, \infty}(\Omega)}\left\|\chi_{\ell}\right\|_{\tilde{L}^{2, \infty}(\Omega)} \leq c(\Omega) \bar{\rho}_{0}\left\|\chi_{k}\right\|_{H^{1}(\Omega)}\left\|\chi_{\ell}\right\|_{H^{1}(\Omega)} .
$$

Moreover, using the generalized Minkowski inequality [6], we derive

$$
\begin{aligned}
\left\|D_{1} g_{k \ell}\right\|_{I_{1}}= & \left\|\int_{I_{2}}\left[D_{1} \rho_{0} \cdot \chi_{\ell} \chi_{k}^{*}+\rho_{0}\left(D_{1} \chi_{\ell} \cdot \chi_{k}^{*}+\chi_{\ell} D_{1} \chi_{k}^{*}\right)\right] d x_{2}\right\|_{I_{1}} \\
\leq & \int_{I_{2}}\left\|D_{1} \rho_{0} \cdot \chi_{\ell} \chi_{k}^{*}+\rho_{0}\left(D_{1} \chi_{\ell} \cdot \chi_{k}^{*}+\chi_{\ell} D_{1} \chi_{k}^{*}\right)\right\|_{I_{1}} d x_{2} \\
\leq & \left\|D_{1} \rho_{0}\right\|_{\Omega}\left\|\chi_{\ell}\right\|_{L^{\infty, 4}(\Omega)}\left\|\chi_{k}\right\|_{L^{\infty, 4}(\Omega)} \\
& +\bar{\rho}_{0}\left(\left\|D_{1} \chi_{\ell}\right\|_{\Omega}\left\|\chi_{k}\right\|_{L^{\infty, 2}(\Omega)}+\left\|\chi_{\ell}\right\|_{L^{\infty, 2}(\Omega)}\left\|D_{1} \chi_{k}\right\|_{\Omega}\right) .
\end{aligned}
$$

Using the embedding $H^{1}(\Omega) \subset L^{\infty, 4}(\Omega)$ [15], we complete the proof of (3.2).

Now, we have $G_{0}=G_{0}^{*} \geq \lambda_{\min }\left[G_{0}\right] \mathbb{I}$, where $\lambda_{\min }\left[G_{0}\right]$ is the minimal eigenvalue of $G_{0}$. Since the system $\left\{\chi_{\ell}\left(x_{1}, \cdot\right)\right\}_{\ell=1}^{N}$ is linearly independent on $I_{2}$, we have $\lambda_{\min }\left[G_{0}\right]\left(x_{1}\right)>0$, for any $x_{1} \in I$. By virtue of $(3.2), g_{k \ell} \in C\left(\bar{I}_{1}\right)$ and consequently $\lambda_{\min }\left[G_{0}\right] \in C\left(\bar{I}_{1}\right)$ as well. Thus inequality (3.3) follows with $\nu_{0 N}:=\min _{\bar{I}_{1}} \lambda_{\min }\left[G_{0}\right]>$ 0 .

Corollary 3.2. $G_{0}^{-1} \in\left[H^{1}\left(I_{1}\right)\right]^{N \times N}$.

Proof. This property follows from the usual explicit formula for the entries of $G_{0}^{-1}$ by using the facts that the product of two or more functions in $H^{1}\left(I_{1}\right)$ is in $H^{1}\left(I_{1}\right)$ as well and that $\operatorname{det} G_{0} \geq \nu_{0 N}^{N}$ (recall that $\operatorname{det} G_{0}$ equals the product of all $N$ eigenvalues of $\left.G_{0}\right)$.

Proposition 3.3. $S_{N}$ is a closed subspace of $H_{0}^{1}(\Omega)$.

Moreover, if $v^{(N)} \in H_{0}^{1}(\Omega)$ has the expansion (2.11) with arbitrary $\mathbf{c}$ (not necessarily from $\left.\left[H_{0}^{1}\left(I_{1}\right)\right]^{N}\right)$, then actually $\mathbf{c} \in\left[H_{0}^{1}\left(I_{1}\right)\right]^{N}$, and an inequality of the reverse type with respect to (2.15) holds:

$$
\|\mathbf{c}\|_{\left[H_{0}^{1}\left(I_{1}\right)\right]^{N}} \leq K_{3, N}\left\|v^{(N)}\right\|_{H_{0}^{1}(\Omega)},
$$

where $K_{3, N}=K_{3}\left(\nu_{0 N},\|\chi\|_{\left[H^{1}(\Omega)\right]^{N}}, \mu\left(\rho_{0}\right), \Omega\right)$.

Proof. Let $\left\{v_{m}\right\}_{m=1}^{\infty}$ be a sequence in $S_{N}$ which is convergent in $H_{0}^{1}(\Omega)$. We denote its limit by $v^{(N)} \in H_{0}^{1}(\Omega)$. By virtue of embedding (2.10), for any $x_{1} \in \bar{I}_{1}$, the sequence $\left\{v_{m}\left(x_{1}, \cdot\right)\right\}_{m=1}^{\infty}$ converges to $v^{(N)}\left(x_{1}, \cdot\right)$ in $L^{2}\left(I_{2}\right)$, and since the system $\left\{\chi_{\ell}\left(x_{1}, \cdot\right)\right\}_{\ell=1}^{N}$ is linearly independent, we get expansion (2.11) for $v^{(N)}\left(x_{1}, x_{2}\right)$, with some $\mathbf{c}\left(x_{1}\right)$. 
Clearly $\mathbf{c}\left(x_{1}\right)$ satisfies the following system of linear algebraic equations

$$
G_{0}\left(x_{1}\right) \mathbf{c}\left(x_{1}\right)=\mathbf{y}\left(x_{1}\right) \text { on } \bar{I}_{1},
$$

where the column vector-function $\mathbf{y}=\left(y_{1}, \ldots, y_{N}\right)^{T}$ in the right hand side has entries

$$
y_{k}\left(x_{1}\right)=\int_{I_{2}}\left(\rho_{0} v^{(N)} \chi_{k}^{*}\right)\left(x_{1}, x_{2}\right) d x_{2} \text { for any } 1 \leq k \leq N .
$$

Similar to bound (3.2), we have

$$
\|\mathbf{y}\|_{\left[H_{0}^{1}\left(I_{1}\right)\right]^{N}} \leq c(\Omega) \mu\left(\rho_{0}\right)\|\chi\|_{\left[H^{1}(\Omega)\right]^{N}}\left\|v^{(N)}\right\|_{H^{1}(\Omega)} .
$$

Now, applying Corollary 3.2 we see that $\mathbf{c}=G_{0}^{-1} \mathbf{y} \in\left[H_{0}^{1}\left(I_{1}\right)\right]^{N}$. Moreover, differentiating system (3.6), we obtain the system

$$
G_{0} D_{1} \mathbf{c}=D_{1} \mathbf{y}-D_{1} G_{0} \cdot \mathbf{c} .
$$

Property (3.3) applied to both systems implies that

$$
\nu_{0 N}\left|D_{1} \mathbf{c}\right| \leq\left|D_{1} \mathbf{y}\right|+\left|D_{1} G_{0}\right||\mathbf{c}| \leq\left|D_{1} \mathbf{y}\right|+\nu_{0 N}^{-1}\left|D_{1} G_{0}\right||\mathbf{y}|,
$$

thus

$$
\nu_{0 N}\|\mathbf{c}\|_{\left[H_{0}^{1}\left(I_{1}\right)\right]^{N}} \leq\left(1+\nu_{0 N}^{-1} \sqrt{X_{1} / 2}\left\|\left|D_{1} G_{0}\right|\right\|_{I_{1}}\right)\|\mathbf{y}\|_{\left[H_{0}^{1}\left(I_{1}\right)\right]^{N}} .
$$

According to bound (3.2), we obtain

$$
\left\|\left|D_{1} G_{0}\right|\right\|_{I_{1}}=\left(\sum_{k, \ell=1}^{N}\left\|D_{1} g_{k \ell}\right\|_{I_{1}}^{2}\right)^{1 / 2} \leq c(\Omega) \mu\left(\rho_{0}\right)\|\chi\|_{\left[H^{1}(\Omega)\right]^{N}}^{2} .
$$

Invoking bound (3.8), we complete the proof.

COROLlary 3.4. For any $f \in H^{-1}(\Omega)$, there exists a unique approximate solution $v^{(N)}$, which satisfies the uniform in $N$ bound (compare with (2.7))

$$
\left\|v^{(N)}\right\|_{H_{0}^{1}(\Omega)} \leq \nu^{-1}\|f\|_{H^{-1}(\Omega)} .
$$

Proof. Due to Proposition 3.3, $S_{N}$ may be considered as a separable Hilbert space equipped with the inner product $\mathcal{L}_{\Omega}(v, \varphi)$. Thus the result follows from the Riesz-Fischer representation theorem and the Galerkin integral identity (2.17); see also property (2.5).

REMARK 3.5. In bound (3.5) (including the expression for $K_{3, N}$ ), the norms $\left\|v^{(N)}\right\|_{H_{0}^{1}(\Omega)}$ and $\|\chi\|_{\left[H^{1}(\Omega)\right]^{N}}$ may be replaced by the weaker ones $\left\|D_{1} v^{(N)}\right\|_{L^{2}(\Omega)}$ and $\|\chi\|_{\left[H^{1,0}(\Omega)\right]^{N}}$, provided that one replaces the norm $\left\|D_{1} \rho_{0}\right\|_{L^{2}(\Omega)}$ by the stronger one $\left\|D_{1} \rho_{0}\right\|_{L^{2, \infty}(\Omega)}$. In fact, we have

$$
\begin{aligned}
\left\|g_{k \ell}\right\|_{L^{1}\left(I_{1}\right)} & \leq \bar{\rho}_{0}\left\|\chi_{k}\right\|_{\Omega}\left\|\chi_{\ell}\right\|_{\Omega}, \\
\left\|D_{1} \rho_{0} \cdot \chi_{\ell} \chi_{k}^{*}\right\|_{L^{2,1}(\Omega)} & \leq\left\|D_{1} \rho_{0}\right\|_{L^{2, \infty}(\Omega)}\left\|\chi_{\ell}\right\|_{L^{\infty, 2}(\Omega)}\left\|\chi_{k}\right\|_{L^{\infty, 2}(\Omega)},
\end{aligned}
$$


and therefore (see (3.4))

$$
\left\|g_{k \ell}\right\|_{H^{1}\left(I_{1}\right)} \leq c(\Omega)\left(\bar{\rho}_{0}+\left\|D_{1} \rho_{0}\right\|_{L^{2, \infty}(\Omega)}\right)\left\|\chi_{k}\right\|_{H^{1,0}(\Omega)}\left\|\chi_{\ell}\right\|_{H^{1,0}(\Omega)} .
$$

A similar change in bound (3.8) is possible as well.

Notice that since $\mathcal{L}_{\Omega}(\cdot, \cdot)$ is a Hermitian symmetric and positive definite sesquilinear form, the Galerkin method may also be restated equivalently in the Ritz-like variational version: $v^{(N)} \in S_{N}$ and

$$
\mathcal{J}_{\Omega}\left(v^{(N)}\right)=\min _{\varphi \in S_{N}} \mathcal{J}_{\Omega}(\varphi), \text { for } \mathcal{J}_{\Omega}(w):=\frac{1}{2} \mathcal{L}_{\Omega}(w, w)-\operatorname{Re}\langle f, w\rangle_{\Omega} .
$$

$\mathcal{J}_{\Omega}(w)$ is the energy functional; hereafter $\operatorname{Re} z$ and $\operatorname{Im} z$ denote the real and imaginary parts of $z \in \mathbb{C}$.

For technical reasons (see sections 5 and 6 below), we also introduce a linear space $S_{N}^{0}$ of functions having the form of expansion (2.11) for any $c_{\ell} \in L^{2}\left(I_{1}\right)$, for $1 \leq \ell \leq N$. Note that $S_{N}^{0} \subset L^{2}(\Omega)$ due to inequality (2.10).

Proposition 3.6. $S_{N}^{0}$ is a closed subspace of $L^{2}(\Omega)$.

Proof. Let $\left\{v_{m}\right\}_{m=1}^{\infty}$ be a sequence in $S_{N}^{0}$ converging in $L^{2}(\Omega)$, and $v^{(N)} \in L^{2}(\Omega)$ be its limit. Since for a subsequence $\left\{v_{m_{k}}\right\}_{k=1}^{\infty}$ we have

$$
\left\|\left(v_{m_{k}}-v^{(N)}\right)\left(x_{1}, \cdot\right)\right\|_{I_{2}} \rightarrow 0 \text { as } k \rightarrow \infty, \text { for a.a. } x_{1} \in I_{1},
$$

the function $v^{(N)}\left(x_{1}, x_{2}\right)$ has an expansion (2.11) for some $\mathbf{c}\left(x_{1}\right)$.

Going back to system (3.6), (3.7), we observe that

$$
\||\mathbf{y}|\|_{I_{1}} \leq\left\|\rho_{0} v|\chi|\right\|_{L^{2,1}(\Omega)} \leq c(\Omega) \bar{\rho}_{0}\left\|v^{(N)}\right\|_{\Omega}\||\chi|\|_{H^{1,0}(\Omega)} .
$$

Due to Corollary 3.2, $\mathbf{c}=G_{0}^{-1} \mathbf{y} \in\left[L^{2}\left(I_{1}\right)\right]^{N}$; recall that $\||\mathbf{c}|\|_{I_{1}} \leq \nu_{0 N}^{-1}\||\mathbf{y}|\|_{I_{1}}$.

Let $(\cdot, \cdot)_{\mathbb{C}^{N}}$ be the inner product in $\mathbb{C}^{N}$.

Lemma 3.7. Let $v$ and $\varphi$ be any functions in $S_{N}$ such that

$$
v\left(x_{1}, x_{2}\right)=\sum_{\ell=1}^{N} c_{\ell}\left(x_{1}\right) \chi_{\ell}\left(x_{1}, x_{2}\right), \quad \varphi\left(x_{1}, x_{2}\right)=\sum_{\ell=1}^{N} d_{\ell}\left(x_{1}\right) \chi_{\ell}\left(x_{1}, x_{2}\right) \quad \text { in } \Omega,
$$

with $\mathbf{c}=\left(c_{1}, \ldots, c_{N}\right)^{T}, \mathbf{d}=\left(d_{1}, \ldots, d_{N}\right)^{T} \in\left[H_{0}^{1}\left(I_{1}\right)\right]^{N}$. Then the following identity holds

$$
\mathcal{L}_{\Omega}(v, \varphi)=\mathcal{L}_{I_{1}}^{N}(\mathbf{c}, \mathbf{d}):=\int_{I_{1}}\left[\left(A D_{1} \mathbf{c}+A^{(0)} \mathbf{c}, D_{1} \mathbf{d}\right)_{\mathbb{C}^{N}}+\left(A^{(0)^{*}} D_{1} \mathbf{c}+B \mathbf{c}, \mathbf{d}\right)_{\mathbb{C}^{N}}\right] d x_{1}
$$

Here the $N \times N$-matrices $A, A^{(0)}$ and $B$ have the entries that are functions on $I_{1}$, for $1 \leq k \leq N$ and $1 \leq \ell \leq N$ :

$$
\begin{aligned}
& a_{k \ell}\left(x_{1}\right)=\int_{I_{2}}\left(\kappa_{11} \chi_{k}^{*} \chi_{\ell}\right)\left(x_{1}, x_{2}\right) d x_{2}, \\
& a_{k \ell}^{(0)}\left(x_{1}\right)=\int_{I_{2}}\left[\chi_{k}^{*}\left(\kappa_{11} D_{1} \chi_{\ell}+\kappa_{12} D_{2} \chi_{\ell}\right)\right]\left(x_{1}, x_{2}\right) d x_{2}, \\
& b_{k \ell}\left(x_{1}\right)=\int_{I_{2}}\left(\sum_{i, j=1}^{2} \kappa_{i j} D_{j} \chi_{\ell} \cdot D_{i} \chi_{k}^{*}+V \chi_{k}^{*} \chi_{\ell}\right)\left(x_{1}, x_{2}\right) d x_{2} .
\end{aligned}
$$


For these entries, the following bound holds for any $1 \leq k \leq N$ and $1 \leq \ell \leq N$ :

$$
\left\|a_{k \ell}\right\|_{L^{\infty}\left(I_{1}\right)}+\left\|a_{k \ell}^{(0)}\right\|_{L^{2}\left(I_{1}\right)}+\left\|b_{k \ell}\right\|_{L^{1}\left(I_{1}\right)} \leq K_{4}\left(\mu_{0}, p, \Omega\right)\left\|\chi_{k}\right\|_{H^{1}(\Omega)}\left\|\chi_{\ell}\right\|_{H^{1}(\Omega)} .
$$

Under the regularity conditions (2.8) on $\kappa_{i j}$ and $V$ (the condition on $D_{2} \kappa_{2 j}$ may be omitted) and

$$
D D_{1} \chi_{\ell} \in\left[L^{2}(\Omega)\right]^{2} \quad \text { for } 1 \leq \ell \leq N,
$$

the following bound also holds:

$$
\begin{aligned}
& \left\|D_{1} a_{k \ell}\right\|_{L^{\infty}\left(I_{1}\right)}+\left\|D_{1} a_{k \ell}^{(0)}\right\|_{L^{2}\left(I_{1}\right)}+\left\|b_{k \ell}\right\|_{L^{2}\left(I_{1}\right)} \\
\leq & K_{5}\left(\mu_{1}, \Omega\right)\left(\left\|\chi_{k}\right\|_{H^{1}(\Omega)}+\left\|D_{1} \chi_{k}\right\|_{L^{\infty, 2}(\Omega)}\right)\left(\left\|\chi_{\ell}\right\|_{H^{1}(\Omega)}+\left\|D D_{1} \chi_{\ell}\right\| \|_{L^{2}(\Omega)}\right) .
\end{aligned}
$$

Proof. Exploiting expansions (3.10) and rearranging the summands, we obtain

$$
\begin{aligned}
\mathcal{L}_{\Omega}(v, \varphi)= & \int_{\Omega} \sum_{k, \ell=1}^{N}\left[\sum_{i, j=1}^{2} \kappa_{i j} D_{j}\left(c_{\ell} \chi_{\ell}\right) \cdot D_{i}\left(d_{k}^{*} \chi_{k}^{*}\right)+V c_{\ell} \chi_{\ell} d_{k}^{*} \chi_{k}^{*}\right] d x \\
= & \int_{I_{1}} \sum_{k, \ell=1}^{N} \int_{I_{2}}\left\{\left[\kappa_{11}\left(D_{1} c_{\ell} \cdot \chi_{\ell}+c_{\ell} D_{1} \chi_{\ell}\right)+\kappa_{12} c_{\ell} D_{2} \chi_{\ell}\right]\left(D_{1} d_{k}^{*} \cdot \chi_{k}^{*}+d_{k}^{*} D_{1} \chi_{k}^{*}\right)\right. \\
& \left.+\left[\kappa_{21}\left(D_{1} c_{\ell} \cdot \chi_{\ell}+c_{\ell} D_{1} \chi_{\ell}\right)+\kappa_{22} c_{\ell} D_{2} \chi_{\ell}\right] d_{k}^{*} D_{2} \chi_{k}^{*}+V c_{\ell} \chi_{\ell} d_{k}^{*} \chi_{k}^{*}\right\} d x_{2} d x_{1} \\
= & \int_{I_{1}} \sum_{k, \ell=1}^{N}\left[\left(a_{k \ell} D_{1} c_{\ell}+a_{k \ell}^{(0)} c_{\ell}\right) D_{1} d_{k}^{*}+\left(a_{\ell k}^{(0)}{ }^{*} D_{1} c_{\ell}+b_{k \ell}^{(0)} c_{\ell}\right) d_{k}^{*}\right] d x_{1},
\end{aligned}
$$

where $a_{k \ell}, a_{k \ell}^{(0)}$ and $b_{k \ell}^{(0)}$ are given by the above formulas (3.12)-(3.14). Identity (3.11) is proved.

Bound (3.15) follows from the inequalities

$$
\begin{aligned}
\left\|a_{k \ell}\right\|_{L^{\infty}\left(I_{1}\right)} & \leq\left\|\kappa_{11} \chi_{k}^{*} \chi_{\ell}\right\|_{L^{\infty, 1}(\Omega)} \leq\left\|\kappa_{11}\right\|_{L^{\infty}(\Omega)}\left\|\chi_{k}\right\|_{L^{\infty, 2}(\Omega)}\left\|\chi_{\ell}\right\|_{L^{\infty, 2}(\Omega)} \\
\left\|a_{k \ell}^{(0)}\right\|_{L^{2}\left(I_{1}\right)} & \leq\left\|\chi_{k}^{*}\left(\kappa_{11} D_{1} \chi_{\ell}+\kappa_{12} D_{2} \chi_{\ell}\right)\right\|_{L^{2,1}(\Omega)} \\
& \leq\left(\left\|\kappa_{11}\right\|_{L^{\infty}(\Omega)}+\left\|\kappa_{12}\right\|_{L^{\infty}(\Omega)}\right)\left\|\chi_{k}\right\|_{L^{\infty, 2}(\Omega)}\left\|D \chi_{\ell}\right\|_{\Omega}, \\
\left\|b_{k \ell}\right\|_{L^{1}\left(I_{1}\right)} & \leq\left\|2 \mu _ { 0 } \left|D \chi_{k}\left\|D \chi_{\ell}|+V| \chi_{k} \chi \ell\right\|_{L^{1}(\Omega)}\right.\right. \\
& \leq 2 \mu_{0}\left\|D \chi_{k}\right\|_{\Omega}\left\|D \chi_{\ell}\right\|_{\Omega}+\|V\|_{L^{p}(\Omega)}\left\|\chi_{k}\right\|_{L^{2 p^{\prime}}(\Omega)}\left\|\chi_{\ell}\right\|_{L^{2 p^{\prime}}(\Omega)}
\end{aligned}
$$

and the embedding $H^{1}(\Omega) \subset L^{q}(\Omega)$ for any $1 \leq q<\infty$. Under the regularity condition (2.8) and (3.16), we also have

$$
\begin{aligned}
&\left\|D_{1} a_{k \ell}\right\|_{L^{\infty}\left(I_{1}\right)} \leq\left\|D_{1} \kappa_{11}\right\|_{L^{\infty}(\Omega)}\left\|\chi_{k}\right\|_{L^{\infty, 2}(\Omega)}\left\|\chi_{\ell}\right\|_{L^{\infty, 2}(\Omega)} \\
&+\mu_{0}\left(\left\|D_{1} \chi_{k}\right\|_{L^{\infty, 2}(\Omega)}\left\|\chi_{\ell}\right\|_{L^{\infty, 2}(\Omega)}+\left\|\chi_{k}\right\|_{L^{\infty, 2}(\Omega)}\left\|D_{1} \chi_{\ell}\right\|_{L^{\infty, 2}(\Omega)}\right), \\
&\left\|D_{1} a_{k \ell}^{(0)}\right\|_{I_{1}} \leq\left\|D_{1} \chi_{k}\right\|_{L^{\infty, 2}(\Omega)} \sqrt{2} \mu_{0}\left\|\mid D \chi_{\ell}\right\| \|_{\Omega} \\
&+\left\|\chi_{k}\right\|_{L^{\infty, 2}(\Omega)}\left[\left(\left\|D_{1} \kappa_{11}\right\|_{L^{\infty}(\Omega)}+\left\|D_{1} \kappa_{12}\right\|_{L^{\infty}(\Omega)}\right)\left\|D \chi_{\ell}\left|\left\|_{\Omega}+\mu_{0}\right\| D D_{1} \chi_{\ell}\right|\right\|_{\Omega}\right], \\
&\left\|b_{k \ell}\right\|_{I_{1}} \leq 2 \mu_{0}\left\|\left|D \chi_{\ell}\right|\right\|_{L^{\infty, 2}(\Omega)}\left\|D \chi_{k} \mid\right\|_{\Omega}+\|V\|_{\Omega}\left\|\chi_{k}\right\|_{L^{\infty, 2}(\Omega)}\left\|\chi_{\ell}\right\|_{L^{\infty}(\Omega)},
\end{aligned}
$$


thus obtaining (3.17).

COROLlary 3.8. $\quad \mathcal{L}_{I_{1}}^{N}(\cdot, \cdot)$ is a bounded Hermitian symmetric sesquilinear form on $\left[H_{0}^{1}(\Omega)\right]^{N} \times\left[H_{0}^{1}(\Omega)\right]^{N}$, with the self-adjoint matrices $A$ and $B$.

Proof. $\quad \mathcal{L}_{I_{1}}^{N}(\mathbf{c}, \mathbf{d})$ is a Hermitian symmetric sesquilinear form since $\mathcal{L}_{\Omega}(v, \varphi)$ is. The former is also bounded because of bound (3.15).

Since the matrix $\varkappa$ is symmetric (and real together with $V$ ), $A$ and $B$ are selfadjoint.

REMARK 3.9. In general the basis functions in the system $\left\{\chi_{\ell}\right\}_{\ell=1}^{N}$ can depend on $N$. If they do not, then additional rows and columns appear in the $N \times N$-matrices $A$, $A^{(0)}$ and $B$ as $N$ increases but no change appears in the previous rows and columns.

We observe that the role of the coefficients $\kappa_{i j}$ is not the same in $\mathcal{L}_{I_{1}}^{N}(\cdot, \cdot)$. The leading matrix $A$ only depends on $\kappa_{11}$, and $A^{(0)}$ and $B$ depend on $\kappa_{11}$ as well whereas only $B$ depends on $\kappa_{22}$ (and $V$ ) together with other $\kappa_{i j}$.

Now we study positive definiteness of the involved matrices and the sesquilinear form $\mathcal{L}_{I_{1}}^{N}(\cdot, \cdot)$.

Proposition 3.10. The matrices $A\left(x_{1}\right)$ and $B\left(x_{1}\right)$ are positive definite for a.a. $x_{1} \in I_{1}$, uniformly with respect to $x_{1}$. More precisely, for a.a. $x_{1} \in I_{1}$, the following matrix inequalities hold:

$$
\begin{aligned}
& \frac{\nu \nu_{0 N}}{\bar{\rho}_{0}} \mathbb{I} \leq \frac{\nu}{\bar{\rho}_{0}} G_{0}\left(x_{1}\right) \leq \frac{1}{\left\|\left(\rho_{0} / \kappa_{11}\right)\left(x_{1}, \cdot\right)\right\|_{L^{\infty}\left(I_{2}\right)}} G_{0}\left(x_{1}\right) \leq A\left(x_{1}\right), \\
& \frac{2 \nu \nu_{0 N}}{X_{2}^{2} \bar{\rho}_{0}} \mathbb{I} \leq \frac{2 \nu}{X_{2}^{2} \bar{\rho}_{0}} G_{0}\left(x_{1}\right) \leq \frac{2 \nu}{X_{2}\left\|\rho_{0}\left(x_{1}, \cdot\right)\right\|_{L^{1}\left(I_{2}\right)}} G_{0}\left(x_{1}\right) \leq B\left(x_{1}\right) .
\end{aligned}
$$

Proof. Let $v$ be given by the first expansion (3.10) for any $\mathbf{c} \in \mathbb{C}^{N}$ independent of $x_{1}$. Then by the ellipticity condition $(2.3)$ and the condition $V \geq 0$, we obtain

$$
\begin{aligned}
\left(A\left(x_{1}\right) \mathbf{c}, \mathbf{c}\right)_{\mathbb{C}^{N}} & =\int_{I_{2}}\left(\kappa_{11}|v|^{2}\right)\left(x_{1}, x_{2}\right) d x_{2} \\
& \geq \frac{1}{\left\|\left(\rho_{0} / \kappa_{11}\right)\left(x_{1}, \cdot\right)\right\|_{L^{\infty}\left(I_{2}\right)}} \int_{I_{2}}\left(\rho_{0}|v|^{2}\right)\left(x_{1}, x_{2}\right) d x_{2}, \\
\left(B\left(x_{1}\right) \mathbf{c}, \mathbf{c}\right)_{\mathbb{C}^{N}} & =\int_{I_{2}}\left(\sum_{i, j=1}^{N} \kappa_{i j} D_{j} v \cdot D_{i} v^{*}+V|v|^{2}\right)\left(x_{1}, x_{2}\right) d x_{2} \\
& \geq \nu \int_{I_{2}}\left(\left|D_{2} v\right|^{2}\right)\left(x_{1}, x_{2}\right) d x_{2} \\
& \geq \frac{2 \nu}{X_{2}\left\|\rho_{0}\left(x_{1}, \cdot\right)\right\|_{L^{1}\left(I_{2}\right)}} \int_{I_{2}}\left(\rho_{0}|v|^{2}\right)\left(x_{1}, x_{2}\right) d x_{2} .
\end{aligned}
$$

Applying the identity

$$
\int_{I_{2}}\left(\rho_{0}|v|^{2}\right)\left(x_{1}, x_{2}\right) d x_{2}=\left(G_{0}\left(x_{1}\right) \mathbf{c}, \mathbf{c}\right)_{\mathbb{C}^{N}} \text { on } \bar{I}_{1}
$$


and property (3.3), we complete the proof.

Proposition 3.11. The sesquilinear form $\mathcal{L}_{I_{1}}^{N}(\cdot, \cdot)$ has the following weakened positive definiteness property

$$
\frac{2 \nu \nu_{0 N}}{X_{1} \bar{\rho}_{0}}\||\mathbf{c}|\|_{C\left(\bar{I}_{1}\right)}^{2} \leq \mathcal{L}_{I_{1}}^{N}(\mathbf{c}, \mathbf{c}) \quad \text { for any } \mathbf{c} \in\left[H_{0}^{1}\left(I_{1}\right)\right]^{N} .
$$

Proof. By Lemma 3.7 and property (2.5), for any $\mathbf{c} \in\left[H_{0}^{1}\left(I_{1}\right)\right]^{N}$, we obtain

$$
\mathcal{L}_{I_{1}}^{N}(\mathbf{c}, \mathbf{c})=\mathcal{L}_{\Omega}(v, v) \geq \nu \int_{\Omega}|D v|^{2} d x,
$$

where $\mathbf{c}$ and $v \in \mathcal{S}_{N}$ are related by the first expansion (3.10). Taking into account identity (3.21), we have

$$
\begin{aligned}
\frac{X_{1}}{2} \int_{\Omega}\left|D_{1} v\right|^{2} d x & \geq \int_{I_{2}}\left|v\left(x_{1}, x_{2}\right)\right|^{2} d x_{2} \geq \frac{1}{\bar{\rho}_{0}} \int_{I_{2}}\left(\rho_{0}|v|^{2}\right)\left(x_{1}, x_{2}\right) d x_{2} \\
& =\frac{\nu}{\bar{\rho}_{0}}\left(G_{0}\left(x_{1}\right) \mathbf{c}\left(x_{1}\right), \mathbf{c}\left(x_{1}\right)\right)_{\mathbb{C}^{N}} \text { on } I_{1} .
\end{aligned}
$$

Applying property (3.3), we complete the proof.

Now we state and study the problem for the unknown coefficients in the Galerkin method (2.11), (2.17).

Proposition 3.12. For the vector-valued function $\mathbf{c}^{(N)}=\left(c_{1}, \ldots, c_{N}\right)^{T}$ built with the coefficients in the expansion (2.11) of the approximate solution $v^{(N)}$, the Galerkin integral identity (2.17) means that $\mathbf{c}^{(N)}$ is a weak solution in $\left[H_{0}^{1}\left(I_{1}\right)\right]^{N}$ of the following $B V P$ for a self-adjoint system of second order ODEs

$$
\begin{aligned}
& \mathcal{H}_{N} \mathbf{c}^{(N)}:=-D_{1}\left(A D_{1} \mathbf{c}^{(N)}+A^{(0)} \mathbf{c}^{(N)}\right)+A^{(0)^{*}} D_{1} \mathbf{c}^{(N)}+B \mathbf{c}^{(N)}=\mathbf{f}^{(N)} \text { on } I_{1}, \\
& \left.\mathbf{c}^{(N)}\right|_{x_{1}=0, X_{1}}=0
\end{aligned}
$$

i.e., $\mathbf{c}^{(N)}$ satisfies an integral identity

$$
\mathcal{L}_{I_{1}}^{N}\left(\mathbf{c}^{(N)}, \mathbf{d}\right)=\left\langle\mathbf{f}^{(N)}, \mathbf{d}\right\rangle_{I_{1}}:=\sum_{k=1}^{N}\left\langle f_{k}, d_{k}\right\rangle_{I_{1}} \quad \text { for any } \mathbf{d} \in\left[H_{0}^{1}\left(I_{1}\right)\right]^{N} .
$$

Here the generalized vector-valued function $\mathbf{f}^{(N)}=\left(f_{1}, \ldots, f_{N}\right)^{T}$ has components $f_{k} \in$ $H^{-1}\left(I_{1}\right)$ such that

$$
\left\langle f_{k}, \eta\right\rangle_{I_{1}}=\left\langle f, \eta \chi_{k}\right\rangle_{\Omega} \text { for any } \eta \in H_{0}^{1}\left(I_{1}\right) \text {, with } k=1, \ldots, N .
$$

In the case where $f \in L^{1,4 / 3}(\Omega)$ the last relation reduces to

$$
f_{k}\left(x_{1}\right)=\int_{I_{2}}\left(f \chi_{k}^{*}\right)\left(x_{1}, x_{2}\right) d x_{2} \text { for a.a. } x_{1} \in I_{1} .
$$

Proof. This result is a direct consequence of Lemma 3.7.

Remark 3.13. Clearly, the integral identity (3.26), for $\mathbf{d}$ such that $d_{k}=\eta \chi_{k}$ and $d_{\ell}=0$ for $\ell \neq k$, together with formulas (3.12)-(3.14) for the elements of the involved 
matrices can be also obtained from the orthogonality conditions (2.18) and formula (2.13) for $\mathcal{H} v^{(N)}$ with $\varphi=\eta \chi_{k}$ (by using $D_{1}\left(\eta \chi_{k}\right)=D_{1} \eta \cdot \chi_{k}+\eta D_{1} \chi_{k}$ in that formula). Moreover, under the regularity conditions (2.8) and (2.14), the system of ODEs (3.24) together with formulas (3.12)-(3.14) directly appear from the orthogonality conditions (2.20) by exploiting formula (2.12) for $\mathcal{H} v^{(N)}$.

REMARK 3.14. One can rewrite the sesquilinear form in the integral identity (3.26) as follows:

$$
\begin{aligned}
\mathcal{L}_{I_{1}}^{N}(\mathbf{c}, \mathbf{d})= & \int_{I_{1}}\left[\left(A D_{1} \mathbf{c}, D_{1} \mathbf{d}\right)_{\mathbb{C}^{N}}-2 \imath\left(\tilde{A}^{(0)} D_{1} \mathbf{c}, \mathbf{d}\right)_{\mathbb{C}^{N}}+(B \mathbf{c}, \mathbf{d})_{\mathbb{C}^{N}}\right] d x_{1} \\
& -\left\langle\left(D_{1} A^{(0)}\right) \mathbf{c}, \mathbf{d}\right\rangle_{I_{1}},
\end{aligned}
$$

where the self-adjoint matrix $\tilde{A}^{(0)}:=\frac{1}{2 \imath}\left(A^{(0)}-A^{(0)^{*}}\right)$ has the entries, for $1 \leq k \leq N$ and $1 \leq \ell \leq N$ :

$$
\tilde{a}_{k \ell}^{(0)}\left(x_{1}\right)=\frac{\imath}{2} \int_{I_{1}}\left[\kappa_{11}\left(D_{1} \chi_{k}^{*} \cdot \chi_{\ell}-\chi_{k}^{*} D_{1} \chi_{\ell}\right)+\kappa_{12}\left(D_{2} \chi_{k}^{*} \cdot \chi_{\ell}-\chi_{k}^{*} D_{2} \chi_{\ell}\right)\right] d x_{2} \text { on } I_{1} .
$$

In addition, generally

$$
\left\langle\left(D_{1} A^{(0)}\right) \mathbf{c}, \mathbf{d}\right\rangle_{I_{1}}=-\int_{I_{1}}\left[\left(A^{(0)} D_{1} \mathbf{c}, \mathbf{d}\right)_{\mathbf{C}^{N}}+\left(A^{(0)} \mathbf{c}, D_{1} \mathbf{d}\right)_{\mathbf{C}^{N}}\right] d x_{1},
$$

or simply

$$
\left\langle\left(D_{1} A^{(0)}\right) \mathbf{c}, \mathbf{d}\right\rangle_{I_{1}}=\int_{I_{1}}\left(D_{1} A^{(0)} \cdot \mathbf{c}, \mathbf{d}\right)_{\mathbb{C}^{N}} d x_{1}
$$

provided that the regularity conditions (2.8) and (3.16) hold so that $D_{1} A^{(0)} \in$ $\left[L^{2}\left(I_{1}\right)\right]^{N \times N}$ (see (3.17)). This corresponds to rewriting the system of ODEs (3.24) as

$$
-D_{1}\left(A D_{1} \mathbf{c}^{(N)}\right)-2 \imath \tilde{A}^{(0)} D_{1} \mathbf{c}^{(N)}+\left(B-D_{1} A^{(0)}\right) \mathbf{c}^{(N)}=\mathbf{f}^{(N)} \text { on } I_{1}
$$

REMARK 3.15. In the simplest case, where $N=1$, the system of ODEs (3.24) is reduced to the self-adjoint ODE

$$
-D_{1}\left(a_{11} D_{1} c_{1}+a_{11}^{(0)} c_{1}\right)+a_{11}^{(0)^{*}} D_{1} c_{1}+b_{11}^{(0)} c_{1}=f_{1} \quad \text { on } I_{1},
$$

and the coefficients and the right hand side are given by formulas (3.12)-(3.14) and (3.27) for $k=\ell=1$. After the previous remark, this ODE can also be rewritten as

$$
-D_{1}\left(a_{11} D_{1} c_{1}\right)-2 \imath\left(\operatorname{Im} a_{11}^{(0)}\right) D_{1} c_{1}+\left(b_{11}^{(0)}-D_{1} a_{11}^{(0)}\right) c_{1}=f_{1} \quad \text { on } I_{1} .
$$

Let $\kappa_{0}$ and $V_{0}$ be two auxiliary real-valued functions such that

$$
\kappa_{0} \in L^{\infty}(\Omega), \kappa_{0}\left(x_{1}, x_{2}\right) \geq \underline{\kappa}_{0}>0 \text { on } \Omega, V_{0} \in \tilde{L}^{1, \infty}(\Omega), V_{0} \geq 0 .
$$


We introduce another Gram matrix for the system $\left\{\chi_{\ell}\right\}_{\ell=1}^{N}$ as

$$
G^{(1)}=\left\{g_{k \ell}^{(1)}\right\}_{k, \ell=1}^{N}, \quad g_{k \ell}^{(1)}\left(x_{1}\right):=\int_{I_{2}}\left(\kappa_{0} D_{2} \chi_{\ell} \cdot D_{2} \chi_{k}^{*}+V_{0} \chi_{\ell} \chi_{k}^{*}\right)\left(x_{1}, x_{2}\right) d x_{2} \text { on } I_{1} .
$$

Proposition 3.16. $G^{(1)} \in\left[L^{1}\left(I_{1}\right)\right]^{N \times N}, G^{(1)}=G^{(1)^{*}}$ and the following matrix inequality holds:

$$
\frac{\nu}{K_{6}} G^{(1)}\left(x_{1}\right) \leq B\left(x_{1}\right) \text { for a.a. } x_{1} \in I_{1},
$$

where $K_{6}:=\left\|\kappa_{0}\right\|_{L^{\infty}(\Omega)}+\left(X_{2} / 2\right)\left\|V_{0}\right\|_{\tilde{L}^{1, \infty}(\Omega)}$.

Moreover, $G^{(1)} \in\left[C\left(\bar{I}_{1}\right)\right]^{N \times N}$ and the following inequality holds:

$$
G^{(1)}\left(x_{1}\right) \geq \nu_{N}^{(1)} \mathbb{I} \text { on } \bar{I}_{1} \text {, with } \nu_{N}^{(1)}>0,
$$

provided that

$$
D_{2} \chi_{\ell} \in C\left(\bar{I}_{1} ; L^{2}\left(I_{2}\right)\right) \quad \text { for } 1 \leq \ell \leq N, \kappa_{0} \in C\left(\bar{I}_{1} ; L^{\infty}\left(I_{2}\right)\right), V_{0} \in C\left(\bar{I}_{1} ; L^{1}\left(I_{2}\right)\right) .
$$

Proof. We have (compare with (3.4))

$$
\begin{aligned}
\left\|g_{k \ell}^{(1)}\right\|_{L^{1}\left(I_{1}\right)} & \leq\left\|\kappa_{0}\right\|_{L^{\infty}(\Omega)}\left\|D_{2} \chi_{\ell}\right\|_{\Omega}\left\|D_{2} \chi_{k}\right\|_{\Omega}+\left\|V_{0}\right\|_{\tilde{L}^{1, \infty}(\Omega)}\left\|\chi_{\ell}\right\|_{\tilde{L}^{\infty, 2}(\Omega)}\left\|\chi_{k}\right\|_{\tilde{L}^{\infty, 2}(\Omega)} \\
& \leq K_{6}\left\|D_{2} \chi_{\ell}\right\|_{\Omega}\left\|D_{2} \chi_{k}\right\|_{\Omega} .
\end{aligned}
$$

Let $v=\sum_{\ell=1}^{N} c_{\ell} \chi_{\ell}$, for any $\mathbf{c} \in \mathbb{C}^{N}$. Then, similar to (3.20) and the last inequality, we obtain

$$
\begin{aligned}
\left(B\left(x_{1}\right) \mathbf{c}, \mathbf{c}\right)_{\mathbb{C}^{N}} & \geq \nu \int_{I_{2}}\left(\left|D_{2} v\right|^{2}\right)\left(x_{1}, x_{2}\right) d x_{2} \geq \frac{\nu}{K_{6}} \int_{I_{2}}\left(\kappa_{0}\left|D_{2} v\right|^{2}+V_{0}|v|^{2}\right)\left(x_{1}, x_{2}\right) d x_{2} \\
& =\frac{\nu}{K_{6}}\left(G^{(1)}\left(x_{1}\right) \mathbf{c}, \mathbf{c}\right)_{\mathbb{C}^{N}} \quad \text { for a.a. } x_{1} \in I_{1}
\end{aligned}
$$

which completes the first part of the proof.

Under conditions (3.32), clearly $\chi_{\ell} \in C\left(\bar{I}_{1} ; H_{0}^{1}\left(I_{2}\right)\right)$, thus

$$
\left\|g_{k \ell}^{(1)}\right\|_{C\left(\bar{I}_{1}\right)} \leq\left(\left\|\kappa_{0}\right\|_{C\left(\bar{I}_{1} ; L^{\infty}\left(I_{2}\right)\right)}+\left\|V_{0}\right\|_{C\left(\bar{I}_{1} ; L^{1}\left(I_{2}\right)\right)}\right)\left\|\chi_{\ell}\right\|_{C\left(\bar{I}_{1} ; H_{0}^{1}\left(I_{2}\right)\right)}\left\|\chi_{k}\right\|_{C\left(\bar{I}_{1} ; H_{0}^{1}\left(I_{2}\right)\right)} .
$$

After that, the proof of inequality (3.31) follows that of (3.3).

We are now in position to derive the strong positive definiteness property of $\mathcal{L}_{I_{1}}^{N}(\cdot, \cdot)$.

Proposition 3.17. The sesquilinear form $\mathcal{L}_{I_{1}}^{N}(\cdot, \cdot)$ has the following $H_{0}^{1}\left(I_{1}\right)$-positive definiteness property

$$
\nu K_{7, N}^{-1}\|\mathbf{c}\|_{\left[H_{0}^{1}\left(I_{1}\right)\right]^{N}}^{2} \leq \mathcal{L}_{I_{1}}^{N}(\mathbf{c}, \mathbf{c}) \text { for any } \mathbf{c} \in\left[H_{0}^{1}(\Omega)\right]^{N},
$$

where $K_{7, N}=K_{3, N}^{2}\left(\right.$ see (3.5)) or $K_{7, N}=K_{8, N}$ with

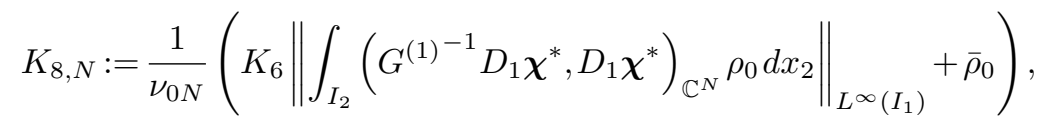


and

$$
\chi^{*}=\left(\chi_{1}^{*}, \ldots, \chi_{N}^{*}\right)^{T},
$$

provided that the regularity conditions (3.32) and $D_{1} \chi_{\ell} \in \tilde{L}^{2, \infty}(\Omega)$ for $1 \leq \ell \leq N$ are valid.

REMARK 3.18. Property (3.31) implies that

$$
K_{8, N} \leq \frac{1}{\nu_{0 N}}\left(\frac{K_{6}}{\nu_{N}^{(1)}}\left\|\sqrt{\rho}_{0}\left|D_{1} \chi\right|\right\|_{\tilde{L}^{2, \infty}(\Omega)}^{2}+\bar{\rho}_{0}\right) .
$$

Proof. Let $\mathbf{c} \in\left[H_{0}^{1}(\Omega)\right]^{N}$ and $v \in \mathcal{S}_{N}$ be related by the first expansion (3.10). Inequality (3.23) and bound (3.5) imply (3.35) with $K_{7, N}=K_{3, N}^{2}$.

On the other hand, we can write down the inequality

$$
\int_{\Omega}|D v|^{2} d x \geq \frac{1}{\bar{\rho}_{0}} \int_{\Omega}\left|D_{1} v\right|^{2} \rho_{0} d x+\frac{1}{K_{6}} \int_{\Omega}\left(\kappa_{0}\left|D_{2} v\right|^{2}+V_{0}|v|^{2}\right) d x,
$$

see the second intermediate inequality in (3.34). Using the equality $D_{1}\left(c_{\ell} \chi_{\ell}\right)=D_{1} c_{\ell}$. $\chi_{\ell}+c_{\ell} D_{1} \chi_{\ell}$ and the elementary inequality

$$
|a+b|^{2} \geq(1-\gamma)|a|^{2}-\left(\frac{1}{\gamma}-1\right)|b|^{2} \text { for any } a, b \in \mathbb{C}, \gamma>0,
$$

we have

$$
\int_{\Omega}\left|D_{1} v\right|^{2} \rho_{0} d x \geq(1-\gamma) \int_{\Omega}\left|\sum_{\ell=1}^{N} D_{1} c_{\ell} \cdot \chi_{\ell}\right|^{2} \rho_{0} d x-\left(\frac{1}{\gamma}-1\right) \int_{\Omega}\left|\sum_{\ell=1}^{N} c_{\ell} D_{1} \chi_{\ell}\right|^{2} \rho_{0} d x .
$$

Applying identity (3.21) and the equality in (3.34), for $0<\gamma<1$, we obtain

$$
\begin{aligned}
\int_{\Omega}|D v|^{2} d x \geq & \frac{1-\gamma}{\bar{\rho}_{0}} \int_{I_{1}}\left(G^{(0)} D_{1} \mathbf{c}, D_{1} \mathbf{c}\right)_{\mathbb{C}^{N}} d x_{1} \\
& -\left(\frac{1}{\gamma}-1\right) \frac{1}{\bar{\rho}_{0}} \int_{\Omega}\left|\left(\mathbf{c}, D_{1} \chi^{*}\right)_{\mathbb{C}^{N}}\right|^{2} \rho_{0} d x+\frac{1}{K_{6}} \int_{I_{1}}\left(G^{(1)} \mathbf{c}, \mathbf{c}\right)_{\mathbb{C}^{N}} d x_{1} .
\end{aligned}
$$

Furthermore, the following inequalities hold:

$$
\begin{aligned}
& \int_{\Omega}\left|\left(\mathbf{c}, D_{1} \chi^{*}\right)_{\mathbb{C}^{N}}\right|^{2} \rho_{0} d x \\
\leq & \int_{\Omega}\left(G^{(1)} \mathbf{c}, \mathbf{c}\right)_{\mathbb{C}^{N}} \cdot\left(G^{(1)^{-1}} D_{1} \chi^{*}, D_{1} \chi^{*}\right)_{\mathbb{C}^{N}} \rho_{0} d x \\
\leq & \operatorname{ess} \sup _{I_{1}} \int_{I_{2}}\left(G^{(1)^{-1}} D_{1} \chi^{*}, D_{1} \chi^{*}\right)_{\mathbb{C}^{N}} \rho_{0} d x_{2} \cdot \int_{I_{1}}\left(G^{(1)} \mathbf{c}, \mathbf{c}\right)_{\mathbb{C}^{N}} d x_{1} .
\end{aligned}
$$

Exploiting property (3.3) and taking $\gamma$ such that

$$
\left(\frac{1}{\gamma}-1\right) \frac{1}{\bar{\rho}_{0}}\left\|\int_{I_{2}}\left(G^{(1)^{-1}} D_{1} \chi^{*}, D_{1} \chi^{*}\right)_{\mathbb{C}^{N}} \rho_{0} d x_{2}\right\|_{L^{\infty}\left(I_{1}\right)}=\frac{1}{K_{6}},
$$


from (3.36) we obtain (3.35) with $K_{7, N}=K_{8, N}$.

Note that although property (3.35) for $K_{7, N}=K_{8, N}$ may appear more cumbersome, it can ensure weaker dependence on $N$ (or even independence from $N$ ); see section 4 below.

Proposition 3.19. For any $\mathbf{f}^{(N)} \in\left[H^{-1}\left(I_{1}\right)\right]^{N}$, there exists a unique weak solution $\mathbf{c}^{(N)} \in\left[H_{0}^{1}\left(I_{1}\right)\right]^{N}$ of the Galerkin BVP problem (3.24), (3.25), which satisfies the bound

$$
\left\|\mathbf{c}^{(N)}\right\|_{\left[H_{0}^{1}\left(I_{1}\right)\right]^{N}} \leq \nu^{-1} K_{7, N}\left\|\mathbf{f}^{(N)}\right\|_{\left[H^{-1}\left(I_{1}\right)\right]^{N}} .
$$

Under the regularity conditions (2.8) and (3.16), for any $\mathbf{f}^{(N)} \in\left[L^{2}\left(I_{1}\right)\right]^{N}, \mathbf{c}^{(N)}$ is in fact a strong solution which belongs to $\left[H^{2} \cap H_{0}^{1}\left(I_{1}\right)\right]^{N}$ and satisfies the system of ODEs (3.24) (or (3.28)) in $\left[L^{2}\left(I_{1}\right)\right]^{N}$. Moreover, the following bound holds:

$$
\left\|\mathbf{c}^{(N)}\right\|_{\left[H^{2}\left(I_{1}\right)\right]^{N}} \leq K_{9, N}\left\|\mathbf{f}^{(N)}\right\|_{\left[L^{2}\left(I_{1}\right)\right]^{N}}
$$

(an expression for $K_{9, N}$ is omitted).

Proof. Corollary 3.8, Proposition 3.17, and the Riesz-Fischer representation theorem imply the first part of the proposition.

Next, under conditions (2.8) and (3.16) and for $\mathbf{f}^{(N)} \in\left[L^{2}\left(I_{1}\right)\right]^{N}$, we first obtain from (3.26) that $A D_{1} \mathbf{c}^{(N)}$ has a derivative $D_{1}\left(A D_{1} \mathbf{c}^{(N)}\right) \in\left[L^{1}\left(I_{1}\right)\right]^{N}$. Equation (3.28) holds in $\left[L^{1}\left(I_{1}\right)\right]^{N}$ as well and the bound

$$
\begin{aligned}
\left\|\left|D_{1}\left(A D_{1} \mathbf{c}^{(N)}\right)\right|\right\|_{L^{q}\left(I_{1}\right)} \leq 2\left\|\left|A^{(0)}\right|\right\|_{I_{1}}\left\|\left|D_{1} \mathbf{c}^{(N)}\right|\right\|_{L^{\frac{2 q}{2-q}\left(I_{1}\right)}} \\
+\left\|\left|B-D_{1} A^{(0)}\right|\right\|_{L^{q}\left(I_{1}\right)}\left\|\mathbf{c}^{(N)}\left|\left\|_{L^{\infty}\left(I_{1}\right)}+\right\|\right| \mathbf{f}^{(N)} \mid\right\|_{L^{q}\left(I_{1}\right)}
\end{aligned}
$$

is valid for $q=1$.

Consequently $\left|D_{1} \mathbf{c}^{(N)}\right| \in L^{\infty}\left(I_{1}\right)$ and there exists the derivative

$$
D_{1}^{2} \mathbf{c}^{(N)}=A^{-1}\left[D_{1}\left(A D_{1} \mathbf{c}^{(N)}\right)-D_{1} A \cdot D_{1} \mathbf{c}^{(N)}\right] \in\left[L^{2}\left(I_{1}\right)\right]^{N},
$$

by taking into account that bound (3.39) is now valid for $q=2$ as well, $\left|D_{1} A\right| \in L^{2}\left(I_{1}\right)$ (see bound (3.17)), and $A^{-1} \in\left[C\left(\bar{I}_{1}\right)\right]^{N \times N}$ (see also inequalities (3.18)). This implies bound (3.38). A more general result can be found in [24].

We do not intend to go deeply into the problem of error estimation for the method and confine ourselves by the following standard result (for example see [1, 23]).

Proposition 3.20. The following optimal $\mathcal{E}(\Omega)$-error equality holds:

$$
\left\|u-v^{(N)}\right\|_{\mathcal{E}(\Omega)}=\min _{\varphi \in S_{N}}\|u-\varphi\|_{\mathcal{E}(\Omega)},
$$

together with the $L^{2}(\Omega)$-error bound

$$
\left\|u-v^{(N)}\right\|_{L^{2}(\Omega)} \leq \sup _{w \in H_{0}^{1}(\Omega): \mathcal{H} w \in L^{2}(\Omega)}\left[\frac{\min _{\varphi \in S_{N}}\|w-\varphi\|_{\mathcal{E}(\Omega)}}{\|\mathcal{H} w\|_{L^{2}(\Omega)}}\right] \min _{\varphi \in S_{N}}\|u-\varphi\|_{\mathcal{E}(\Omega)} .
$$


Proof. We recall that equality (3.40) (even for the infinite-dimensional closed $S_{N}$ ) follows from the identity

$$
\mathcal{L}_{\Omega}\left(u-v^{(N)}, u-v^{(N)}\right)=\mathcal{L}_{\Omega}\left(u-v^{(N)}, u-\varphi\right) \text { for all } \varphi \in S_{N}
$$

(see the integral identities (2.4) and (2.17)) and that the $L^{2}(\Omega)$-error bound (3.41) follows from the related identity

$$
\left\|u-v^{(N)}\right\|_{L^{2}(\Omega)}^{2}=\mathcal{L}_{\Omega}\left(u-v^{(N)}, w\right)=\mathcal{L}_{\Omega}\left(u-v^{(N)}, w-\varphi\right) \text { for all } \varphi \in S_{N},
$$

where $w \in H_{0}^{1}(\Omega)$ is the solution of the auxiliary problem $\mathcal{H} w=u-v^{(N)}$.

Remark 3.21. The Galerkin method described above may be extended to solve an elliptic BVP in an $n$-dimensional cylindrical domain $\Omega=G \times I_{n}$, where $G$ is an $(n-1)$ dimensional bounded domain, $n>2$ and $I_{n}:=(0, X)$. This is of increasing physical interest now. The approximate solution (2.11) may be considered once more with $\hat{x}_{n}=\left(x_{1}, \ldots, x_{n-1}\right)$ replacing $x_{1}$ and $x_{n}$ replacing $x_{2}$. For $c_{\ell} \in H_{0}^{1}(G)$ and $\chi_{\ell} \in L^{\infty, 2}(\Omega)$, $D \chi_{\ell} \in\left[L^{q_{n}, 2}(\Omega)\right]^{n}$, with $q_{n}>2$ for $n=3$ and $q_{n}=n-1$ for $n>3,\left.\chi_{\ell}\right|_{x_{n}=0, X}=0,1 \leq$ $\ell \leq N$, we obtain that $v^{(N)} \in H_{0}^{1}(\Omega)$. In the case of the $n$-dimensional version of the self-adjoint elliptic BVP (2.1), (2.2) (with $n$ replacing 2 as upper limit in the sum), the Galerkin BVP for the vector-function $\mathbf{c}^{(N)}$ of the coefficients of $v^{(N)}$ has the following form:

$$
\begin{aligned}
& \mathcal{H}_{N} \mathbf{c}^{(N)}:=-\sum_{i=1}^{n-1} D_{i}\left(\sum_{j=1}^{n-1} A^{(i j)} D_{j} \mathbf{c}^{(N)}+A^{(i 0)} \mathbf{c}^{(N)}\right) \\
&+\sum_{i=1}^{n-1} A^{(i 0)^{*}} D_{i} \mathbf{c}^{(N)}+B \mathbf{c}^{(N)}=\mathbf{f}^{(N)} \text { on } G, \\
&\left.\mathbf{c}^{(N)}\right|_{\partial G}=0,
\end{aligned}
$$

where the $N \times N$ matrices $A^{(i j)}, A^{(i 0)}$ and $B$ have entries, for any $1 \leq i \leq n-1,1 \leq$ $j \leq n-1,1 \leq k \leq N, 1 \leq \ell \leq N$, and a.a. $\hat{x}_{n} \in G$

$$
\begin{aligned}
& a_{k \ell}^{(i j)}\left(\hat{x}_{n}\right)=\int_{I_{n}}\left(\kappa_{i j} \chi_{k}^{*} \chi_{\ell}\right)\left(\hat{x}_{n}, x_{n}\right) d x_{n}, \\
& a_{k \ell}^{(i 0)}\left(\hat{x}_{n}\right)=\int_{I_{n}}\left(\chi_{k}^{*} \sum_{j=1}^{n} \kappa_{i j} D_{j} \chi_{\ell}\right)\left(\hat{x}_{n}, x_{n}\right) d x_{n}, \\
& b_{k \ell}^{(0)}\left(\hat{x}_{n}\right)=\int_{I_{n}}\left(\sum_{i, j=1}^{n} \kappa_{i j} D_{j} \chi_{\ell} \cdot D_{i} \chi_{k}^{*}+V \chi_{k}^{*} \chi_{\ell}\right)\left(\hat{x}_{n}, x_{n}\right) d x_{n},
\end{aligned}
$$

and $\mathbf{f}^{(N)}$ is a vector having the components $f_{k} \in H^{-1}(G), 1 \leq k \leq N$, such that

$$
\left\langle f_{k}, \eta\right\rangle_{G}=\left\langle f, \eta \chi_{k}\right\rangle_{\Omega} \text { for any } \eta \in H_{0}^{1}(G)
$$

compare with (3.12)-(3.14) and (3.27). Equation (3.42) can be classified as a self- 
adjoint strongly elliptic system of PDEs since $A^{(i j)}=A^{(j i)}=A^{(i j)^{*}}, B=B^{*}$ and

$$
\begin{aligned}
& \sum_{i, j=1}^{n-1}\left(A^{(i j)} \xi^{(j)}, \xi^{(i)}\right)_{\mathbb{C}^{N}}=\int_{I_{n}} \sum_{i, j=1}^{n-1} \kappa_{i j} v^{(j)} v^{(i)^{*}} d x_{n} \\
& \geq \nu \int_{I_{n}} \sum_{i=1}^{n-1}\left|v^{(i)}\right|^{2} d x_{n} \geq \frac{\nu \nu_{0 N}}{\bar{\rho}_{0}} \sum_{i=1}^{n-1}\left|\xi^{(i)}\right|^{2} \text { for any } \xi^{(1)}, \ldots, \xi^{(n-1)} \in \mathbb{C}^{N}
\end{aligned}
$$

compare with (3.18). Here $v^{(i)}:=\sum_{\ell=1}^{N} \xi_{\ell}^{(i)} \chi_{\ell}$, and $\nu_{0 N}>0$ is introduced quite similarly to (3.3) (see also (3.1) and (3.21)).

\section{A specific choice of the basis functions}

An important choice of the basis functions is related, for physical reasons, to a $1 \mathrm{D}$ eigenvalue problem with respect to $x_{2}$ depending parametrically on $x_{1} \in I_{1}$

$$
\begin{aligned}
& \mathcal{H}_{0} \chi:=-D_{2}\left(\kappa_{0} D_{2} \chi\right)+V_{0} \chi=\alpha \rho_{0} \chi \text { in } I_{2}, \\
& \left.\chi\right|_{x_{2}=0, X_{2}}=0
\end{aligned}
$$

where the coefficients $\kappa_{0}, V_{0}$ and $\rho_{0}$ depend on $x_{1}$ and $x_{2}$ and satisfy the above conditions (3.29). Recall that in its general (i.e. weak) formulation, this problem consists of finding the eigenfunctions $\chi\left(x_{1}, \cdot\right) \in H_{0}^{1}\left(I_{2}\right), \chi\left(x_{1}, \cdot\right) \not \equiv 0$ and the corresponding eigenvalues $\alpha\left(x_{1}\right)$ satisfying the integral identity

$$
\begin{aligned}
\mathcal{L}_{0 I_{2}}\left[x_{1}\right]\left(\chi\left(x_{1}, \cdot\right), \zeta(\cdot)\right) & :=\int_{I_{2}}\left[\left(\kappa_{0} D_{2} \chi\right)\left(x_{1}, x_{2}\right)\left(D_{2} \zeta^{*}\right)\left(x_{2}\right)+\left(V_{0} \chi\right)\left(x_{1}, x_{2}\right) \zeta^{*}\left(x_{2}\right)\right] d x_{2} \\
& =\alpha\left(x_{1}\right) \int_{I_{2}}\left(\rho_{0} \chi\right)\left(x_{1}, x_{2}\right) \zeta^{*}\left(x_{2}\right) d x_{2} \text { for any } \zeta \in H_{0}^{1}\left(I_{2}\right) .
\end{aligned}
$$

Below, we mainly omit $\left[x_{1}\right]$ in the notations for brevity.

According to well known results, for a.a. $x_{1} \in I_{1}$ the problem has a sequence of real eigenvalues such that

$$
0<\alpha_{1}\left(x_{1}\right)<\cdots<\alpha_{\ell}\left(x_{1}\right)<\ldots, \quad \alpha_{\ell}\left(x_{1}\right) \rightarrow \infty \text { as } \ell \rightarrow \infty
$$

and the corresponding real eigenfunctions $\left\{\chi_{\ell}\left(x_{1}, \cdot\right)\right\}_{\ell=1}^{\infty}$ are orthogonal in $L^{2}\left(I_{2}\right)$ with the weight $\rho_{0}\left(x_{1}, \cdot\right)$ :

$$
\int_{I_{2}}\left(\chi_{k} \chi \ell \rho_{0}\right)\left(x_{1}, x_{2}\right) d x_{2}=0 \text { for any } k \neq \ell .
$$

Moreover, we assume that they are normalized:

$$
\int_{I_{2}}\left(\chi_{k}^{2} \rho_{0}\right)\left(x_{1}, x_{2}\right) d x_{2}=1 \text { for any } k .
$$

From (4.3)-(4.5) one deduces that these eigenfunctions have a second orthogonality property

$$
\int_{I_{2}}\left(\kappa_{0} D_{2} \chi_{k} \cdot D_{2} \chi_{\ell}+V_{0} \chi_{k} \chi_{\ell}\right)\left(x_{1}, x_{2}\right) d x_{2}=\alpha_{k}\left(x_{1}\right) \delta_{k l} \text { for any } k, \ell,
$$

with $\delta_{k k}=1$ and $\delta_{k \ell}=0$ for $k \neq \ell$. 
Furthermore, for any $w \in L^{2}(\Omega)$ and for a.a. $x_{1} \in I_{1}$ the Fourier expansion

$$
w\left(x_{1}, x_{2}\right)=\sum_{\ell=1}^{\infty} \widetilde{w}_{\ell}\left(x_{1}\right) \chi_{\ell}\left(x_{1}, x_{2}\right), \text { with } \widetilde{w}_{\ell}\left(x_{1}\right):=\int_{I_{2}}\left(w \chi_{\ell} \rho_{0}\right)\left(x_{1}, x_{2}\right) d x_{2}
$$

converges in $L^{2}\left(I_{2}\right)$, and the Parseval equality holds:

$$
\int_{I_{2}}\left(|w|^{2} \rho_{0}\right)\left(x_{1}, x_{2}\right) d x_{2}=\sum_{\ell=1}^{\infty}\left|\widetilde{w}_{\ell}\right|^{2}\left(x_{1}\right) .
$$

Moreover, if in addition $D_{2} w \in L^{2}(\Omega)$ and $\left.w\right|_{x_{2}=0, X_{2}}=0$, then the expansion converges in $H_{0}^{1}\left(I_{2}\right)$, and the second Parseval equality holds:

$$
\int_{I_{2}}\left[\kappa_{0}\left|D_{2} w\right|^{2}+V_{0}|w|^{2}\right]\left(x_{1}, x_{2}\right) d x_{2}=\sum_{\ell=1}^{\infty} \alpha_{\ell}\left(x_{1}\right)\left|\widetilde{w}_{\ell}\right|^{2}\left(x_{1}\right) .
$$

We also recall the Courant-Fischer minmax principle, for $\ell \geq 1$,

$$
\alpha_{\ell}\left(x_{1}\right)=\min _{L_{\ell} \subset H_{0}^{1}\left(I_{2}\right)} \max _{\zeta \in L_{\ell}} \mathcal{R}_{I_{2}}\left(x_{1} ; \zeta\right), \text { with } \mathcal{R}_{I_{2}}\left(x_{1} ; \zeta\right):=\frac{\mathcal{L}_{0 I_{2}}\left[x_{1}\right](\zeta, \zeta)}{\int_{I_{2}}\left(\zeta^{2} \rho_{0}\right)\left(x_{1}, x_{2}\right) d x_{2}},
$$

where $\mathcal{R}_{I_{2}}\left(x_{1} ; \zeta\right)$ is the Rayleigh quotient. The minimum in (4.10) is taken over all the $\ell$-dimensional subspaces $L_{\ell}$ in real $H_{0}^{1}\left(I_{2}\right)$, and the case $\zeta \equiv 0$ is automatically excluded.

Consequently, for any $\ell \geq 1$, we have that $\alpha_{\ell} \in L^{\infty}\left(I_{1}\right)$ and the following two-sided bound holds:

$$
K_{10}^{-1} \ell^{2} \leq \alpha_{\ell}\left(x_{1}\right) \leq K_{11} \ell^{2} \text { for a.a. } x_{1} \in I_{1}
$$

with

$$
K_{10}^{-1}=\frac{\underline{\kappa}_{0}}{\bar{\rho}_{0}}\left(\frac{\pi}{X_{2}}\right)^{2}, \quad K_{11}=\frac{K_{6}}{\underline{\rho}_{0}}\left(\frac{\pi}{X_{2}}\right)^{2} .
$$

For such a choice of $\left\{\chi_{\ell}\right\}_{\ell=1}^{\infty}$, by virtue of properties (4.4)-(4.6) we obtain

$$
G_{0}=\mathbb{I}, \quad G^{(1)}=\operatorname{diag}\left\{\alpha_{1}, \ldots, \alpha_{N}\right\},
$$

and thus $\nu_{0 N}=1$ and $\nu_{N}^{(1)}=\operatorname{ess} \inf _{I_{1}} \alpha_{1} \geq K_{10}^{-1}$ are independent of $N$. In particular, if $\rho_{0}=\kappa_{11}$, then $A=\mathbb{I}$ as well and the Galerkin system of ODEs (3.24) becomes simpler.

Note that according to the integral identity (4.3), for a.a. $x_{1} \in I_{1}$, the product $\left(\kappa_{0} D_{2} \chi_{\ell}\right)\left(x_{1}, \cdot\right)$ has the derivative

$$
D_{2}\left(\kappa_{0} D_{2} \chi_{\ell}\right)\left(x_{1}, \cdot\right)=\left(V_{0} \chi_{\ell}-\alpha_{\ell} \chi_{\ell}\right)\left(x_{1}, \cdot\right) \in L^{1}\left(I_{2}\right) .
$$

Thus $\left(\kappa_{0} D_{2} \chi_{\ell}\right)\left(x_{1}, \cdot\right) \in C\left(\bar{I}_{2}\right)$ and $D_{2} \chi_{\ell}\left(x_{1}, \cdot\right) \in L^{\infty}\left(I_{2}\right)$. Moreover, if $\kappa_{0}\left(x_{1}, \cdot\right) \in C\left(\bar{I}_{2}\right)$, then $\chi_{\ell}\left(x_{1}, \cdot\right) \in C^{1}\left(\bar{I}_{2}\right)$.

Each function $\chi_{\ell}\left(x_{1}, \cdot\right)$ is unique up to the factor \pm 1 . We choose $\chi_{\ell}\left(x_{1}, \cdot\right)$ by specifying either that $\left(\kappa_{0} D_{2} \chi_{\ell}\right)\left(x_{1}, 0\right)>0$ (the equality $\left(\kappa_{0} D_{2} \chi_{\ell}\right)\left(x_{1}, 0\right)=0$ cannot hold) or, when $\kappa_{0}\left(x_{1}, \cdot\right) \in C\left(\bar{I}_{2}\right)$, simply that $\left(D_{2} \chi_{\ell}\right)\left(x_{1}, 0\right)>0$. Then it is possible to derive that $\chi_{\ell} \in H^{1}(\Omega)$ under some additional conditions on $\kappa_{0}, V_{0}$ and $\rho_{0}$ (the rather lengthy proof is omitted). 
REMARK 4.1. Optimally choosing the functions $\kappa_{0}, \rho_{0}$, and $V_{0}$ is a practically important question. We will not touch upon this topic in the paper and only mention that some physical arguments are invoked to solve this question, which would deserve some mathematical work to be rigorously presented. One may keep in mind the simple choice $\kappa_{0}:=\kappa_{22}, V_{0}:=V$ and $\rho_{0}:=1$ (or alternatively $\rho_{0}:=\kappa_{11}$ ).

Also recall that formally one of the coefficients $\kappa_{0}$ and $\rho_{0}$ can be reduced to 1 . For example, one can normalize equation (4.1) by multiplying it by

$$
\left[\frac{1}{\kappa_{0}}\right]_{I_{2}}\left(x_{1}\right):=\frac{1}{X_{2}} \int_{I_{2}} \frac{d x_{2}}{\kappa_{0}\left(x_{1}, x_{2}\right)}
$$

(in order to keep the domain $\Omega$ the same) and then change the variable $y_{2}:=$

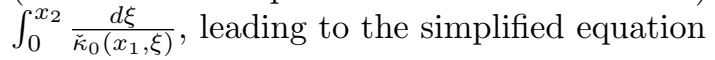

$$
-D_{y_{2}}^{2} \check{\chi}+\check{V_{0}} \check{\chi}=\alpha \check{\rho_{0}} \check{\chi} \text { in } I_{2},
$$

where $\chi\left(x_{1}, x_{2}\right)=\check{\chi}\left(x_{1}, y_{2}\left(x_{1}, x_{2}\right)\right)$ and

$$
\check{\kappa}_{0}:=\left[\frac{1}{\kappa_{0}}\right]_{I_{2}} \kappa_{0}, \quad \check{V_{0}}:=\left[\frac{1}{\kappa_{0}}\right]_{I_{2}} \check{\kappa}_{0} V_{0}, \quad \check{\rho_{0}}:=\left[\frac{1}{\kappa_{0}}\right]_{I_{2}} \check{\kappa}_{0} \rho_{0} .
$$

But we have take into account then that $D_{1} \chi=D_{1} \check{\chi}+D_{1} y_{2} \cdot D_{y_{2}} \check{\chi}$.

Now we turn to a study of approximation properties of the space $S_{N}$ spanned by the first $N$ eigenfunctions of the auxiliary eigenvalue problem (4.1), (4.2). In the spirit of [1], we introduce a family of associated Hilbert spaces $\mathbf{H}^{0, \theta}(\Omega), \theta \geq$ 0 , consisting of functions $w \in L^{2}(\Omega)$ such that the series $\sum_{\ell=1}^{\infty} \alpha_{\ell}^{\theta / 2} \tilde{w}_{\ell} \chi_{\ell}$ converges in $L^{2}(\Omega)$, equipped with the norm $\|w\|_{\mathbf{H}^{0, \theta}(\Omega)}=\left[\int_{I_{1}} \sum_{\ell=1}^{\infty} \alpha_{\ell}^{\theta}\left|\tilde{w}_{\ell}\right|^{2} d x_{1}\right]^{1 / 2}$. Clearly $\mathbf{H}^{0, \theta_{1}}(\Omega) \subset \mathbf{H}^{0, \theta}(\Omega)$ and $\mathbf{H}^{0, \theta_{1}}(\Omega) \not \equiv \mathbf{H}^{0, \theta}(\Omega)$ for any $0 \leq \theta<\theta_{1}$.

It follows from the Parseval equalities (4.8) and (4.9) that

$$
\|w\|_{\mathbf{H}^{0,0}(\Omega)}=\left\|\sqrt{\rho_{0}} w\right\|_{L^{2}(\Omega)}, \quad\|w\|_{\mathbf{H}^{0,1}(\Omega)}=\left[\int_{\Omega}\left[\kappa_{0}\left|D_{2} w\right|^{2}+V_{0}|w|^{2}\right] d x\right]^{1 / 2} ;
$$

thus

$$
\mathbf{H}^{0,0}(\Omega)=L^{2}(\Omega) \text { and } \mathbf{H}^{0,1}(\Omega):=\left\{w \in L^{2}(\Omega) ; D_{2} w \in L^{2}(\Omega),\left.w\right|_{x_{2}=0, X_{2}}=0\right\},
$$

up to an equivalence of norms.

Next, the space $\mathbf{H}^{0,2}(\Omega)$ consists of functions $w \in \mathbf{H}^{0,1}(\Omega)$ having a derivative $D_{2}\left(\kappa_{0} D_{2} w\right) \in L^{1}(\Omega)$ and such that $\mathcal{H}_{0} w \in L^{2}(\Omega)$, with the norm $\|w\|_{\mathbf{H}^{0,2}(\Omega)}=$ $\left\|\frac{1}{\sqrt{\rho_{0}}} \mathcal{H}_{0} w\right\|_{L^{2}(\Omega)}$. The last two conditions on $w$ are reduced to the simple condition $D_{2}^{2} w \in L^{2}(\Omega)$ provided that $D_{2} \kappa_{0}, V_{0} \in \widetilde{L}^{2, \infty}(\Omega)$. Moreover, $\mathbf{H}^{0, \theta}(\Omega)$ for $\theta>2$ is the space of functions $w \in \mathbf{H}^{0,2}(\Omega)$ such that $\rho_{0}^{-1} \mathcal{H}_{0} w \in \mathbf{H}^{0, \theta-2}(\Omega)$ and

$$
\|w\|_{\mathbf{H}^{0, \theta}(\Omega)}=\left\|\rho_{0}^{-1} \mathcal{H}_{0} w\right\|_{\mathbf{H}^{0, \theta-2}(\Omega)} .
$$

We define a projector $\mathcal{P}_{N}: L^{2}(\Omega) \rightarrow S_{N}^{0}$ by the formula

$$
\left(\mathcal{P}_{N} w\right)\left(x_{1}, x_{2}\right):=\sum_{\ell=1}^{N} \widetilde{w}_{\ell}\left(x_{1}\right) \chi_{\ell}\left(x_{1}, x_{2}\right) \text { in } \Omega, \text { for } w \in L^{2}(\Omega),
$$


where the coefficients $\widetilde{w}_{\ell}$ are given by formula (4.7).

We also define the auxiliary energy norm

$$
\|w\|_{\mathcal{E}_{0}(\Omega)}:=\left[\int_{\Omega}\left(\rho_{0}\left|D_{1} w\right|^{2}+\kappa_{0}\left|D_{2} w\right|^{2}+V_{0}|w|^{2}\right) d x\right]^{1 / 2},
$$

which clearly coincides with $\|w\|_{\mathcal{E}(\Omega)}$ in the particular case $\varkappa=\operatorname{diag}\left\{\rho_{0}, \kappa_{0}\right\}$ and $V=$ $V_{0}$. Let $\underline{\alpha}_{\ell}:=\operatorname{ess} \inf _{I_{1}} \alpha_{\ell}$.

Proposition 4.2. Let $w \in \mathbf{H}^{0, \theta}(\Omega)$, for some $\theta \geq 0$. Then the $L^{2}(\Omega)$-approximation bound holds:

$$
\left\|\sqrt{\rho_{0}}\left(w-\mathcal{P}_{N} w\right)\right\|_{L^{2}(\Omega)} \leq \underline{\alpha}_{N+1}^{-\theta / 2}\|w\|_{\mathbf{H}^{0, \theta}(\Omega)} .
$$

If in addition $\theta \geq 1$ and $D_{1} w \in \mathbf{H}^{0, \theta-1}(\Omega)$, the $\mathcal{E}_{0}(\Omega)$-approximation bound holds:

$$
\left\|w-\mathcal{P}_{N} w\right\|_{\mathcal{E}_{0}(\Omega)} \leq \underline{\alpha}_{N+1}^{-(\theta-1) / 2}\left(\|w\|_{\mathbf{H}^{0, \theta}(\Omega)}+\left\|D_{1} w\right\|_{\mathbf{H}^{0, \theta-1}(\Omega)}\right)+\left\|\sqrt{\rho_{0}} \mathcal{R}_{N} w\right\|_{L^{2}(\Omega)},
$$

where $\mathcal{R}_{N} w:=D_{1} \mathcal{P}_{N} w-\mathcal{P}_{N} D_{1} w$.

Proof. Due to the first Parseval equality (4.8), we obtain

$$
\begin{aligned}
\left\|\sqrt{\rho_{0}}\left(w-\mathcal{P}_{N} w\right)\right\|_{L^{2}(\Omega)}^{2} & =\int_{I_{1}} \int_{I_{2}} \rho_{0}\left|w-\mathcal{P}_{N} w\right|^{2} d x_{2} d x_{1} \\
& =\int_{I_{1}} \sum_{\ell=N+1}^{\infty}\left|\widetilde{w}_{\ell}\right|^{2} d x_{1} \leq \underline{\alpha}_{N+1}^{-\theta}\|w\|_{\mathbf{H}^{0, \theta}(\Omega)}^{2} .
\end{aligned}
$$

Moreover, we also obtain

$$
\begin{aligned}
\left\|w-\mathcal{P}_{N} w\right\|_{\mathcal{E}_{0}(\Omega) \leq} & \left\|\sqrt{\rho_{0}}\left(D_{1} w-\mathcal{P}_{N} D_{1} w\right)\right\|_{\Omega} \\
& +\left\|\sqrt{\rho_{0}}\left(D_{1} \mathcal{P}_{N} w-\mathcal{P}_{N} D_{1} w\right)\right\|_{\Omega} \\
& +\left[\int_{I_{1}} \mathcal{L}_{0 I_{2}}\left(w-\mathcal{P}_{N} w, w-\mathcal{P}_{N} w\right) d x_{1}\right]^{1 / 2} .
\end{aligned}
$$

Due to the second Parseval equality (4.9), we have

$$
\mathcal{L}_{0 I_{2}}\left(w-\mathcal{P}_{N} w, w-\mathcal{P}_{N} w\right)=\sum_{\ell=N+1}^{\infty} \alpha_{\ell}\left|\widetilde{w}_{\ell}\right|^{2} .
$$

Then the second approximation bound (4.15) follows from the first one (4.14) with $D_{1} w$ replacing $w$ and estimating (4.17) similarly to (4.16).

A delicate matter is now to bound $\mathcal{R}_{N} w$. We first get a representation for $\mathcal{R}_{N} w$. Let $\dot{\chi}_{\ell}:=D_{1} \chi_{\ell}$ and $\dot{\chi}_{\ell, k}:=\widetilde{\left(\dot{\chi}_{\ell}\right)_{k}}$, for brevity.

Lemma 4.3. Let $w \in H^{1,0}(\Omega)$. The following formula holds

$$
\mathcal{R}_{N} w=\sum_{\ell=1}^{N} \widetilde{w}_{\ell}\left(\dot{\chi}_{\ell}-\mathcal{P}_{N} \dot{\chi}_{\ell}\right)-\sum_{\ell=N+1}^{\infty} \widetilde{w}_{\ell} \mathcal{P}_{N} \dot{\chi}_{\ell}
$$

where the series converges in $L^{2}(\Omega)$ (note that the representation does not contain $\left.D_{1} w\right)$. 
Proof. By differentiating in $x_{1}$ the formulas for $\mathcal{P}_{N} w$ and $\widetilde{w}_{\ell}$ as well as the orthonormality conditions (4.4) and (4.5), we derive

$$
\begin{aligned}
D_{1} \mathcal{P}_{N} w & =\sum_{\ell=1}^{N}\left(D_{1} \widetilde{w}_{\ell} \cdot \chi_{\ell}+\widetilde{w}_{\ell} \dot{\chi}_{\ell}\right) \quad \text { on } \Omega, \\
D_{1} \widetilde{w}_{\ell} & =\left(\widetilde{D_{1} w}\right)_{\ell}+\int_{I_{2}} w D_{1}\left(\chi_{\ell} \rho_{0}\right) d x_{2} \text { on } I_{1}
\end{aligned}
$$

and

$$
\int_{I_{2}}\left[\dot{\chi}_{\ell} \chi_{k} \rho_{0}+\chi_{\ell} D_{1}\left(\chi_{k} \rho_{0}\right)\right] d x_{2}=0 \text { on } I_{1} \text {, for any } k, \ell \text {. }
$$

Expanding $w$ with respect to the system $\left\{\chi_{\ell}\right\}_{\ell=1}^{\infty}$ and applying the last formula, we further calculate

$$
\begin{aligned}
& \int_{I_{2}} w D_{1}\left(\chi_{k} \rho_{0}\right) d x_{2}=\int_{I_{2}} \sum_{\ell=1}^{\infty} \widetilde{w}_{\ell} \chi_{\ell} D_{1}\left(\chi_{k} \rho_{0}\right) d x_{2} \\
& =-\sum_{\ell=1}^{\infty} \widetilde{w}_{\ell} \int_{I_{2}} \dot{\chi}_{\ell} \chi_{k} \rho_{0} d x_{2}=-\sum_{\ell=1}^{\infty} \widetilde{w}_{\ell} \dot{\chi}_{\ell, k} \text { on } I_{1} .
\end{aligned}
$$

Gathering these formulas and rearranging the summands, we find

$$
\begin{aligned}
D_{1} \mathcal{P}_{N} w-\mathcal{P}_{N} D_{1} w & =\sum_{\ell=1}^{N} D_{1} \widetilde{w}_{\ell} \cdot \chi_{\ell}+\widetilde{w}_{\ell} \dot{\chi}_{\ell}-\widetilde{\left(D_{1} w_{\ell}\right)} \chi_{\ell} \\
& =\sum_{\ell=1}^{N} \widetilde{w}_{\ell} \dot{\chi}_{\ell}+\sum_{k=1}^{N} \int_{I_{2}} w D_{1}\left(\chi_{k} \rho_{0}\right) d x_{2} \cdot \chi_{k} \\
& =\sum_{\ell=1}^{N} \widetilde{w}_{\ell} \dot{\chi}_{\ell}-\sum_{k=1}^{N} \sum_{\ell=1}^{\infty} \widetilde{w}_{\ell} \dot{\chi}_{\ell, k} \chi_{k} \\
& =\sum_{\ell=1}^{N} \widetilde{w}_{\ell} \dot{\chi}_{\ell}-\sum_{\ell=1}^{\infty} \widetilde{w}_{\ell}\left(\sum_{k=1}^{N} \dot{\chi}_{\ell, k} \chi_{k}\right) .
\end{aligned}
$$

This directly implies the result.

CoRollary 4.4. If $w \in H^{1,0}(\Omega) \cap \mathbf{H}^{0, \theta}(\Omega)$ for some $\theta \geq 0$, then the following bound holds:

$$
\left\|\sqrt{\rho_{0}} \mathcal{R}_{N} w\right\|_{L^{2}(\Omega)} \leq\left\|\sqrt{\left[s_{1 N}^{(\theta)}\right]^{2}+\left[s_{2 N}^{(\theta)}\right]^{2}}\right\|_{L^{\infty}(\Omega)}\|w\|_{\mathbf{H}^{0, \theta}(\Omega)}
$$

where

$$
\begin{aligned}
& s_{1 N}^{(\theta)}:=\left[\sum_{\ell=1}^{N} \alpha_{\ell}^{-\theta}\left\|\sqrt{\rho_{0}}\left(\dot{\chi}_{\ell}-\mathcal{P}_{N} \dot{\chi}_{\ell}\right)\right\|_{L^{2}\left(I_{2}\right)}^{2}\right]^{1 / 2}=\left[\sum_{\ell=1}^{N} \alpha_{\ell}^{-\theta} \sum_{k=N+1}^{\infty} \dot{\chi}_{\ell, k}^{2}\right]^{1 / 2}, \\
& s_{2 N}^{(\theta)}:=\left[\sum_{\ell=N+1}^{\infty} \alpha_{\ell}^{-\theta}\left\|\sqrt{\rho_{0}} \mathcal{P}_{N} \dot{\chi}_{\ell}\right\|_{L^{2}\left(I_{2}\right)}^{2}\right]^{1 / 2}=\left[\sum_{\ell=N+1}^{\infty} \alpha_{\ell}^{-\theta} \sum_{k=1}^{N} \dot{\chi}_{\ell, k}^{2}\right]^{1 / 2} .
\end{aligned}
$$


In contrast to other bounds in the paper, the case $\left\|\sqrt{\left[s_{1 N}^{(\theta)}\right]^{2}+\left[s_{2 N}^{(\theta)}\right]^{2}}\right\|_{L^{\infty}(\Omega)}=\infty$ is not excluded (in general).

Proof. Applying the Cauchy-Schwartz inequality, we have

$$
\begin{aligned}
\left\|\sqrt{\rho_{0}} \mathcal{R}_{N} w\right\|_{I_{2}} \leq & \sum_{\ell=1}^{N}\left|\widetilde{w}_{\ell}\right|\left\|\sqrt{\rho_{0}}\left(\dot{\chi}_{\ell}-\mathcal{P}_{N} \dot{\chi}_{\ell}\right)\right\|_{I_{2}}+\sum_{\ell=N+1}^{\infty}\left|\widetilde{w}_{\ell}\right|\left\|\sqrt{\rho_{0}} \mathcal{P}_{N} \dot{\chi}_{\ell}\right\|_{I_{2}} \\
\leq & \left(\sum_{\ell=1}^{N} \alpha_{\ell}^{\theta}\left|\widetilde{w}_{\ell}\right|^{2}\right)^{1 / 2}\left(\sum_{\ell=1}^{N} \alpha_{\ell}^{-\theta}\left\|\sqrt{\rho_{0}}\left(\dot{\chi}_{\ell}-\mathcal{P}_{N} \dot{\chi}_{\ell}\right)\right\|_{I_{2}}^{2}\right)^{1 / 2} \\
& +\left(\sum_{\ell=N+1}^{\infty} \alpha_{\ell}^{\theta}\left|\widetilde{w}_{\ell}\right|^{2}\right)^{1 / 2}\left(\sum_{\ell=N+1}^{\infty} \alpha_{\ell}^{-\theta}\left\|\sqrt{\rho_{0}} \mathcal{P}_{N} \dot{\chi}_{\ell}\right\|_{I_{2}}^{2}\right)^{1 / 2}
\end{aligned}
$$

Consequently

$$
\begin{aligned}
\left\|\sqrt{\rho_{0}} \mathcal{R}_{N} w\right\|_{\Omega} & \leq\left\|\left(\sum_{\ell=1}^{\infty} \alpha_{\ell}^{\theta}\left|\widetilde{w}_{\ell}\right|\right)^{1 / 2} \sqrt{\left[s_{1 N}^{(\theta)}\right]^{2}+\left[s_{2 N}^{(\theta)}\right]^{2}}\right\|_{I_{1}} \\
& \leq\|w\|_{\mathbf{H}^{0, \theta}(\Omega)}\left\|\sqrt{\left[s_{1 N}^{(\theta)}\right]^{2}+\left[s_{2 N}^{(\theta)}\right]^{2}}\right\|_{L^{\infty}\left(I_{1}\right)} .
\end{aligned}
$$

REMARK 4.5. The following formula holds

$$
\left\|\sqrt{\rho_{0}} \mathcal{R}_{N} w\right\|_{L^{2}(\Omega)}^{2}=\int_{I_{1}}\left\{\sum_{k=1}^{N}\left|\sum_{\ell=N+1}^{\infty} \widetilde{w}_{\ell} \dot{\chi}_{\ell, k}\right|^{2}+\sum_{k=N+1}^{\infty}\left|\sum_{\ell=1}^{N} \widetilde{w}_{\ell} \dot{\chi}_{\ell, k}\right|^{2}\right\} d x_{1},
$$

which is a consequence of representation (4.18) rewritten as

$$
\mathcal{R}_{N} w=\sum_{\ell=1}^{N} \widetilde{w}_{\ell} \sum_{k=N+1}^{\infty} \dot{\chi}_{\ell, k} \chi_{k}-\sum_{\ell=N+1}^{\infty} \widetilde{w}_{\ell} \sum_{k=1}^{N} \dot{\chi}_{\ell, k} \chi_{k} .
$$

Now we suppose that $\dot{\alpha}_{\ell}:=D_{1} \alpha_{\ell} \in L^{1}\left(I_{1}\right)$ and $\dot{\chi}_{\ell}\left(x_{1}, \cdot\right) \in H_{0}^{1}\left(I_{2}\right)$, for $\ell \geq 1$ and a.a. $x_{1} \in I_{1}$, and derive some their properties. Up to the end of this section, we also assume that

$$
\left\|D_{1} \kappa_{0}\right\|_{L^{\infty}(\Omega)}+\left\|D_{1} V_{0}\right\|_{\tilde{L}^{1, \infty}(\Omega)}+\left\|D_{1} \rho_{0}\right\|_{L^{\infty}(\Omega)} \leq \mu_{1,0}
$$

and set

$$
K_{12}:=\left\|\kappa_{0}^{(1)}\right\|_{L^{\infty}(\Omega)}+\left\|\frac{1}{\kappa_{0}}\right\|_{\tilde{L}^{1, \infty}(\Omega)}\left\|D_{1} V_{0}\right\|_{\tilde{L}^{1, \infty}(\Omega)}, \quad K_{13}:=\left\|\rho_{0}^{(1)}\right\|_{L^{\infty}(\Omega)},
$$

with $\kappa_{0}^{(1)}:=\left(D_{1} \kappa_{0}\right) / \kappa_{0}$ and $\rho_{0}^{(1)}:=\left(D_{1} \rho_{0}\right) / \rho_{0}$. 
Proposition 4.6.

1. The following simple formula holds, for $\ell \geq 1$ :

$$
\dot{\alpha}_{\ell}=\dot{\mathcal{L}}_{0 I_{2}}\left(\chi_{\ell}, \chi_{\ell}\right)-\alpha_{\ell} \int_{I_{2}} D_{1} \rho_{0} \cdot \chi_{\ell}^{2} d x_{2} \text { in } I_{1},
$$

where

$$
\begin{aligned}
& \dot{\mathcal{L}}_{0 I_{2}}\left[x_{1}\right]\left(\chi_{\ell}\left(x_{1}, \cdot\right), \zeta(\cdot)\right) \\
:= & \int_{I_{2}}\left[\left(D_{1} \kappa_{0} \cdot D_{2} \chi\right)\left(x_{1}, x_{2}\right)\left(D_{2} \zeta\right)\left(x_{2}\right)+\left(D_{1} V_{0} \cdot \chi\right)\left(x_{1}, x_{2}\right) \zeta\left(x_{2}\right)\right] d x_{2} .
\end{aligned}
$$

2. For $\ell \geq 1$ and a.a. $x_{1} \in I_{1}, \dot{\chi}_{\ell}\left(x_{1}, \cdot\right) \in H_{0}^{1}\left(I_{2}\right)$ is a weak solution of the degenerate $B V P$

$$
\begin{aligned}
& \mathcal{H}_{0} \dot{\chi}_{\ell}-\alpha_{\ell} \rho_{0} \dot{\chi}_{\ell}=F_{\ell}:=-\dot{\mathcal{H}}_{0} \chi_{\ell}+\left(\alpha_{\ell} D_{1} \rho_{0}+\dot{\alpha}_{\ell} \rho_{0}\right) \chi_{\ell} \text { in } I_{2}, \\
& \left.\dot{\chi}\right|_{x_{2}=0, X_{2}}=0,
\end{aligned}
$$

with $\dot{\mathcal{H}}_{0} \chi:=-D_{2}\left(D_{1} \kappa_{0} \cdot D_{2} \chi\right)+D_{1} V_{0} \cdot \chi$, i.e., $\dot{\chi}_{\ell}$ satisfies the integral identity

$$
\begin{aligned}
& \mathcal{L}_{0 I_{2}}\left(\dot{\chi}_{\ell}, \zeta\right)-\alpha_{\ell} \int_{I_{2}} \rho_{0} \dot{\chi}_{\ell} \zeta d x_{2}=\left\langle F_{\ell}, \zeta\right\rangle_{I_{2}}:=-\dot{\mathcal{L}}_{0 I_{2}}\left(\chi_{\ell}, \zeta\right) \\
& +\alpha_{\ell} \int_{I_{2}} D_{1} \rho_{0} \cdot \chi_{\ell} \zeta d x_{2}+\dot{\alpha}_{\ell} \int_{I_{2}} \rho_{0} \chi_{\ell} \zeta d x_{2} \text { for any real } \zeta \in H_{0}^{1}\left(I_{2}\right),
\end{aligned}
$$

where $F_{\ell} \in H^{-1}\left(I_{2}\right)$.

Moreover, the following formula holds

$$
\dot{\chi}_{\ell, \ell} \equiv \int_{I_{2}} \rho_{0} \dot{\chi}_{\ell} \chi_{\ell} d x_{2}=-\frac{1}{2} \int_{I_{2}} D_{1} \rho_{0} \cdot \chi_{\ell}^{2} d x_{2}
$$

more generally

$$
\int_{I_{2}} \rho_{0} \dot{\chi}_{\ell} \chi_{k} d x_{2}+\int_{I_{2}} \rho_{0} \dot{\chi}_{k} \chi_{\ell} d x_{2}=-\int_{I_{2}} D_{1} \rho_{0} \cdot \chi_{\ell} \chi_{k} d x_{2} \text { for any } k \geq 1 .
$$

Proof. Formula (4.26) is another form of (4.19). Formula (4.25) is its particular case for $k=\ell$.

By differentiating in $x_{1}$ the second orthogonality relation (4.6) for $k=\ell$, we obtain

$$
\dot{\alpha}_{\ell}=2 \mathcal{L}_{0 I_{2}}\left(\chi_{\ell}, \dot{\chi}_{\ell}\right)+\dot{\mathcal{L}}_{0 I_{2}}\left(\chi_{\ell}, \chi_{\ell}\right) .
$$

Applying the integral identity (4.3) for $\chi=\chi_{\ell}$ and $\zeta=\dot{\chi}_{\ell}$ and formula (4.25), we obtain

$$
\mathcal{L}_{0 I_{2}}\left(\chi_{\ell}, \dot{\chi}_{\ell}\right)=\alpha_{\ell} \int_{I_{2}} \rho_{0} \chi_{\ell} \dot{\chi}_{\ell} d x_{2}=-\frac{\alpha_{\ell}}{2} \int_{I_{2}} D_{1} \rho_{0} \cdot \chi_{\ell}^{2} d x_{2},
$$

which leads to (4.21).

The integral identity (4.24) is derived by differentiating (4.3) with respect to $x_{1}$ for $\chi=\chi_{\ell}$ and $\alpha=\alpha_{\ell}$.

The property $F_{\ell} \in H^{-1}\left(I_{2}\right)$ follows from the bounds, for any $\chi, \zeta \in H_{0}^{1}\left(I_{2}\right)$,

$$
\left|\dot{\mathcal{L}}_{0 I_{2}}(\chi, \zeta)\right| \leq K_{12}\left\|\sqrt{\kappa_{0}} D_{2} \chi\right\|_{I_{2}}\left\|\sqrt{\kappa_{0}} D_{2} \zeta\right\|_{I_{2}},
$$


compare with the second inequality (3.34), and similarly

$$
\left|\int_{I_{2}} D_{1} \rho_{0} \cdot \chi \zeta d x_{2}\right| \leq K_{13}\left\|\sqrt{\rho_{0}} \chi\right\|_{I_{2}}\left\|\sqrt{\rho_{0}} \zeta\right\|_{I_{2}} .
$$

Proposition 4.7. The degenerate BVP (4.22), (4.23) under the additional condition (4.25) has a unique weak solution. The coefficients of its Fourier expansion

$$
\dot{\chi}_{\ell}=\sum_{k=1}^{\infty} \dot{\chi}_{\ell, k} \chi_{k}
$$

can be calculated as

$$
\dot{\chi}_{\ell, k}=\frac{F_{\ell, k}}{\alpha_{k}-\alpha_{\ell}} \text { for } k \neq \ell
$$

with

$$
F_{\ell, k}:=\left\langle F_{\ell}, \chi_{k}\right\rangle_{I_{2}}= \begin{cases}-\dot{\mathcal{L}}_{0 I_{2}}\left(\chi_{k}, \chi_{\ell}\right)+\alpha_{\ell} \int_{I_{2}} D_{1} \rho_{0} \cdot \chi_{k} \chi_{\ell} d x_{2} & \text { for } k \neq \ell, \\ 0 & \text { for } k=\ell\end{cases}
$$

for $k=\ell$ see (4.25). Moreover, the following bounds hold:

$$
\left|F_{\ell, k}\right| \leq K_{12} \sqrt{\alpha_{k} \alpha_{\ell}}+K_{13} \alpha_{\ell}, \quad\left|\dot{\chi}_{\ell, \ell}\right| \leq \frac{1}{2} K_{13} \text { for any } k, \ell
$$

Proof. Formula (4.31) follows from (4.4) for $k \neq \ell$ and from (4.21) for $k=\ell$.

The equality $\left\langle F_{\ell}, \chi_{\ell}\right\rangle_{I_{2}}=0$ is the necessary condition for solvability of the degenerate BVP (4.22), (4.23). Moreover, its weak solution exists and is unique provided that $\int_{I_{2}} \rho_{0} \dot{\chi}_{\ell} \chi_{\ell} d x_{2}$ is given (see a general theory in [13]).

Setting $\zeta=\chi_{k}$ into (4.24) and applying (4.3) for $\chi=\chi_{k}, \alpha=\alpha_{k}$ and $\zeta=\dot{\chi}_{\ell}$, we obtain (4.30).

Bound (4.32) follows from (4.27), (4.28) together with equalities (4.5) and (4.6) for $k=\ell$.

Now we assume the following spectral gap property

$$
\sqrt{\alpha_{\ell}\left(x_{1}\right)}-\sqrt{\alpha_{k}\left(x_{1}\right)} \geq \delta_{s}(\ell-k) \text { for any } 1 \leq k<\ell \text { and a.a. } x_{1} \in I_{1},
$$

for some $\delta_{s}>0$ (we omit its lengthy proof here). Let $K_{14}:=\max \left\{K_{12}, K_{13}\right\}$.

Proposition 4.8. The following bound holds

$$
\left\|\sqrt{\rho_{0}} \dot{\chi}_{\ell}\left(x_{1}, \cdot\right)\right\|_{L^{2}\left(I_{1}\right)} \leq K_{15}\left(K_{10}, K_{14}, \delta_{s}\right) \sqrt{\alpha_{\ell}\left(x_{1}\right)} \text { for a.a. } x_{1} \in I_{1} .
$$

Consequently, $K_{8, N} \leq K_{6}\left(K_{15}^{2} N+\bar{\rho}_{0}\right)$ (in this respect, see Proposition 3.17).

Proof. Applying Proposition 4.7, we obtain

$$
\left\|\sqrt{\rho_{0}} \dot{\chi}_{\ell}\right\|_{I_{2}}^{2}=\sum_{k \geq 1 ; k \neq \ell}\left(\frac{F_{\ell, k}}{\alpha_{k}-\alpha_{\ell}}\right)^{2}+\left|\dot{\chi}_{\ell, \ell}\right|^{2} \leq K_{14}^{2}\left(\alpha_{\ell} \sum_{k \geq 1 ; k \neq \ell} \frac{1}{\left(\sqrt{\alpha_{k}}-\sqrt{\alpha_{\ell}}\right)^{2}}+\frac{1}{4}\right) .
$$


The spectral gap property (4.33) implies that

$$
\sum_{k \geq 1 ; k \neq \ell} \frac{1}{\left(\sqrt{\alpha_{k}}-\sqrt{\alpha_{\ell}}\right)^{2}} \leq \frac{1}{\delta_{s}^{2}}\left(\sum_{k=1}^{\ell-1} \frac{1}{(\ell-k)^{2}}+\sum_{k=\ell+1}^{\infty} \frac{1}{(k-\ell)^{2}}\right)<\frac{2}{\delta_{s}^{2}} \sum_{m=1}^{\infty} \frac{1}{m^{2}}=\frac{\pi^{2}}{3 \delta_{s}^{2}}
$$

Invoking the lower bound for $\alpha_{\ell}$ in (4.11), we complete the proof of (4.34).

Concerning the bound for $K_{8, N}$, from (4.12) we have

$$
\int_{I_{2}}\left(G^{(1)^{-1}} D_{1} \chi, D_{1} \chi\right)_{\mathbb{C}^{N}} \rho_{0} d x_{2}=\sum_{\ell=1}^{N} \alpha_{\ell}^{-1}\left\|\sqrt{\rho_{0}} \dot{\chi}_{\ell}\right\|_{I_{2}}^{2} \leq K_{15}^{2} N
$$

REMARK 4.9. In the particular case of $\kappa_{0}$ and $\rho_{0}$ independent of $x_{1}$, the results of Proposition 4.8 can be essentially improved. Namely, we first get (compare with $(4.32))$

$$
\left|F_{\ell, k}\right| \leq\left\|D_{1} V_{0}\right\|_{\tilde{L}^{1, \infty}(\Omega)}\left\|\chi_{\ell}\right\|_{L^{\infty}\left(I_{2}\right)}\left\|\chi_{k}\right\|_{L^{\infty}\left(I_{2}\right)} \leq \tilde{K}_{12} \sqrt[4]{\alpha_{k} \alpha_{\ell}}, \text { for any } k, \ell
$$

with $\tilde{K}_{12}:=2 \mu_{1,0} / \sqrt{\underline{\rho}_{0} \underline{\kappa}_{0}}$, since

$$
\left\|\chi_{\ell}\right\|_{L^{\infty}\left(I_{2}\right)}^{2} \leq 2 \int_{I_{2}}\left|\chi_{\ell}\right|\left|D_{2} \chi_{\ell}\right| d x_{2} \leq \frac{2}{\sqrt{\underline{\rho}_{0} \underline{\kappa}_{0}}}\left\|\sqrt{\rho_{0}} \chi_{\ell}\right\|_{I_{2}}\left\|\sqrt{\kappa_{0}} D_{2} \chi_{\ell}\right\|_{I_{2}} \leq \frac{2}{\sqrt{\underline{\rho}_{0} \underline{\kappa}_{0}}} \sqrt{\alpha_{\ell}} .
$$

Using the obvious inequality $\sqrt[4]{\alpha_{k} \alpha_{\ell}} \leq\left(\sqrt{\alpha_{k}}+\sqrt{\alpha_{\ell}}\right) / 2$, now we derive the uniform in $\ell \geq 1$ bound

$$
\left\|\sqrt{\rho_{0}} \dot{\chi}_{\ell}\right\|_{I_{2}} \leq \frac{\tilde{K}_{12}}{2}\left(\frac{\pi}{\sqrt{3} \delta_{s}}+1\right)=: \tilde{K}_{15}
$$

and then (applying the lower bound in (4.11)) the uniform in $N \geq 1$ bound

$$
K_{8, N} \leq K_{6}\left(\frac{\pi^{2}}{6} K_{10} \tilde{K}_{15}^{2}+\bar{\rho}_{0}\right)
$$

Thus the $H_{0}^{1}\left(I_{1}\right)$-positive definiteness property (3.35) becomes the best possible.

The results of the rest of the section could be improved in this particular case as well.

LEMMA 4.10. The following bound holds

$$
\left\|s_{2 N}^{(\theta)}\right\|_{L^{\infty}\left(I_{1}\right)} \leq \frac{K_{14}}{\delta_{s}} \underline{\alpha}_{N+1}^{-(\theta-1) / 2}\left(c_{0}+\log ^{1 / 2} N\right) \quad \text { for } \theta \geq 1
$$

where $c_{0}$ is a generic numerical constant.

Proof. Applying once more Proposition 4.7 and the spectral gap property, similarly to the previous proof we obtain

$$
\begin{aligned}
{\left[s_{2 N}^{(\theta)}\right]^{2}=\sum_{\ell=N+1}^{\infty} \alpha_{\ell}^{-\theta} \sum_{k=1}^{N}\left(\frac{F_{\ell, k}}{\alpha_{k}-\alpha_{\ell}}\right)^{2} } & \leq\left(\frac{K_{14}}{\delta_{s}}\right)^{2} \sum_{\ell=N+1}^{\infty} \alpha_{\ell}^{-(\theta-1)} \sum_{k=1}^{N} \frac{1}{(\ell-k)^{2}} \\
& =\left(\frac{K_{14}}{\delta_{s}}\right)^{2} \sum_{n=1}^{\infty} \alpha_{n+N}^{-(\theta-1)} \sum_{m=n}^{n+N-1} \frac{1}{m^{2}}
\end{aligned}
$$


For $\theta \geq 1$, we obtain

$$
\left[s_{2 N}^{(\theta)}\right]^{2} \leq\left(\frac{K_{14}}{\delta_{s}}\right)^{2} \alpha_{N+1}^{-(\theta-1)} S_{1 N}, \text { with } S_{1 N}:=\sum_{n=1}^{\infty} \sum_{m=n}^{n+N-1} \frac{1}{m^{2}} .
$$

Since

$$
\frac{1}{m^{2}}<\frac{1}{m-1}-\frac{1}{m} \text { for } m \geq 2
$$

we have

$$
\sum_{m=n}^{n+N-1} \frac{1}{m^{2}}<\frac{1}{n-1}-\frac{1}{n+N-1} \text { for } n \geq 2
$$

and thus

$$
S_{1 N}<\frac{\pi^{2}}{6}+\sum_{k=1}^{N} \frac{1}{k} \leq c_{0}+\log N
$$

which completes the proof.

We now estimate $s_{1 N}^{(\theta)}$ under the low regularity (with respect to $x_{1}$ ) of coefficients $\kappa_{0}, V_{0}$ and $\rho_{0}$, see $(4.20)$.

Lemma 4.11. The following bounds hold

$$
\begin{aligned}
& \left\|s_{1 N}^{(\theta)}\right\|_{L^{\infty}\left(I_{1}\right)} \leq 2 K_{10}^{(\theta-1) / 2} \frac{K_{14}}{\delta_{s}}(N+1)^{-(\theta-1)}\left(1+\log ^{1 / 2} N\right) \quad \text { for } 1 \leq \theta \leq 3 / 2, \\
& \left\|s_{1 N}^{(\theta)}\right\|_{L^{\infty}\left(I_{1}\right)} \leq c_{\theta} K_{10}^{(\theta-1) / 2} \frac{K_{14}}{\delta_{s}}(N+1)^{-1 / 2} \text { for } \theta>3 / 2,
\end{aligned}
$$

where $c_{\theta}$ depends only on $\theta$.

Proof. Similar to relations (4.35), we obtain

$$
\left[s_{1 N}^{(\theta)}\right]^{2}=\sum_{\ell=1}^{N} \alpha_{\ell}^{-\theta} \sum_{k=N+1}^{\infty}\left(\frac{F_{\ell, k}}{\alpha_{k}-\alpha_{\ell}}\right)^{2} \leq\left(\frac{K_{14}}{\delta_{s}}\right)^{2} \sum_{\ell=1}^{N} \alpha_{\ell}^{-(\theta-1)} \sum_{m=1}^{\infty} \frac{1}{(m+N-\ell)^{2}}
$$

Inequality (4.36) implies that

$$
\sum_{m=1}^{\infty} \frac{1}{(m+N-\ell)^{2}}<\frac{1}{(1+N-\ell)^{2}}+\frac{1}{1+N-\ell} \leq \frac{2}{N+1-\ell}
$$

Therefore, for $\theta \geq 1$, exploiting the lower bound in (4.11), we obtain

$$
\left[s_{1 N}^{(\theta)}\right]^{2}<2\left(\frac{K_{14}}{\delta_{s}}\right)^{2} K_{10}^{\theta-1} S_{2 N}(\tilde{\theta})
$$

with $S_{2 N}(\tilde{\theta}):=\sum_{\ell=1}^{N} \frac{1}{\ell^{2 \tilde{\theta}}(N+1-\ell)}$ and $\tilde{\theta}:=\theta-1$. 
In the case where $\tilde{\theta} \in[0,1 / 2]$, exploiting the Hölder inequality for sums we have

$$
S_{2 N}(\tilde{\theta}) \leq S_{2 N}^{1-2 \tilde{\theta}}(0) S_{2 N}^{2 \tilde{\theta}}\left(\frac{1}{2}\right) .
$$

Since $S_{2 N}(0)<1+\log N$ and

$$
S_{2 N}\left(\frac{1}{2}\right) \leq \sum_{\ell=1}^{N} \frac{1}{\ell(N+1-\ell)}=\frac{1}{N+1} \sum_{\ell=1}^{N} \frac{1}{\ell}+\frac{1}{N+1-\ell}<\frac{2}{N+1}(1+\log N),
$$

we derive

$$
S_{2 N}(\tilde{\theta})<2^{2 \tilde{\theta}}(N+1)^{-2 \tilde{\theta}}(1+\log N) .
$$

In the case $\tilde{\theta}=(1+\varepsilon) / 2$ with $\varepsilon>0$, we obtain

$$
S_{2 N}(\tilde{\theta})=h^{1+\varepsilon} \sum_{\ell=1}^{N} g_{\varepsilon}(\ell h) h<h^{1+\varepsilon} \int_{h / 2}^{1-h / 2} g_{\varepsilon}(\xi) d \xi
$$

with $h:=\frac{1}{N+1}$ and $g_{\varepsilon}(\xi):=\frac{1}{\xi^{1+\varepsilon}(1-\xi)}$. Here we have taken into account that the sum is the compound midpoint quadrature formula for the integral and $g_{\varepsilon}^{\prime \prime}>0$ on $(0,1)$. Furthermore, we can estimate the integral as follows

$$
\begin{array}{r}
h^{1+\varepsilon} \int_{h / 2}^{1-h / 2} g_{\varepsilon}(\xi) d \xi<h^{1+\varepsilon}\left(2 \int_{h / 2}^{1 / 2} \frac{d \xi}{\xi^{1+\varepsilon}}+2^{1+\varepsilon} \int_{h / 2}^{1 / 2} \frac{d \xi}{1-\xi}\right) \\
<h^{1+\varepsilon}\left[\frac{2}{\varepsilon}\left(\frac{h}{2}\right)^{-\varepsilon}+2^{1+\varepsilon} \log \frac{1}{h}\right]=2^{1+\varepsilon}\left(\frac{1}{\varepsilon}+h^{\varepsilon} \log \frac{1}{h}\right) h .
\end{array}
$$

Consequently

$$
S_{2 N}(\tilde{\theta}) \leq c_{\varepsilon}(N+1)^{-1} .
$$

Inserting the bounds for $S_{2 N}(\tilde{\theta})$ into (4.38), we complete the proof.

In order to obtain higher-order bounds than in Lemma 4.11, we need an additional condition on $F_{\ell}$ (finally, on $\kappa_{0}, V_{0}$ and $\rho_{0}$ ). To state this, we go back to the definition of the spaces $\mathbf{H}^{0, \theta}(\Omega)$ and introduce a similar family of Hilbert spaces of functions depending only on $x_{2}$, for a.a. $x_{1} \in I_{1}$

$$
\mathbf{H}^{\theta}\left[x_{1}\right]\left(I_{2}\right):=\left\{\zeta \in L^{2}\left(I_{2}\right) ;\|\zeta\|_{\mathbf{H}^{\theta}\left[x_{1}\right]\left(I_{2}\right)}:=\left[\sum_{\ell=1}^{\infty} \alpha_{\ell}^{\theta}\left(x_{1}\right)\left|\tilde{\zeta}_{\ell}\left(x_{1}\right)\right|^{2}\right]^{1 / 2}<\infty\right\}, \quad \theta \geq 0 ;
$$

note that the Fourier coefficients $\tilde{\zeta}_{\ell}$, see (4.7), are $x_{1}$-dependent even for $\zeta$ independent of $x_{1}$.

Let $0 \leq \theta_{0}<\theta_{1}$. Clearly $\mathbf{H}^{\theta_{1}}\left(I_{2}\right) \subset \mathbf{H}^{\theta_{0}}\left(I_{2}\right)$ and $\mathbf{H}^{\theta_{1}}\left(I_{2}\right)$ is dense in $\mathbf{H}^{\theta_{0}}\left(I_{2}\right)$; hereafter we omit the symbol $\left[x_{1}\right]$ for brevity. Moreover, the Hölder inequality for series implies the following multiplicative inequality, for $\theta_{0} \leq \theta \leq \theta_{1}$

$$
\|\zeta\|_{\mathbf{H}^{\theta}\left(I_{2}\right)} \leq\|\zeta\|_{\mathbf{H}^{\theta_{0}\left(I_{2}\right)}}^{\frac{\theta_{1}-\theta}{\theta_{1}-\theta_{0}}}\|\zeta\|_{\mathbf{H}^{\theta_{1}\left(I_{2}\right)}}^{\frac{\theta-\theta_{0}}{\theta_{1}-\theta_{0}}} \quad \text { for any } \zeta \in \mathbf{H}^{\theta_{1}}\left(I_{2}\right)
$$


We have $\mathbf{H}^{0}\left(I_{2}\right)=L^{2}\left(I_{2}\right)$ and $\mathbf{H}^{1}\left(I_{2}\right)=H_{0}^{1}\left(I_{2}\right)$, up to an equivalence of norms (uniformly in $x_{1} \in I_{1}$ ), and

$$
\|\zeta\|_{\mathbf{H}^{0}\left(I_{2}\right)}=\left\|\sqrt{\rho_{0}} \zeta\right\|_{L^{2}\left(I_{2}\right)}, \quad\|\zeta\|_{\mathbf{H}^{1}\left(I_{2}\right)}=\left[\mathcal{L}_{0 I_{2}}(\zeta, \zeta)\right]^{1 / 2} .
$$

For completeness of our error bounds, we also need to consider functions $\zeta \in$ $H^{-1}\left(I_{2}\right)$ such that

$$
\left\|\rho_{0}^{-1} \zeta\right\|_{\mathbf{H}^{-\theta}\left(I_{2}\right)}:=\sup _{\|\eta\|_{\mathbf{H}^{\theta}\left(I_{2}\right)}=1}\left|\langle\zeta, \eta\rangle_{I_{2}}\right|<\infty \text { for } 0<\theta \leq 1 .
$$

For $\theta=1$, this norm is equivalent (uniformly in $x_{1} \in I_{1}$ ) to the norm $\|\zeta\|_{H^{-1}\left(I_{2}\right)}$. Note that in general here $\rho_{0}^{-1} \zeta$ is only a convenient notation rather than the product of $\rho_{0}^{-1}$ and $\zeta$ (though under condition (4.20) on $\rho_{0}$ this is well-defined). On the other hand, for any $\zeta \in L^{2}\left(I_{2}\right)$

$$
\left\|\rho_{0}^{-1}\left(\rho_{0} \zeta\right)\right\|_{\mathbf{H}^{-\theta}\left(I_{2}\right)}=\|\zeta\|_{\mathbf{H}^{-\theta}\left(I_{2}\right)}=\left[\sum_{\ell=1}^{\infty} \alpha_{\ell}^{-\theta}\left|\tilde{\zeta}_{\ell}\right|^{2}\right]^{1 / 2} .
$$

We introduce the following condition on $F_{\ell}, \ell \geq 1$ :

$$
\operatorname{ess} \sup _{x_{1} \in I_{1}} \alpha_{\ell}^{-\beta / 2-1}\left(x_{1}\right)\left\|\left(\rho_{0}^{-1}\left(F_{\ell}-\dot{\alpha}_{\ell} \rho_{0} \chi_{\ell}\right)\right)\left(x_{1}, \cdot\right)\right\|_{\mathbf{H}^{\beta}\left[x_{1}\right]\left(I_{2}\right)} \leq 2 K_{16, \beta}
$$

for some $\beta \geq-1$. This follows from

$$
\left\|\rho_{0}^{-1} \dot{\mathcal{H}}_{0} \chi_{\ell}\right\|_{\mathbf{H}^{\beta}\left(I_{2}\right)} \leq K_{16, \beta} \alpha_{\ell}^{\beta / 2+1},\left\|\rho_{0}^{(1)} \chi_{\ell}\right\|_{\mathbf{H}^{\beta}\left(I_{2}\right)} \leq K_{16, \beta} \alpha_{\ell}^{\beta / 2} \text { on } I_{1} \text {, for } \ell \geq 1
$$

Notice that $\left\|\rho_{0}^{-1} \mathcal{H}_{0} \chi_{\ell}\right\|_{\mathbf{H}^{\beta}\left(I_{2}\right)}=\alpha_{\ell}\left\|\chi_{\ell}\right\|_{\mathbf{H}^{\beta}\left(I_{2}\right)}=\alpha_{\ell}^{\beta / 2+1}$, for $\beta \geq-1$ and $\ell \geq 1$.

The multiplicative inequality (4.40) implies that if one of the conditions is valid for some $\beta=\beta_{0}, \beta_{1}$ such that $-1 \leq \beta_{0}<\beta_{1}$, then this is also valid for any $\beta_{0} \leq \beta \leq \beta_{1}$ (with $K_{16, \beta}=K_{16, \beta_{0}}^{\frac{\beta_{1}-\beta}{\beta_{1}-\beta_{0}}} K_{16, \beta_{1}}^{\frac{\beta-\beta_{0}}{\beta_{1}-\beta_{0}}}$ ). In particular, it is sufficient to consider only integer values of $\beta$.

For $\beta=-1$ and $D_{2} \rho_{0}^{(1)} \in \tilde{L}^{2, \infty}(\Omega)$, using bound (4.27) and taking into account that $\mathbf{H}^{1}\left(I_{2}\right)=H_{0}^{1}\left(I_{2}\right)$ up to an equivalence of norms, we have

$$
\begin{aligned}
\left|\left\langle\dot{\mathcal{H}}_{0} \chi_{\ell}, \eta\right\rangle_{I_{2}}\right| & =\left|\dot{\mathcal{L}}_{0 I_{2}}\left(\chi_{\ell}, \eta\right)\right| \leq K_{12}\left\|\sqrt{\kappa_{0}} D_{2} \chi_{\ell}\right\|_{I_{2}}\left\|\sqrt{\kappa_{0}} D_{2} \eta\right\|_{I_{2}} \leq K_{12} \sqrt{\alpha_{\ell}}\|\eta\|_{\mathbf{H}^{1}\left(I_{2}\right)}, \\
\left|\int_{I_{2}} \rho_{0} \rho_{0}^{(1)} \chi_{\ell} \eta d x_{2}\right| & \leq\left\|\chi_{\ell}\right\|_{\mathbf{H}^{-1}\left(I_{2}\right)}\left\|\rho_{0}^{(1)} \eta\right\|_{\mathbf{H}^{1}\left(I_{2}\right)} \\
& \leq \frac{1}{\sqrt{\alpha_{\ell}}} \sqrt{\frac{2 K_{6} X_{2}}{\underline{\kappa}_{0}}}\left\|D_{2} \rho_{0}^{(1)}\right\|_{\tilde{L}^{2, \infty}(\Omega)}\|\eta\|_{\mathbf{H}^{1}\left(I_{2}\right)},
\end{aligned}
$$

for any $\eta \in H_{0}^{1}\left(I_{2}\right)$. Thus condition (4.42) holds for $\beta=-1$.

For $\beta=0$ and $D_{2} \kappa_{0}^{(1)}, V_{0}, D_{1} V_{0} \in \tilde{L}^{2, \infty}(\Omega)$, we obtain

$$
\dot{\mathcal{H}}_{0} \chi_{\ell}=-\kappa_{0}^{(1)} D_{2}\left(\kappa_{0} D_{2} \chi_{\ell}\right)-D_{2} \kappa_{0}^{(1)} \cdot \kappa_{0} D_{2} \chi_{\ell}+D_{1} V_{0} \cdot \chi_{\ell}
$$


Consequently

$$
\begin{aligned}
\left\|\rho_{0}^{-1} \dot{\mathcal{H}}_{0} \chi_{\ell}\right\|_{\mathbf{H}^{0}\left(I_{2}\right)}= & \left\|\frac{1}{\sqrt{\rho_{0}}} \dot{\mathcal{H}}_{0} \chi_{\ell}\right\|_{I_{2}} \\
\leq & \frac{1}{\sqrt{\overline{\rho_{0}}}}\left[\left(\left\|\kappa_{0}^{(1)}\right\|_{L^{\infty}(\Omega)}+\sqrt{X_{2}}\left\|D_{2} \kappa_{0}^{(1)}\right\|_{\tilde{L}^{2, \infty}(\Omega)}\right)\left\|D_{2}\left(\kappa_{0} D_{2} \chi_{\ell}\right)\right\|_{I_{2}}\right. \\
& \left.+\left\|D_{1} V_{0}\right\|_{\tilde{L}^{2, \infty}(\Omega)}\left\|\chi_{\ell}\right\|_{L^{\infty}(\Omega)}\right] .
\end{aligned}
$$

Since $D_{2}\left(\kappa_{0} D_{2} \chi_{\ell}\right)=\left(V_{0}-\alpha_{\ell} \rho_{\ell}\right) \chi_{\ell}$ also and

$$
\left\|\rho_{0}^{(1)} \chi_{\ell}\right\|_{\mathbf{H}^{0}\left(I_{2}\right)} \leq\left\|\rho_{0}^{(1)}\right\|_{L^{\infty}(\Omega)}\left\|\sqrt{\rho_{0}} \chi_{\ell}\right\|_{I_{2}}=\left\|\rho_{0}^{(1)}\right\|_{L^{\infty}(\Omega)},
$$

condition (4.42) holds for $\beta=0$ as well.

LEMma 4.12. Let $\theta>3 / 2$ and condition (4.42) for $\beta=\theta-5 / 2$ be valid. Then

$$
\left\|s_{1 N}^{(\theta)}\right\|_{L^{\infty}\left(I_{1}\right)} \leq 2 \sqrt{\frac{2}{\delta_{s}}} K_{10}^{1 / 4} K_{16, \beta} \underline{\alpha}_{N+1}^{-(\theta-3 / 2) / 2}(N+1)^{-1 / 2}\left(1+\log ^{1 / 2} N\right) .
$$

Proof. Using the spectral gap property (4.33), we can replace the inequality in (4.37) by

$$
\left[s_{1 N}^{(\theta)}\right]^{2} \leq \sum_{\ell=1}^{N} \alpha_{\ell}^{-\theta} \frac{1}{\delta_{s}(N+1-\ell)} \sum_{k=N+1}^{\infty} \alpha_{k}^{-1} F_{\ell, k}^{2} .
$$

Estimating the right hand side and applying condition (4.42) for some $\beta \geq-1$, we obtain

$$
\begin{aligned}
{\left[s_{1 N}^{(\theta)}\right]^{2} } & \leq \frac{1}{\delta_{s}} \alpha_{N+1}^{-(\beta+1)} \sum_{\ell=1}^{N} \alpha_{\ell}^{-\theta} \frac{1}{N+1-\ell} \sum_{k=N+1}^{\infty} \alpha_{k}^{\beta} F_{\ell, k}^{2} \\
& \leq \frac{1}{\delta_{s} \alpha_{N+1}^{(\beta+1)}} \sum_{\ell=1}^{N} \alpha_{\ell}^{-\theta}\left\|\rho_{0}^{-1}\left(F_{\ell}-\dot{\alpha}_{\ell} \rho_{0} \chi \ell\right)\right\|_{\mathbf{H}^{\beta}\left(I_{2}\right)}^{2} \frac{1}{N+1-\ell} \\
& \leq \frac{\left(2 K_{16, \beta}\right)^{2}}{\delta_{s} \alpha_{N+1}^{(\beta+1)}} \sum_{\ell=1}^{N} \alpha_{\ell}^{\beta+2-\theta} \frac{1}{N+1-\ell} .
\end{aligned}
$$

Setting $\beta:=\theta-5 / 2$ and applying the lower bound in (4.11) and bound (4.39), we complete the proof.

REMARK 4.13. Since $k>\ell$ in (4.43), we have actually exploited only the weakened version of condition (4.41) with $\rho_{0}^{-1} F_{\ell}-\mathcal{P}_{\ell}\left(\rho_{0}^{-1} F_{\ell}\right)$ replacing $\rho_{0}^{-1}\left(F_{\ell}-\dot{\alpha}_{\ell} \rho_{0} \chi_{\ell}\right)$.

Summarizing the results of Proposition 4.2 (see (4.15)), Corollary 4.4 and Lemmas 4.10-4.12, we obtain the following proposition.

Proposition 4.14. Let conditions (4.20) and (4.33) be valid. Also let $w \in \mathbf{H}^{0, \theta}(\Omega)$ and $D_{1} w \in \mathbf{H}^{0, \theta-1}(\Omega)$ for some $\theta>1$ and, in the case where $\theta>3 / 2$, let condition (4.42) for $\beta=\theta-5 / 2$ be valid too. Then the following $H^{1}(\Omega)$-approximation bound holds:

$$
\left\|w-\mathcal{P}_{N} w\right\|_{H^{1}(\Omega)} \leq K_{17}(N+1)^{-(\theta-1)}\left(1+\log ^{1 / 2} N\right)\left(\|w\|_{\mathbf{H}^{0, \theta}(\Omega)}+\left\|D_{1} w\right\|_{\mathbf{H}^{0, \theta-1}(\Omega)}\right) .
$$


Corollary 4.15. Let conditions (4.20) and (4.33) be valid. Also let $u \in \mathbf{H}^{0, \theta}(\Omega)$ and $D_{1} u \in \mathbf{H}^{0, \theta-1}(\Omega)$ for some $\theta>1$ and, in the case where $\theta>3 / 2$, let condition (4.42) for $\beta=\theta-5 / 2$ be valid as well. Then the following $H^{1}(\Omega)$-error bound holds:

$$
\left\|u-v^{(N)}\right\|_{H^{1}(\Omega)} \leq K_{18}(N+1)^{-(\theta-1)}\left(1+\log ^{1 / 2} N\right)\left(\|u\|_{\mathbf{H}^{0, \theta}(\Omega)}+\left\|D_{1} u\right\|_{\mathbf{H}^{0, \theta-1}(\Omega)}\right) .
$$

Under additional regularity conditions (2.8) and

$$
D_{2} \kappa_{0}^{(1)}, V_{0}, D_{1} V_{0}, D_{2} \rho_{0}^{(1)} \in \tilde{L}^{2, \infty}(\Omega),
$$

the following $L^{2}(\Omega)$-error bound holds as well:

$$
\left\|u-v^{(N)}\right\|_{L^{2}(\Omega)} \leq K_{19}(N+1)^{-\theta}(1+\log N)\left(\|u\|_{\mathbf{H}^{0, \theta}(\Omega)}+\left\|D_{1} u\right\|_{\mathbf{H}^{0, \theta-1}(\Omega)}\right) .
$$

Proof. The results are straightforward consequences of Propositions 3.20 and 4.14. Concerning bound (4.47), we apply bound (4.44) together with its version for $\theta=2$, also exploiting bound (2.9) and condition (4.42) for $\beta=-1 / 2$ (as a consequence of the same condition verified above for $\beta=-1,0)$.

REMARK 4.16. If for $\theta>3 / 2$ condition (4.42) is not imposed, then bounds (4.44) and (4.45) with $c_{\theta}(N+1)^{-1 / 2}$ replacing $(N+1)^{-(\theta-1)}\left(1+\log ^{1 / 2} N\right)$ additionally hold, due to the second bound in Lemma 4.11.

\section{A discretized Galerkin method}

To get a practically implementable method, it is necessary to discretize in $x_{1}$ the Galerkin BVP (3.24), (3.25). This can be accomplished in many ways. In order to preserve Hermitian symmetry and positive definiteness properties, one natural approach consists in applying the finite element method.

We consider only its simplest version based on linear elements with numerical integration in $x_{1}$ which is closer to practice. Let $x_{10}=0<x_{11}<\cdots<x_{1 \bar{m}}=X_{1}$ be a general mesh $\bar{\omega}_{h}$ on $\bar{I}_{1}$, with steps $h_{m}:=x_{1 m}-x_{1 m-1}$. Let $S_{h}\left(\bar{I}_{1}\right)$ be the space of continuous functions on $\bar{I}_{1}$, linear on each element $\left[x_{1 m-1}, x_{1 m}\right]$ and equal to zero at $x_{1}=0, X_{1}$. Clearly $\operatorname{dim} S_{h}\left(\bar{I}_{1}\right)=\bar{m}-1$ and $S_{h}\left(\bar{I}_{1}\right) \subset H_{0}^{1}\left(I_{1}\right)$.

Let $\omega_{h}^{*}=\left\{x_{1 m-1 / 2}\right\}_{m=1}^{\bar{m}}$ be another mesh with the nodes $x_{1 m-1 / 2}:=\left(x_{1 m-1}+\right.$ $\left.x_{1 m}\right) / 2$ and steps $h_{m+1 / 2}:=x_{1 m+1 / 2}-x_{1 m-1 / 2}=\left(h_{m}+h_{m+1}\right) / 2$.

We seek an approximate solution of the BVP (2.1), (2.2) in the form

$$
v_{h}^{(N)}\left(x_{1}, x_{2}\right)=\sum_{\ell=1}^{N} c_{h \ell}\left(x_{1}\right) \chi_{\ell}\left(x_{1}, x_{2}\right)=\sum_{m=1}^{\bar{m}-1} \sum_{\ell=1}^{N} c_{h \ell}\left(x_{1 m}\right) e_{h m}\left(x_{1}\right) \chi_{\ell}\left(x_{1}, x_{2}\right),
$$

where $c_{h \ell} \in S_{h}\left(\bar{I}_{1}\right)$ and the "hat" functions

$$
e_{h m}\left(x_{1}\right)= \begin{cases}\left(x_{1}-x_{1 m-1}\right) / h_{m} & \text { for } x_{1 m-1} \leq x_{1} \leq x_{1 m}, \\ \left(x_{1 m+1}-x_{1}\right) / h_{m+1} & \text { for } x_{1 m} \leq x_{1} \leq x_{1 m+1}, \\ 0 & \text { for } x_{1} \notin\left[x_{1 m-1}, x_{1 m+1}\right],\end{cases}
$$

for $1 \leq m \leq \bar{m}-1$, form the standard basis in $S_{h}\left(\bar{I}_{1}\right)$. We denote by $S_{h}^{N}$ the space of all functions on $\bar{\Omega}$ having form (5.1). Clearly $\operatorname{dim} S_{h}^{N}=(\bar{m}-1) N$ and $S_{h}^{N} \subset H_{0}^{1}(\Omega)$. 
We approximate the original integral identity (2.4) as follows:

$$
\mathcal{L}_{\Omega h}\left(v_{h}^{(N)}, \varphi\right)=\ell_{\Omega h}(\varphi) \text { for any } \varphi \in S_{h}^{N}
$$

(compare with the semi-discrete version (2.17)), where

$$
\begin{aligned}
\mathcal{L}_{\Omega h}(w, \varphi) & :=\sum_{m=1}^{\bar{m}} \int_{I_{2}}\left(\sum_{i, j=1}^{2} \kappa_{i j} D_{j} w \cdot D_{i} \varphi^{*}+V w \varphi^{*}\right)\left(x_{1 m-1 / 2}, x_{2}\right) d x_{2} h_{m}, \\
\ell_{\Omega h}(\varphi) & :=\sum_{m=1}^{\bar{m}}\left\langle f\left(x_{1 m-1 / 2}, \cdot\right), \varphi\left(x_{1 m-1 / 2}, \cdot\right)\right\rangle_{I_{2}} h_{m} .
\end{aligned}
$$

In the above formulas, we have obviously approximated the integrals over $I_{1}$ by applying the compound midpoint quadrature formula. In this section we impose additional regularity conditions

$$
\begin{gathered}
D_{i} \chi_{\ell} \in C\left(\bar{I}_{1} ; L^{2}\left(I_{2}\right)\right), \kappa_{i j} \in C\left(\bar{I}_{1} ; L^{\infty}\left(I_{2}\right)\right) \\
\text { for all } i, j, V \in C\left(\bar{I}_{1} ; L^{1}\left(I_{2}\right)\right), f \in C\left(\bar{I}_{1} ; H^{-1}\left(I_{2}\right)\right)
\end{gathered}
$$

(compare with (3.32)). Note that only the values $\left.\chi_{\ell}\right|_{\omega_{h}^{*} \times I_{2}}$ (and $\left.D \chi_{\ell}\right|_{\omega_{h}^{*} \times I_{2}}$ ) of $\chi_{\ell}$ are involved in (5.2), for $1 \leq \ell \leq N$.

Clearly $\mathcal{L}_{\Omega h}(w, \varphi)$ is a bounded, Hermitian symmetric, and a positive definite sesquilinear form on $S_{h}^{N} \times S_{h}^{N}$. The last property means precisely that

$$
\nu\|w\|_{H_{0 h}^{1}(\Omega)}^{2} \leq \mathcal{L}_{\Omega h}(w, \varphi) \text { for any } w \in S_{h}^{N}
$$

where

$$
\|w\|_{H_{0 h}^{1}(\Omega)}:=\|\||| D w \mid\left\|_{L^{2}\left(I_{2}\right)}\right\|_{L_{h}^{2}\left(I_{1}\right)}, \quad\|y\|_{L_{h}^{2}\left(I_{1}\right)}:=\left(\sum_{m=1}^{\bar{m}}\left|y_{m-1 / 2}\right|^{2} h_{m}\right)^{1 / 2},
$$

with $y_{m-1 / 2}=y\left(x_{1 m-1 / 2}\right)$. Consequently, for any $f \in C\left(\bar{I}_{1} ; H^{-1}\left(I_{2}\right)\right)$, there exists a unique approximate solution $v_{h}^{(N)}$ which satisfies the bound

$$
\left\|v_{h}^{(N)}\right\|_{H_{0 h}^{1}(\Omega)} \leq \nu^{-1}\|\| f\left\|_{H^{-1}\left(I_{2}\right)}\right\|_{L_{h}^{2}\left(I_{1}\right)} ;
$$

compare with Corollary 3.4.

Now we need to introduce the difference and averaging mesh operators

$$
\begin{gathered}
\delta_{1} \eta_{m-1 / 2}:=\frac{\eta_{m}-\eta_{m-1}}{h_{m}}, \quad s_{1} \eta_{m-1 / 2}:=\frac{\eta_{m-1}+\eta_{m}}{2} \\
\delta_{1}^{*} y_{m}:=\frac{y_{m+1 / 2}-y_{m-1 / 2}}{h_{m+1 / 2}}, \quad s_{1}^{*} y_{m}:=\frac{h_{m} y_{m-1 / 2}+h_{m+1} y_{m+1 / 2}}{2 h_{m+1 / 2}},
\end{gathered}
$$

with $\eta_{m}=\eta\left(x_{1 m}\right)$. Also, let $H_{h}$ be the space of functions defined on the mesh $\bar{\omega}_{h}$ and having zero values at $x_{1}=0, X_{1}$ and $H_{h}^{N}:=\left[H_{h}\right]^{N}$.

Proposition 5.1. Let $v=v_{h}^{(N)}$ and $\varphi$ be any functions in $S_{h}^{N}$ having respective decompositions (5.1) and

$$
\varphi\left(x_{1}, x_{2}\right)=\sum_{\ell=1}^{N} d_{h \ell}\left(x_{1}\right) \chi_{\ell}\left(x_{1}, x_{2}\right)=\sum_{m=1}^{\bar{m}-1} \sum_{\ell=1}^{N} d_{h \ell}\left(x_{1 m}\right) e_{h m}\left(x_{1}\right) \chi_{\ell}\left(x_{1}, x_{2}\right),
$$


with $d_{h \ell} \in S_{h}\left(\bar{I}_{1}\right)$ for $1 \leq \ell \leq N$, and let $\mathbf{c}_{h}:=\left(c_{h 1}, \ldots, c_{h N}\right)^{T}$ and $\mathbf{d}_{h}:=\left(d_{h 1}, \ldots, d_{h N}\right)^{T}$ be corresponding vector-functions in $H_{h}^{N}$. Then the following identity holds:

$$
\begin{aligned}
\mathcal{L}_{\Omega h}(v, \varphi)= & \mathcal{L}_{I_{1} h}^{N}\left(\mathbf{c}_{h}, \mathbf{d}_{h}\right) \\
:= & \sum_{m=1}^{\bar{m}-1}\left[\left(A \delta_{1} \mathbf{c}_{h}+A^{(0)} s_{1} \mathbf{c}_{h}, \delta_{1} \mathbf{d}_{h}\right)_{\mathbb{C}^{N}}\right. \\
& \left.+\left(A^{(0)^{*}} \delta_{1} \mathbf{c}_{h}+B s_{1} \mathbf{c}_{h}, s_{1} \mathbf{d}_{h}\right)_{\mathbb{C}^{N}}\right]_{m-1 / 2} h_{m},
\end{aligned}
$$

where $A_{m-1 / 2}, A_{m-1 / 2}^{(0)}$ and $B_{m-1 / 2}$ are the values of the matrices introduced in Lemma 3.7.

Moreover, the following uniform bounds for the entries of the matrices hold, for any $1 \leq k \leq N$ and $1 \leq \ell \leq N$

$$
\left\|a_{k \ell}\right\|_{C\left(\bar{I}_{1}\right)}+\left\|a_{k \ell}^{(0)}\right\|_{C\left(\bar{I}_{1}\right)}+\left\|b_{k \ell}\right\|_{C\left(\bar{I}_{1}\right)} \leq K_{10}\left\|\left|D \chi_{k}\right|\right\|_{C\left(\bar{I}_{1} ; L^{2}\left(I_{2}\right)\right)}\left\|D \chi_{\ell} \mid\right\|_{C\left(\bar{I}_{1} ; L^{2}\left(I_{2}\right)\right)},
$$

where $K_{10}$ depends only on $\Omega$ and a bound for $\kappa_{i j}$ and $V$ in the norms of spaces in conditions (5.3).

The sesquilinear form $\mathcal{L}_{I_{1} h}^{N}(\cdot, \cdot)$ is Hermitian symmetric and has the following positive definiteness properties:

$$
\begin{gathered}
\frac{\nu \nu_{0 N}}{X_{1} \bar{\rho}_{0}} \max _{\bar{\omega}_{h}}\left|\mathbf{c}_{h}\right|^{2} \leq \mathcal{L}_{I_{1} h}^{N}\left(\mathbf{c}_{h}, \mathbf{c}_{h}\right) \quad \text { for any } \mathbf{c}_{h} \in H_{h}^{N}, \\
\nu K_{8, N}^{-1}\left\|\left|\delta_{1} \mathbf{c}_{h}\right|\right\|_{L_{h}^{2}\left(I_{1}\right)}^{2} \leq \mathcal{L}_{I_{1} h}^{N}\left(\mathbf{c}_{h}, \mathbf{c}_{h}\right) \quad \text { for any } \mathbf{c}_{h} \in H_{h}^{N} ;
\end{gathered}
$$

in the latter one, the regularity conditions (3.32) on $\kappa_{0}$ and $V_{0}$ are assumed to be valid.

Proof. Identity (5.6) is proved as (3.11) taking into account the equalities

$$
\left(D_{1} \eta\right)\left(x_{1 m-1 / 2}\right)=\delta_{1} \eta_{m-1 / 2}, \quad \eta\left(x_{1 m-1 / 2}\right)=s_{1} \eta_{m-1 / 2} \text { on } \omega_{h}^{*}, \text { for } \eta \in S_{h}\left(\bar{I}_{1}\right) .
$$

Therefore, for example

$$
\begin{aligned}
& \left(D_{1}\left(c_{h \ell} \chi_{\ell}\right)\right)\left(x_{1 m-1 / 2}, x_{2}\right) \\
= & \left(\delta_{1} c_{h \ell}\right)_{m-1 / 2} \chi_{\ell}\left(x_{1 m-1 / 2}, x_{2}\right)+\left(s_{1} c_{h \ell}\right)_{m-1 / 2}\left(D_{1} \chi_{\ell}\right)\left(x_{1 m-1 / 2}, x_{2}\right), \\
& \left(D_{2}\left(c_{h \ell} \chi_{\ell}\right)\right)\left(x_{1 m-1 / 2}, x_{2}\right)=\left(s_{1} c_{h \ell}\right)_{m-1 / 2}\left(D_{2} \chi_{\ell}\right)\left(x_{1 m-1 / 2}, x_{2}\right) .
\end{aligned}
$$

Bound (5.7) is proved similarly to (3.15).

Inequalities (5.8) and (5.9) are counterparts of (3.22) and (3.35) and are proved in a similar way. In fact, relations (5.6) and (5.4) imply

$$
\mathcal{L}_{I_{1} h}^{N}\left(\mathbf{c}_{h}, \mathbf{c}_{h}\right)=\mathcal{L}_{\Omega h}(v, v) \geq \nu\|\||D v|\left\|_{L^{2}\left(I_{2}\right)}\right\|_{L_{h}^{2}\left(I_{1}\right)}^{2} .
$$

Furthermore, we have

$$
\begin{aligned}
X_{1}\|\| \delta_{1} v\left\|_{L^{2}\left(I_{2}\right)}\right\|_{L_{h}^{2}\left(I_{1}\right)}^{2} & \geq \int_{I_{2}}\left|v\left(x_{1 m}, x_{2}\right)\right|^{2} d x_{2} \geq \frac{1}{\bar{\rho}_{0}} \int_{I_{2}}\left(\rho_{0}|v|^{2}\right)\left(x_{1 m}, x_{2}\right) d x_{2} \\
& =\frac{1}{\bar{\rho}_{0}}\left(G_{0}\left(x_{1 m}\right) \mathbf{c}_{h m}, \mathbf{c}_{h m}\right)_{\mathbb{C}^{N}} \text { on } \bar{\omega}_{h} .
\end{aligned}
$$


Applying the first equality (5.10) and property (3.3), we derive (5.8) from (5.13).

Next, exploiting the mesh equalities (5.11) and (5.12), we can get, similarly to (3.36), for $0<\gamma<1$

$$
\begin{aligned}
& \sum_{m=1}^{\bar{m}} \int_{I_{2}}|D v|^{2}\left(x_{1 m-1 / 2}, x_{2}\right) d x_{2} h_{m} \\
\geq & \frac{1-\gamma}{\bar{\rho}_{0}} \sum_{m=1}^{\bar{m}}\left(G^{(0)} \delta_{1} \mathbf{c}_{h}, \delta_{1} \mathbf{c}_{h}\right)_{\mathbb{C}^{N}}\left(x_{1 m-1 / 2}\right) h_{m} \\
& -\left(\frac{1}{\gamma}-1\right) \frac{1}{\bar{\rho}_{0}} \sum_{m=1}^{\bar{m}} \int_{I_{2}}\left(\left|\left(s_{1} \mathbf{c}_{h}, D_{1} \chi^{*}\right)_{\mathbb{C}^{N}}\right|^{2} \rho_{0}\right)\left(x_{1 m-1 / 2}, x_{2}\right) d x_{2} h_{m} \\
& +\frac{1}{K_{6}} \sum_{m=1}^{\bar{m}}\left(G^{(1)} s_{1} \mathbf{c}_{h}, s_{1} \mathbf{c}_{h}\right)_{\mathbb{C}^{N}}\left(x_{1 m-1 / 2}\right) h_{m} .
\end{aligned}
$$

The proof of (5.9) is completed as in the proof of Proposition 3.17 (taking into account the continuity of $G^{(1)}$ and $G^{(1)^{-1}}$ on $\bar{I}_{1}$ and the property $\left.D_{1} \chi \in C\left(\bar{I}_{1} ; L^{2}\left(I_{2}\right)\right)\right)$.

Proposition 5.2. The approximate Galerkin identity (5.2) means that the vector function $\mathbf{c}_{h}=\mathbf{c}_{h}^{(N)}$ of the coefficients in expansion (5.1) of the approximate solution $v_{h}^{(N)}$ satisfies the following finite difference scheme for the Galerkin BVP (3.24), (3.25)

$$
\begin{aligned}
& -\delta_{1}^{*}\left(A \delta_{1} \mathbf{c}_{h}^{(N)}+A^{(0)} s_{1} \mathbf{c}_{h}^{(N)}\right)+s_{1}^{*}\left(A^{(0)^{*}} \delta_{1} \mathbf{c}_{h}^{(N)}\right)+s_{1}^{*}\left(B s_{1} \mathbf{c}_{h}^{(N)}\right) \\
& \quad=s_{1}^{*} \mathbf{f}_{h}^{(N)} \text { on }\left\{x_{1 m}\right\}_{m=1}^{\bar{m}-1}, \\
& \left.\mathbf{c}_{h}^{(N)}\right|_{m=0, \bar{m}}=0
\end{aligned}
$$

where $\mathbf{f}_{h m-1 / 2}^{(N)}$ are the values of the vector function $\mathbf{f}^{(N)}$ introduced in Proposition 3.12.

Proof. The result follows from identities (5.2) and (5.6) by applying the elementary mesh identities

$$
\begin{aligned}
\sum_{m=1}^{\bar{m}} y_{m-1 / 2} \delta_{1} \eta_{m-1 / 2} h_{m} & =-\sum_{m=1}^{\bar{m}-1} \delta_{1}^{*} y_{m} \cdot \eta_{m} h_{m-1 / 2}, \\
\sum_{m=1}^{\bar{m}} y_{m-1 / 2} s_{1} \eta_{m-1 / 2} h_{m} & =\sum_{m=1}^{\bar{m}-1} s_{1}^{*} y_{m} \cdot \eta_{m} h_{m-1 / 2}
\end{aligned}
$$

for any $\eta \in H_{h}$ and any $y$ defined on $\omega_{h}^{*}$.

REMARK 5.3. Taking into account the elementary formula

$$
\delta_{1}^{*}\left(A^{(0)} s_{1} \mathbf{c}_{h}\right)=s_{1}^{*}\left(A^{(0)} \delta_{1} \mathbf{c}_{h}\right)+\delta_{1}^{*} A^{(0)} \cdot \mathbf{c}_{h},
$$

one can rewrite equation (5.14) in the form

$$
-\delta_{1}^{*}\left(A \delta_{1} \mathbf{c}_{h}\right)-2 \imath s_{1}^{*}\left(\tilde{A}^{(0)} \delta_{1} \mathbf{c}_{h}\right)+s_{1}^{*}\left(B s_{1} \mathbf{c}_{h}\right)-\delta_{1}^{*} A^{(0)} \cdot \mathbf{c}_{h}=s_{1}^{*} \mathbf{f}_{h}^{(N)}
$$


compare with (3.28).

Note that elements of the technique were used for example in [25].

Clearly the simplest method with linear elements considered here is unable to ensure high-order accuracy, and higher-order finite element methods in $x_{1}$ need to be invoked to this end.

\section{A 2D elliptic eigenvalue problem and its semi-discrete Galerkin ap- proximation}

In this section, we turn to an eigenvalue problem corresponding to the BVP (2.1), $(2.2)$

$$
\begin{aligned}
& \mathcal{H} \Psi=\lambda \rho \Psi \text { on } \Omega, \\
& \left.\Psi\right|_{\partial \Omega}=0,
\end{aligned}
$$

where the real weight function $\rho$ satisfies conditions $\rho \in L^{\infty}(\Omega)$ and $0<\rho \leq \rho(x)$ in $\Omega$. Let $\bar{\rho}:=\|\rho\|_{L^{\infty}(\Omega)}$.

We briefly recall its main properties. In its general form, this eigenvalue problem consists in finding the eigenfunctions $\Psi \in H_{0}^{1}(\Omega), \Psi \neq \equiv$, and the corresponding eigenvalues $\lambda$ satisfying the integral identity

$$
\mathcal{L}_{\Omega}(\Psi, \varphi)=\lambda \int_{\Omega} \Psi \varphi^{*} \rho d x \text { for any } \varphi \in H_{0}^{1}(\Omega)
$$

It is well known (for example see [13]) that all the eigenvalues are real and can be labeled, taking into account their multiplicity (i.e. the number of corresponding linearly independent eigenfunctions), in nondecreasing order

$$
0<\lambda_{1} \leq \lambda_{2} \leq \cdots \leq \lambda_{\ell} \leq \ldots, \quad \lambda_{\ell} \rightarrow \infty \quad \text { as } \ell \rightarrow \infty .
$$

The corresponding eigenfunctions $\left\{\Psi_{\ell}\right\}_{\ell=1}^{\infty}$ can be chosen to be real functions and to form an orthonormal basis in $L^{2}(\Omega)$ with the weight $\rho$, i.e.,

$$
\int_{\Omega} \Psi_{k} \Psi_{\ell} \rho d x=\delta_{k \ell} \text { for any } k, \ell
$$

and for any $w \in L^{2}(\Omega)$, the following expansion with respect to $\left\{\Psi_{\ell}\right\}_{\ell=1}^{\infty}$

$$
w(x)=\sum_{m=1}^{\infty} \widehat{w}_{m} \Psi_{m}(x), \text { with } \widehat{w}_{m}:=\int_{\Omega} w \Psi_{m} \rho d x,
$$

converges in $L^{2}(\Omega)$. Moreover, the system $\left\{\Psi_{\ell}\right\}_{\ell=1}^{\infty}$ has a second orthogonality property

$$
\mathcal{L}_{\Omega}\left(\Psi_{k}, \Psi_{\ell}\right)=\lambda_{k} \delta_{k \ell} \text { for any } k, \ell,
$$

and forms a basis in $H_{0}^{1}(\Omega)$ (i.e., for any $w \in H_{0}^{1}(\Omega)$, expansion (6.4) converges in $H_{0}^{1}(\Omega)$ as well).

Under the regularity conditions (2.8), the eigenfunctions are in fact strong solutions of the problem such that

$$
\Psi_{\ell} \in H^{2} \cap H_{0}^{1}(\Omega), \quad \mathcal{H} \Psi_{\ell}=\lambda_{\ell} \rho \Psi_{\ell} \text { in } L^{2}(\Omega) \text {, for any } \ell \geq 1 .
$$


Moreover, for any $w \in H^{2} \cap H_{0}^{1}(\Omega)$, expansion (6.4) converges in $H^{2}(\Omega)$ as well.

We finally recall the Courant-Fischer minmax principle

$$
\lambda_{\ell}=\min _{L_{\ell} \subset H_{0}^{1}(\Omega)} \max _{w \in L_{\ell}} \mathcal{R}_{\Omega}(w) \text { for } \ell \geq 1, \text { with } \mathcal{R}_{\Omega}(w):=\frac{\mathcal{L}_{\Omega}(w, w)}{\int_{\Omega} w^{2} \rho d x} .
$$

$\mathcal{R}_{\Omega}(w)$ is the Rayleigh quotient for the eigenvalue problem (6.1), (6.2), and the minimum is taken over all the $\ell$-dimensional subspaces $L_{\ell}$ in real $H_{0}^{1}(\Omega)$.

Following section 2, we seek approximate eigenfunctions $\Psi^{(N)} \in S_{N}, \Psi^{(N)} \not \equiv 0$, and eigenvalues $\lambda^{(N)}$ such that the integral identity

$$
\mathcal{L}_{\Omega}\left(\Psi^{(N)}, \varphi\right)=\lambda^{(N)} \int_{\Omega} \Psi^{(N)} \varphi^{*} \rho d x \text { for any } \varphi \in S_{N}
$$

is valid. Clearly this Galerkin identity appears by substituting $\Psi^{(N)}$ to $v^{(N)}$ and $\lambda^{(N)} \Psi^{(N)} \rho$ to $f$ in the previous Galerkin identity (2.17). Quite similarly, other equivalent forms (2.16), (2.18)-(2.20) imply the equivalent corresponding forms of (6.6); in particular, (2.20) leads to the orthogonality relations

$$
\int_{I_{2}}\left(\mathcal{H} \Psi^{(N)}-\lambda^{(N)} \rho \Psi^{(N)}\right)\left(x_{1}, x_{2}\right) \chi_{k}^{*}\left(x_{1}, x_{2}\right) d x_{2}=0 \text { for a.a. } x_{1} \in I_{1},
$$

with $k=1, \ldots, N$.

Proposition 6.1. Let $\mathbf{c}^{(N)}$ be the vector-valued function of coefficients in the expansion

$$
\Psi^{(N)}\left(x_{1}, x_{2}\right)=\sum_{\ell=1}^{N} c_{\ell}\left(x_{1}\right) \chi_{\ell}\left(x_{1}, x_{2}\right) \quad \text { on } \bar{\Omega}
$$

of an approximate eigenfunction $\Psi^{(N)}$. Then the Galerkin identity (6.6) means that $\mathbf{c}^{(N)} \in\left[H_{0}^{1}\left(I_{1}\right)\right]^{N}, \mathbf{c}^{(N)} \not \equiv \mathbf{0}$, together with $\lambda^{(N)}$ satisfy the integral identity

$$
\mathcal{L}_{I_{1}}^{N}\left(\mathbf{c}^{(N)}, \mathbf{d}\right)=\lambda^{(N)} \int_{I_{1}}\left(M \mathbf{c}^{(N)}, \mathbf{d}\right)_{\mathbb{C}^{N}} d x_{1} \text { for any } \mathbf{d} \in\left[H_{0}^{1}\left(I_{1}\right)\right]^{N},
$$

i.e., they satisfy in a weak sense the eigenvalue problem for the system of ODEs corresponding to the Galerkin BVP (3.24), (3.25)

$$
\begin{aligned}
& \mathcal{H}_{N} \mathbf{c}^{(N)} \equiv-D_{1}\left(A D_{1} \mathbf{c}^{(N)}+A^{(0)} \mathbf{c}^{(N)}\right)+A^{(0)^{*}} D_{1} \mathbf{c}^{(N)}+B \mathbf{c}^{(N)}=\lambda^{(N)} M \mathbf{c}^{(N)},(6.9) \\
& \left.\mathbf{c}^{(N)}\right|_{x_{1}=0, X_{1}}=0
\end{aligned}
$$

where the $N \times N$ mass matrix $M$ has entries, for any $1 \leq k \leq N$ and $1 \leq \ell \leq N$

$$
m_{k \ell}\left(x_{1}\right)=\int_{I_{2}}\left(\chi_{k}^{*} \chi_{\ell} \rho\right)\left(x_{1}, x_{2}\right) d x_{2} \text { on } I_{1} .
$$

Proof. The result immediately follows from Lemma 3.7 together with the identity

$$
\int_{I_{1}} \Psi^{(N)} \varphi^{*} \rho d x_{1}=\int_{I_{1}}\left(M \mathbf{c}^{(N)}, \mathbf{d}\right)_{\mathbb{C}^{N}} d x_{1} \text { for any } \varphi \in S_{N},
$$


where $\varphi$ and $\mathbf{d}$ are related through (3.10).

Remark 6.2. Comparing formulas (3.12) and (6.11) and exploiting inequalities (2.10) and (3.18), we get the following bound and the positive-definiteness property:

$$
\begin{gathered}
\left\|m_{k \ell}\right\|_{L^{\infty}\left(I_{1}\right)} \leq c(\Omega) \bar{\rho}\left\|\chi_{k}\right\|_{H^{1,0}(\Omega)}\left\|\chi_{\ell}\right\|_{H^{1,0}(\Omega)} \text { for any } k, \ell, \\
\frac{\rho \nu_{0 N}}{\bar{\rho}_{0}} \mathbb{I} \leq \frac{\rho}{\bar{\rho}_{0}} G_{0}\left(x_{1}\right) \leq \frac{1}{\left\|\left(\rho_{0} / \rho\right)\left(x_{1}, \cdot\right)\right\|_{L^{\infty}\left(I_{2}\right)}} G_{0}\left(x_{1}\right) \leq\left(M\left(x_{1}\right) \mathbf{c}, \mathbf{c}\right)_{\mathbb{C}^{N}},
\end{gathered}
$$

for any $\mathbf{c} \in \mathbb{C}^{N}$ and a.a. $x_{1} \in I_{1}$.

Proposition 6.3. The eigenvalues of the approximate eigenvalue problem (6.6) are real and can be labeled in the nondecreasing order taking into account their multiplicity

$$
0<\lambda_{1}^{(N)} \leq \lambda_{2}^{(N)} \leq \cdots \leq \lambda_{\ell}^{(N)} \leq \ldots, \quad \lambda_{\ell}^{(N)} \rightarrow \infty \quad \text { as } \ell \rightarrow \infty .
$$

The corresponding eigenfunctions $\left\{\Psi_{\ell}^{(N)}\right\}_{\ell=1}^{\infty}$ can be chosen to form an orthonormal basis in $S_{N}^{0}$ considered as a subspace of $L^{2}(\Omega)$ with the weight $\rho$, i.e.

$$
\int_{\Omega} \Psi_{k}^{(N)} \Psi_{\ell}^{(N)} \rho d x=\delta_{k \ell} \text { for any } k, \ell
$$

and, for any $v \in S_{N}^{0}$, the Fourier expansion with respect to the system $\left\{\Psi_{m}^{(N)}\right\}_{m=1}^{\infty}$

$$
v(x)=\sum_{m=1}^{\infty} \hat{v}_{m}^{(N)} \Psi_{m}^{(N)}(x) \quad \text { on } \Omega, \quad \text { with } \hat{v}_{m}^{(N)}=\int_{\Omega} v \Psi_{m}^{(N)} \rho d x
$$

converges in $L^{2}(\Omega)$. Moreover, the system $\left\{\Psi_{\ell}^{(N)}\right\}_{\ell=1}^{\infty}$ has a second orthogonality property

$$
\mathcal{L}_{\Omega}\left(\Psi_{k}^{(N)}, \Psi_{\ell}^{(N)}\right)=\lambda_{\ell}^{(N)} \delta_{k \ell} \text { for any } k, \ell
$$

and form a basis in $S_{N}$ considered as a subspace of $H_{0}^{1}(\Omega)$, i.e., for any $v \in S_{N}$, expansion (6.13) converges in $H_{0}^{1}(\Omega)$.

Under the regularity conditions (2.8) and (3.16), the eigenfunctions are actually strong solutions of the problem such that $\Psi_{\ell}^{(N)} \in H^{2} \cap H_{0}^{1}(\Omega)$ and the corresponding equation (6.9) is satisfied in $\left[L^{2}\left(I_{1}\right)\right]^{N}$, for any $\ell \geq 1$.

In addition, the Courant-Fischer minmax principle holds:

$$
\lambda_{\ell}^{(N)}=\min _{L_{\ell} \subset S_{N}} \max _{w \in L_{\ell}} \mathcal{R}_{\Omega}(w) \text { for } \ell \geq 1
$$

Proof. The results follow from Propositions 3.12, 3.19 and 6.1 and from the general theory of self-adjoint elliptic operators (covering the case of operators of self-adjoint systems of ODEs as well); in particular see [13]. Identities (3.11) and (6.12) are also essential in order to translate the results from problem $(6.9),(6.10)$ back to (6.6). $\square$ 
COROLlary 6.4. The one-sided approximation property $\lambda_{\ell} \leq \lambda_{\ell}^{(N)}$ holds for any $\ell \geq 1$.

The result is well known for the Rayleigh-Ritz method and follows directly from the Courant-Fischer minmax principles (6.5) and (6.14).

We complete this section by the error bounds in the case of the specific basis functions studied in section 4 . For this purpose, we need to introduce a family of Hilbert spaces associated to the eigenvalue problem (6.1), (6.2)

$$
\mathbf{H}^{\theta}(\Omega):=\left\{w \in L^{2}(\Omega) ;\|w\|_{\mathbf{H}^{\theta}(\Omega)}:=\left[\sum_{m=1}^{\infty} \lambda_{m}^{\theta}\left|\widehat{w}_{m}\right|^{2}\right]^{1 / 2}<\infty\right\}, \text { for } \theta \geq 0,
$$

with the coefficients $\widehat{w}_{m}$ given in (6.4). Clearly, the spaces are similar to those introduced in section 4 . In particular, we have

$$
\|w\|_{\mathbf{H}^{0}(\Omega)}=\|\sqrt{\rho} w\|_{L^{2}(\Omega)}, \quad\|w\|_{\mathbf{H}^{1}(\Omega)}=\|w\|_{\mathcal{E}(\Omega)},
$$

so that $\mathbf{H}^{0}(\Omega)=L^{2}(\Omega)$ and $\mathbf{H}^{1}(\Omega)=H_{0}^{1}(\Omega)$, up to an equivalence of norms. Under the regularity conditions $(2.8), \mathbf{H}^{2}(\Omega)=H^{2} \cap H_{0}^{1}(\Omega)$ in a similar sense and $\|w\|_{\mathbf{H}^{2}(\Omega)}=$ $\left\|\frac{1}{\sqrt{\rho}} \mathcal{H} w\right\|_{L^{2}(\Omega)}$. Moreover, for $\theta>2$ the space $\mathbf{H}^{\theta}(\Omega)$ consists of functions $w \in H_{0}^{1}(\Omega)$ such that $\rho^{-1} \mathcal{H} w \in \mathbf{H}^{\theta-2}(\Omega)$ and $\|w\|_{\mathbf{H}^{\theta}(\Omega)}=\left\|\rho^{-1} \mathcal{H} w\right\|_{\mathbf{H}^{\theta-2}(\Omega)}$.

Below, we will need the following embedding inequality:

$$
\|w\|_{\mathbf{H}^{0, \theta}(\Omega)}+\left\|D_{1} w\right\|_{\mathbf{H}^{0, \theta-1}(\Omega)} \leq C_{\theta}\|w\|_{\mathbf{H}^{\theta}(\Omega)} \text { for any } w \in \mathbf{H}^{\theta}(\Omega),
$$

with some $\theta \geq 1$. Notice that this is valid for $\theta=1$ and, under the regularity conditions (2.8) and $D_{2} \kappa_{0}, V_{0} \in \tilde{L}^{2, \infty}(\Omega)$, for $\theta=2$ as well. According to the interpolation space theory [3], if the inequality is valid for some $\theta=\theta_{0}, \theta_{1}$ with $1 \leq \theta_{0}<\theta_{1}$, it is valid for any $\theta_{0} \leq \theta \leq \theta_{1}$.

Let $\mathcal{P}_{N}^{(1)}: H_{0}^{1}(\Omega) \rightarrow S_{N}$ be another (different from $\mathcal{P}_{N}$ ) projector such that

$$
\mathcal{L}_{\Omega}\left(w-\mathcal{P}_{N}^{(1)} w, \varphi\right)=0 \text { for any } \varphi \in S_{N} .
$$

Proposition 6.5. Let the regularity conditions (2.8), (4.20), (4.46) together with (4.33) be valid. Let $\theta>1$ and inequality (6.15) together with (for $\theta>3 / 2$ ) (4.42) for $\beta=\theta-5 / 2$ be valid as well. Then the following error bound for the approximate eigenvalues hold:

$$
0 \leq \lambda_{\ell}^{(N)}-\lambda_{\ell} \leq K \lambda_{\ell}^{\tilde{\theta}}(N+1)^{-2(\theta-1)}(1+\log N),
$$

for any $\ell \geq 1$ and sufficiently large $N \geq N_{0}\left(\lambda_{\ell}, \theta\right)$, where $K$ does not depend on $\ell$ and

$$
\tilde{\theta}:= \begin{cases}\theta & \text { for } \theta \geq 2, \\ \theta / 2+1 & \text { for } \theta<2 .\end{cases}
$$

Proof. We apply the technique described in [23], section 6.3 which allows to reduce the study to the case of the BVP. Let $E_{\ell}$ be the (real) linear hull of $\left\{\Psi_{m}\right\}_{m=1}^{\ell}$. We set

$$
\sigma_{\ell}^{(N)}:=\max _{w \in E_{\ell},\|\sqrt{\rho} w\|_{\Omega}=1}\left|\int_{\Omega}\left[w^{2}-\left(\mathcal{P}_{N}^{(1)} w\right)^{2}\right] \rho d x\right|
$$


Then, provided that $\sigma_{\ell}^{(N)}<1$, the following upper bound holds:

$$
\lambda_{\ell}^{(N)} \leq \frac{\lambda_{\ell}}{1-\sigma_{\ell}^{(N)}}
$$

see [23], Lemma 6.1 (notice that the bound is a consequence of formulas (6.5) and (6.14)). Moreover, if $w \in E_{\ell}$ and $\|\sqrt{\rho} w\|_{\Omega}=1$, the following formula holds

$$
\int_{\Omega} w\left(w-\mathcal{P}_{N}^{(1)} w\right) \rho d x=\mathcal{L}_{\Omega}\left(W-\mathcal{P}_{N}^{(1)} W, w-\mathcal{P}_{N}^{(1)} w\right)
$$

where $W \in E_{\ell}$ is such that $\mathcal{H} W=\rho w$ in $\Omega$ (see [23], Lemma 6.2). Clearly $\hat{W}_{m}=\hat{w}_{m} / \lambda_{m}$ for any $m$, thus

$$
\begin{gathered}
\|W\|_{\mathbf{H}^{\theta}(\Omega)} \leq \lambda_{\ell}^{\theta / 2-1}\|W\|_{\mathbf{H}^{2}(\Omega)}=\lambda_{\ell}^{\theta / 2-1}\|w\|_{\mathbf{H}^{0}(\Omega)}=\lambda_{\ell}^{\theta / 2-1} \text { for } \theta \geq 2 \\
\|W\|_{\mathbf{H}^{\theta}(\Omega)} \leq \lambda_{1}^{-(1-\theta / 2)}\|W\|_{\mathbf{H}^{2}(\Omega)}=\lambda_{1}^{-(1-\theta / 2)} \quad \text { for } 0 \leq \theta \leq 2
\end{gathered}
$$

Due to Corollary 6.4 and bound (6.18), it is sufficient to bound $\sigma_{\ell}^{(N)}$ starting from the obvious formula

$$
w^{2}-\left(\mathcal{P}_{N}^{(1)} w\right)^{2}=2 w\left(w-\mathcal{P}_{N}^{(1)} w\right)-\left(w-\mathcal{P}_{N}^{(1)} w\right)^{2}
$$

For $w \in E_{\ell}$ with $\|\sqrt{\rho} w\|_{\Omega}=1$ and $\theta>1$, sequentially applying formula (6.19), the definition of $\mathcal{P}_{N}^{(1)}$ and Proposition 4.14, we first have

$$
\begin{aligned}
& \left|\int_{\Omega} w\left(w-\mathcal{P}_{N}^{(1)} w\right) \rho d x\right| \\
\leq & \left\|W-\mathcal{P}_{N}^{(1)} W\right\|_{\mathcal{E}(\Omega)}\left\|w-\mathcal{P}_{N}^{(1)} w\right\|_{\mathcal{E}(\Omega)} \\
\leq & \left\|W-\mathcal{P}_{N} W\right\|_{\mathcal{E}(\Omega)}\left\|w-\mathcal{P}_{N} w\right\|_{\mathcal{E}(\Omega)} \\
\leq & {\left[K_{1} K_{17}(N+1)^{-(\theta-1)}\left(1+\log ^{1 / 2} N\right)\right]^{2}\left(\|W\|_{\mathbf{H}^{0, \theta}(\Omega)}+\left\|D_{1} W\right\|_{\mathbf{H}^{0, \theta-1}(\Omega)}\right) } \\
& \times\left(\|w\|_{\mathbf{H}^{0, \theta}(\Omega)}+\left\|D_{1} w\right\|_{\mathbf{H}^{0, \theta-1}(\Omega)}\right) .
\end{aligned}
$$

By virtue of embedding (6.15) and estimates (6.20), (6.21) and

$$
\|w\|_{\mathbf{H}^{\theta}(\Omega)} \leq \lambda_{\ell}^{\theta / 2}\|w\|_{\mathbf{H}^{0}(\Omega)}=\lambda_{\ell}^{\theta / 2}
$$

we have

$$
\left|\int_{\Omega} w\left(w-\mathcal{P}_{N}^{(1)} w\right) \rho d x\right| \leq 2\left(C_{\theta} K_{1} K_{17}\right)^{2} \lambda_{1}^{-(\tilde{\theta}-\theta)} \lambda_{\ell}^{\tilde{\theta}-1}(N+1)^{-2(\theta-1)}(1+\log N) .
$$

Next, the $L^{2}(\Omega)$-bound (4.47) together with, once again, (6.15) and (6.22) imply that

$$
\begin{aligned}
\int_{\Omega}\left(w-\mathcal{P}_{N}^{(1)} w\right)^{2} \rho d x & \leq \bar{\rho} K_{19}^{2}(N+1)^{-2 \theta}(1+\log N)^{2}\left(\|w\|_{\mathbf{H}^{0, \theta}(\Omega)}+\left\|D_{1} w\right\|_{\mathbf{H}^{0, \theta}(\Omega)}\right)^{2} \\
& \leq \bar{\rho}\left(C_{\theta} K_{19}\right)^{2} \lambda_{\ell}^{\theta}(N+1)^{-2 \theta}(1+\log N)^{2}
\end{aligned}
$$


Summarizing all of these relations, we obtain

$$
\begin{gathered}
\left|\sigma_{\ell}^{(N)}\right| \leq C_{\theta}^{2}\left[\left(2 K_{1} K_{17}\right)^{2} \lambda_{1}^{-(\tilde{\theta}-\theta)}+\bar{\rho} K_{19}^{2} \lambda_{\ell}^{\theta-\tilde{\theta}+1}(N+1)^{-2}(1+\log N)\right] \\
\times \lambda_{\ell}^{\tilde{\theta}-1}(N+1)^{-2(\theta-1)}(1+\log N) .
\end{gathered}
$$

For sufficiently large $N \geq N_{0}\left(\lambda_{\ell}, \theta\right)$, we finally obtain

$$
\left|\sigma_{\ell}^{(N)}\right| \leq K \lambda_{\ell}^{\tilde{\theta}-1}(N+1)^{-2(\theta-1)}(1+\log N) \leq \frac{1}{2}
$$

with $K$ independent of $\ell$. The upper bound (6.18) now gives that

$$
\lambda_{\ell}^{(N)} \leq \lambda_{\ell}\left(1+2\left|\sigma_{\ell}^{(N)}\right|\right)=\lambda_{\ell}+2 \lambda_{\ell}\left|\sigma_{\ell}^{(N)}\right|,
$$

which completes the proof.

We omit any explicit expression for $N_{0}\left(\lambda_{\ell}, \theta\right)$ and note only that it increases with respect to $\lambda_{\ell}$.

In order to state error bounds for the approximate eigenfunctions, we recall that, for simple $\lambda_{\ell}$, the eigenfunctions $\Psi_{\ell}$ are defined up to the multiplier \pm 1 . For such a $\lambda_{\ell}$, let

$$
r_{\ell}:=\min _{m \neq \ell}\left|\frac{\lambda_{m}}{\lambda_{\ell}}-1\right|=\left\{\begin{array}{l}
\min \left\{1-\frac{\lambda_{\ell-1}}{\lambda_{\ell}}, \frac{\lambda_{\ell+1}}{\lambda_{\ell}}-1\right\} \text { for } \ell \geq 2, \\
\frac{\lambda_{2}}{\lambda_{1}}-1 \text { for } \ell=1,
\end{array}\right.
$$

be the relative distance from $\lambda_{\ell}$ to other eigenvalues.

Proposition 6.6. Let the hypotheses of Proposition 6.5 be valid. Then for any $\ell \geq 1$ such that $\lambda_{\ell}$ is simple, the following error bounds for the approximate eigenfunctions hold

$$
\begin{gathered}
\left\|\Phi_{\ell}-\Psi_{\ell}^{(N)}\right\|_{L^{2}(\Omega)} \leq K \lambda_{\ell}^{\theta / 2}(N+1)^{-\theta}(1+\log N), \\
\left\|\Phi_{\ell}-\Psi_{\ell}^{(N)}\right\|_{H^{1}(\Omega)} \leq K \lambda_{\ell}^{\tilde{\theta} / 2}(N+1)^{-(\theta-1)}\left(1+\log ^{1 / 2} N\right),
\end{gathered}
$$

where either $\Phi_{\ell}=\Psi_{\ell}$ or $\Phi_{\ell}=-\Psi_{\ell}$, for sufficiently large $N \geq N_{1}\left(\lambda_{\ell-1}, \lambda_{\ell}, \theta\right)\left(\lambda_{\ell-1}\right.$ disappears for $\ell=1$ ).

The multipliers $K$ depend on $r_{\ell}$ but not directly on $\ell$.

Proof. According to [23], section 6.3, the following relations hold for the exact and approximate $\ell$ th eigenfunctions

$$
\begin{gathered}
\left\|\Phi_{\ell}-\Psi_{\ell}^{(N)}\right\|_{\mathcal{E}(\Omega)}^{2}=\lambda_{\ell}\left\|\sqrt{\rho}\left(\Phi_{\ell}-\Psi_{\ell}^{(N)}\right)\right\|_{\Omega}^{2}+\lambda_{\ell}^{(N)}-\lambda_{\ell}, \\
\left\|\sqrt{\rho}\left(\Phi_{\ell}-\Psi_{\ell}^{(N)}\right)\right\|_{\Omega} \leq 2\left[1+\left(r_{\ell}^{(N)}\right)^{-1}\right]\left\|\sqrt{\rho}\left(\Phi_{\ell}-\mathcal{P}_{N}^{(1)} \Phi_{\ell}\right)\right\|_{\Omega},
\end{gathered}
$$

with

$$
r_{\ell}^{(N)}:=\min _{m \neq \ell}\left|\frac{\lambda_{m}^{(N)}}{\lambda_{\ell}}-1\right| .
$$


Inequality (6.29) assumes that $\lambda_{\ell}$ is simple and that $\Phi_{\ell}= \pm \Psi_{\ell}$ is chosen such that $\int_{\Omega} \Phi_{\ell} \Psi_{\ell}^{(N)} \rho d x \geq 0$.

By Corollary 6.4 we have that

$$
r_{\ell}^{(N)} \geq\left\{\begin{array}{l}
\min \left\{\min _{1 \leq m \leq \ell-1}\left|\frac{\lambda_{m}^{(N)}}{\lambda_{\ell}}-1\right|, \frac{\lambda_{\ell+1}}{\lambda_{\ell}}-1\right\} \text { for } \ell \geq 2, \\
\frac{\lambda_{2}}{\lambda_{1}}-1 \text { for } \ell=1 .
\end{array}\right.
$$

Furthermore, for $\ell \geq 2$, we obtain

$$
\min _{1 \leq m \leq \ell-1}\left|\frac{\lambda_{m}^{(N)}}{\lambda_{\ell}}-1\right| \geq \frac{1}{2}\left(1-\frac{\lambda_{\ell-1}}{\lambda_{\ell}}\right),
$$

provided that $\lambda_{\ell-1}^{(N)}$ is so close to $\lambda_{\ell-1}$ that

$$
\lambda_{\ell-1}^{(N)} \leq \frac{\lambda_{\ell-1}+\lambda_{\ell}}{2}=\lambda_{\ell-1}\left[1+\frac{1}{2}\left(\frac{\lambda_{\ell}}{\lambda_{\ell-1}}-1\right)\right] .
$$

This inequality follows from (6.25) for sufficiently large $N \geq \tilde{N}_{0}\left(\lambda_{\ell-1}, \lambda_{\ell}, \theta\right)$ such that

$$
\sigma_{\ell-1}^{(N)} \leq \frac{1}{2} \min \left\{\frac{1}{2}\left(\frac{\lambda_{\ell}}{\lambda_{\ell-1}}-1\right), 1\right\}
$$

compare with (6.24). Consequently $r_{\ell}^{(N)} \geq r_{\ell} / 2$ for any $\ell \geq 1$ (this fills in a gap in the argument of [23]). After that, the $L^{2}(\Omega)$ error bound (6.26) is a consequence of (6.29) and (6.23), for $w=\Phi_{\ell}$.

Equality (6.28) together with the error bounds (6.26) and (6.17) imply that

$$
\begin{gathered}
\sqrt{\nu}\left\|\Phi_{\ell}-\Psi_{\ell}^{(N)}\right\|_{H_{0}^{1}(\Omega)} \leq \sqrt{\lambda_{\ell}}\left\|\sqrt{\rho}\left(\Phi_{\ell}-\Psi_{\ell}^{(N)}\right)\right\|_{\Omega}+\sqrt{\lambda_{\ell}^{(N)}-\lambda_{\ell}} \\
\leq K\left[\lambda_{\ell}^{(\theta-\tilde{\theta}+1) / 2}(N+1)^{-1}\left(1+\log ^{1 / 2} N\right)+1\right] \lambda_{\ell}^{\tilde{\theta} / 2}(N+1)^{-(\theta-1)}\left(1+\log ^{1 / 2} N\right),
\end{gathered}
$$

which leads to (6.27).

Remark 6.7. In Proposition 6.6, the assumption of simplicity of $\lambda_{\ell}$ is not essential and error bounds of the same orders are valid in the case of multiple $\lambda_{\ell}$ as well (according to [23]). The orders are also the same as in the case of the BVP in Corollary 4.15 .

Recall that in the case where $\lambda_{\ell-1}<\lambda_{\ell}=\cdots=\lambda_{\ell+p}<\lambda_{\ell+p+1}$ with $p \geq 1$, the eigenfunctions $\Psi_{\ell}, \ldots, \Psi_{\ell+p}$ are defined up to an arbitrary linear transformation, orthogonal with respect to the norm $\|\sqrt{\rho} w\|_{L^{2}(\Omega)}$.

Note that, for $\ell=1$, bound (6.26) holds for any $N \geq 1$.

7. An initial-boundary value problem for the time-dependent generalized Schrödinger equation and its semi-discrete Galerkin approximation

Finally we consider the generalized time-dependent Schrödinger equation

$$
\imath \rho D_{t} \psi=\mathcal{H} \psi \text { in } Q:=\Omega \times \mathbb{R}^{+},
$$


supplemented with the boundary and initial conditions

$$
\left.\psi\right|_{\partial \Omega \times \mathbb{R}^{+}}=0,\left.\quad \psi\right|_{t=0}=\psi^{0}(x) \text { on } \Omega .
$$

Recall that the operator $\mathcal{H}$ and the function $\rho$ have been introduced in equations (2.1) and (6.1).

By definition, a weak solution to the IBVP (7.1), (7.2) with the properties

$$
\psi \in C\left(\overline{\mathbb{R}}^{+} ; H_{0}^{1}(\Omega)\right), D_{t}(\rho \psi) \in C\left(\overline{\mathbb{R}}^{+} ; H^{-1}(\Omega)\right)
$$

satisfies the integral identity

$$
\imath\left\langle D_{t}(\rho \psi)(\cdot, t), \varphi(\cdot)\right\rangle_{\Omega}=\mathcal{L}_{\Omega}(\psi(\cdot, t), \varphi(\cdot)) \text { for any } \varphi \in H_{0}^{1}(\Omega) \text { and } t \geq 0,
$$

together with the initial condition $\left.\psi\right|_{t=0}=\psi^{0} \in H_{0}^{1}(\Omega)$.

This solution exists and is unique. Moreover, the following conservation laws and bound hold:

$$
\begin{aligned}
\|(\sqrt{\rho} \psi)(\cdot, t)\|_{L^{2}(\Omega)} & =\left\|\sqrt{\rho} \psi^{0}\right\|_{L^{2}(\Omega)} \text { for any } t \geq 0, \\
\|\psi(\cdot, t)\|_{\mathcal{E}(\Omega)} & =\left\|\psi^{0}\right\|_{\mathcal{E}(\Omega)} \text { for any } t \geq 0, \\
\sup _{t \geq 0}\left\|D_{t}(\rho \psi)(\cdot, t)\right\|_{H^{-1}(\Omega)} & \leq K_{1}\left\|\psi^{0}\right\|_{\mathcal{E}(\Omega)} .
\end{aligned}
$$

The solution can be Fourier-expanded as

$$
\psi(x, t)=\sum_{m=1}^{\infty} e^{-\imath \lambda_{m} t} \hat{\psi}_{m}^{0} \Psi_{m}(x), \text { with } \hat{\psi}_{m}^{0}=\int_{\Omega} \psi^{0} \Psi_{m} \rho d x
$$

with respect to the system of eigenfunctions of the corresponding eigenvalue problem (6.1), (6.2). The expansion converges in $H_{0}^{1}(\Omega)$ uniformly in $t \geq 0$.

Concerning bound (7.6), notice that, for any $t \geq 0$

$$
\left\|D_{t}(\rho \psi)(\cdot, t)\right\|_{H^{-1}(\Omega)}=\sup _{\varphi \in H_{0}^{1}(\Omega)} \frac{\left|\mathcal{L}_{\Omega}(\psi(\cdot, t), \varphi(\cdot))\right|}{\|\varphi\|_{H_{0}^{1}(\Omega)}} \leq K_{1}\|\psi(\cdot, t)\|_{\mathcal{E}(\Omega)},
$$

due to identity (7.3) and inequalities (2.6).

The proof of the existence and of the conservation laws are obtained by the Fourier method justifying expansion (7.7). The uniqueness can be proved using the energy method.

Our semi-discrete Galerkin method for the IBVP (7.1), (7.2) exploits an approximate solution $y^{(N)}(\cdot, t) \in S_{N}$ for any $t \geq 0$, more precisely, of the form

$$
y^{(N)}\left(x_{1}, x_{2}, t\right)=\sum_{\ell=1}^{N} c_{\ell}\left(x_{1}, t\right) \chi_{\ell}\left(x_{1}, x_{2}\right) \text { in } \bar{Q},
$$

with the vector-function of coefficients $\mathbf{c}$ such that

$$
\mathbf{c} \in C\left(\overline{\mathbb{R}}^{+} ;\left[H_{0}^{1}\left(I_{1}\right)\right]^{N}\right), \quad D_{t}(M \mathbf{c}) \in C\left(\overline{\mathbb{R}}^{+} ;\left[H^{-1}\left(I_{1}\right)\right]^{N}\right),
$$

where the matrix $M$ has been introduced in (6.11). We seek an approximate solution satisfying the integral identity

$$
\imath D_{t} \int_{\Omega}\left(\rho y^{(N)} \varphi^{*}\right)(x, t) d x=\mathcal{L}_{\Omega}\left(y^{(N)}(\cdot, t), \varphi(\cdot)\right) \text { for any } \varphi \in S_{N} \text { and } t \geq 0
$$


(compare with (7.3)), together with the initial condition

$$
\left.y^{(N)}\right|_{t=0}=y^{(N), 0}:=\sum_{\ell=1}^{N} c_{\ell}^{0} \chi_{\ell} \in S_{N}
$$

where $y^{(N), 0}$ is a given approximation of $\psi^{0}$, with a vector of coefficients $\mathbf{c}^{0}:=$ $\left(c_{1}^{0}, \ldots, c_{N}^{0}\right)^{T}$.

Proposition 7.1. Properties (7.9) imply that

$$
y^{(N)} \in C\left(\overline{\mathbb{R}}^{+} ; H_{0}^{1}(\Omega)\right), D_{t} \int_{\Omega} \rho y^{(N)} \varphi^{*} d x \in C\left(\overline{\mathbb{R}}^{+}\right) \text {for any } \varphi \in S_{N},
$$

so that identity (7.10) and the initial condition (7.11) are well-defined.

Moreover, the approximate Galerkin time-dependent problem (7.10), (7.11) is equivalent to an IBVP for the time-dependent Schrödinger-like system of $1 D$ (in space) equations

$$
\begin{aligned}
\imath D_{t}(M \mathbf{c}) & =\mathcal{H}_{N} \mathbf{c} \text { in } C\left(\overline{\mathbb{R}}^{+} ;\left[H^{-1}\left(I_{1}\right)\right]^{N}\right), \\
\left.\mathbf{c}\right|_{t=0} & =\mathbf{c}^{0} \in\left[H_{0}^{1}\left(I_{1}\right)\right]^{N},
\end{aligned}
$$

where the operator $\mathcal{H}_{N}$ has been introduced in equation (3.24).

REMARK 7.2. Equation (7.13) can be also rewritten in the form of integral identity

$$
\imath\left\langle D_{t}(M \mathbf{c})(\cdot, t), \mathbf{d}(\cdot)\right\rangle_{I_{1}}=\mathcal{L}_{I_{1}}^{N}(\mathbf{c}(\cdot, t), \mathbf{d}(\cdot)) \text { for any } \mathbf{d} \in\left[H_{0}^{1}\left(I_{1}\right)\right]^{N} \text { and } t \geq 0,
$$

with the sesquilinear form $\mathcal{L}_{I_{1}}^{N}(\cdot, \cdot)$ introduced in Lemma 3.7.

Proof. The first property (7.12) follows from inequality (2.15). The second one is a consequence of the identity

$$
D_{t} \int_{\Omega}\left(\rho y^{(N)} \varphi^{*}\right)(x, t) d x=\left\langle D_{t}(M \mathbf{c})(\cdot, t), \mathbf{d}(\cdot)\right\rangle_{I_{1}} \text { for any } \mathbf{d} \in\left[H_{0}^{1}\left(I_{1}\right)\right]^{N},
$$

where $\varphi$ and $\mathbf{d}$ are related by expansion (3.10) and that follows from identity (6.12).

Integral identity (7.10) can be rewritten as (7.15) due to (7.16) and (3.11). Finally, equation (7.13) is the operator form of (7.15) taking into account that $\mathcal{H}_{N}$ : $\left[H_{0}^{1}\left(I_{1}\right)\right]^{N} \rightarrow\left[H^{-1}\left(I_{1}\right)\right]^{N}$ is a bounded operator.

Proposition 7.3. The Galerkin IBVP (7.13), (7.14) has a unique solution in the class (7.9).

Moreover, the following conservation laws hold

$$
\begin{aligned}
\left\|\sqrt{\rho} y^{(N)}(\cdot, t)\right\|_{L^{2}(\Omega)} & =\left\|\sqrt{\rho} y^{(N), 0}\right\|_{L^{2}(\Omega)} \quad \text { for any } t \geq 0, \\
\left\|y^{(N)}(\cdot, t)\right\|_{\mathcal{E}(\Omega)} & =\left\|y^{(N), 0}\right\|_{\mathcal{E}(\Omega)} \quad \text { for } \text { any } t \geq 0 ;
\end{aligned}
$$

compare with (7.4), (7.5).

$y^{(N)}$ can be Fourier-expanded as

$$
y^{(N)}(x, t)=\sum_{m=1}^{\infty} e^{-\imath \lambda_{m}^{(N)} t} \hat{y}_{m}^{(N), 0} \Psi_{m}^{(N)}(x, t), \quad \text { with } \hat{y}_{m}^{(N), 0}:=\int_{\Omega} y_{m}^{(N), 0} \Psi_{m}^{(N)} \rho d x,
$$


with respect to the system of eigenfunctions of the Galerkin eigenvalue problem (6.6). The expansion converges in $H_{0}^{1}(\Omega)$ uniformly in $t \geq 0$.

Proof. The proof of the existence and of the conservation laws

$$
\begin{gathered}
\max _{t \geq 0} \int_{I_{1}}(M \mathbf{c}, \mathbf{c})_{\mathbb{C}^{N}}\left(x_{1}, t\right) d x_{1}=\int_{I_{1}}\left(M \mathbf{c}^{0}, \mathbf{c}^{0}\right)_{\mathbb{C}^{N}} d x_{1} \text { for any } t \geq 0, \\
\max _{t \geq 0} \mathcal{L}_{I_{1}}^{N}(\mathbf{c}(\cdot, t), \mathbf{c}(\cdot, t))=\mathcal{L}_{I_{1}}^{N}\left(\mathbf{c}^{0}, \mathbf{c}^{0}\right) \text { for any } t \geq 0
\end{gathered}
$$

together with a bound

$$
\sup _{t \geq 0}\left\|D_{t}(M \mathbf{c})(\cdot, t)\right\|_{\left[H^{-1}\left(I_{1}\right)\right]^{N}} \leq K_{N}\left\|\mathbf{c}^{0}\right\|_{\left[H_{0}^{1}\left(I_{1}\right)\right]^{N}},
$$

can be found similarly to the case of the original IBVP (7.1), (7.2) by exploiting the Fourier expansion of the solution of (7.13), (7.14) with respect to the system of eigenfunctions of the corresponding Galerkin eigenvalue problem (6.9), (6.10). The uniqueness can be proved once more by the energy method.

Translating these conservation laws with the help of identities (3.21) (with $\rho$ replacing $\rho_{0}$ and $M$ replacing $G_{0}$ ) and (3.11), we get (7.17) and (7.18); translating the mentioned eigenfunctions into the eigenfunctions of problem (6.6), we obtain (7.19).

Specific choices of $y^{(N), 0}$ are as follows.

1. The $\rho_{0}$-weighted $L^{2}(\Omega)$-projection of $\psi^{0}$ on $S_{N}$, i.e., $y^{(N), 0}=\mathcal{P}_{N} \psi^{0}$ satisfies the integral identity

$$
\int_{\Omega} y^{(N), 0} \varphi \rho_{0} d x=\int_{\Omega} \psi^{0} \varphi \rho_{0} d x \text { for any } \varphi \in S_{N} .
$$

In this case $\left\|\sqrt{\rho_{0}} y^{(N), 0}\right\|_{L^{2}(\Omega)} \leq\left\|\sqrt{\rho_{0}} \psi^{0}\right\|_{L^{2}(\Omega)}$ and

$$
G_{0} \mathbf{c}^{0}=\mathbf{g}_{0} \text { on } I_{1} \text {, with } g_{0 k}=\int_{I_{2}} \psi^{0} \chi_{k}^{*} \rho_{0} d x_{2} \text { for } 1 \leq k \leq N .
$$

2. The $\mathcal{E}(\Omega)$-projection of $\psi^{0}$ on $S_{N}$, i.e. $y^{(N), 0}=\mathcal{P}_{N}^{(1)} \psi^{0}$ satisfies the integral identity

$$
\mathcal{L}_{\Omega}\left(y^{(N), 0}, \varphi\right)=\mathcal{L}_{\Omega}\left(\psi^{0}, \varphi\right) \text { for any } \varphi \in S_{N} .
$$

In this case $\left\|y^{(N), 0}\right\|_{\mathcal{E}(\Omega)} \leq\left\|\psi^{0}\right\|_{\mathcal{E}(\Omega)}$ and

$$
\begin{aligned}
\mathcal{H}_{N} \mathbf{c}^{0} & =\mathbf{g}_{1} \quad \text { on } I_{1}, \\
\text { with } g_{1 k} & =\int_{I_{2}}\left(\sum_{i, j=1}^{2} \kappa_{i j} D_{j} \psi^{0} \cdot D_{i} \chi_{k}^{*}+V \psi^{0} \chi_{k}^{*}\right) d x_{2} \text { for } 1 \leq k \leq N, \\
\left.\mathbf{c}^{0}\right|_{x_{1}=0, X_{1}} & =0 .
\end{aligned}
$$

Here $\mathbf{g}_{m}=\left(g_{m 1}, \ldots, g_{m N}\right)^{T}$ for $m=0,1$.

We complete this last section by presenting error bounds in the case of the specific basis functions studied in section 4 . 
Proposition 7.4. Let conditions (4.20), (4.46) and (4.33) be valid. Also let

$$
\psi^{0} \in \mathbf{H}^{0, \theta}(\Omega), D_{1} \psi^{0} \in \mathbf{H}^{0, \theta-1}(\Omega), D_{t} \psi \in L^{1}\left(0, T ; \mathbf{H}^{0, \theta}(\Omega)\right),
$$

and

$$
D_{1} D_{t} \psi \in L^{1}\left(0, T ; \mathbf{H}^{0, \theta-1}(\Omega)\right),
$$

for some $\theta>1$ and, in the case where $\theta>3 / 2$, let condition (4.42) for $\beta=\theta-5 / 2$ be valid as well.

1. Let condition (2.8) be also valid. Then the following $C\left([0, T] ; L^{2}(\Omega)\right)$-error bound holds:

$$
\begin{aligned}
\sqrt{\underline{\rho}}\left\|\psi-y^{(N)}\right\|_{C\left([0, T] ; L^{2}(\Omega)\right) \leq} & \sqrt{\bar{\rho}}\left\|y^{(N), 0}-\mathcal{P}_{N}^{(1)} \psi^{0}\right\|_{L^{2}(\Omega)} \\
& +K(N+1)^{-\theta}(1+\log N)\left[\left\|\psi^{0}\right\|_{\mathbf{H}^{0, \theta}(\Omega)}+\left\|D_{1} \psi^{0}\right\|_{\mathbf{H}^{0, \theta-1}(\Omega)}\right. \\
& \left.+\left\|D_{t} \psi\right\|_{L^{1}\left(0, T ; \mathbf{H}^{0, \theta}(\Omega)\right)}+\left\|D_{1} D_{t} \psi\right\|_{L^{1}\left(0, T ; \mathbf{H}^{0, \theta-1}(\Omega)\right)}\right] .
\end{aligned}
$$

2. Let $\rho_{0}=\rho$. Then the following $C\left([0, T] ; H^{1}(\Omega)\right)$-error bound holds:

$$
\begin{aligned}
& \left\|\psi-y^{(N)}\right\|_{C([0, T] ; \mathcal{E}(\Omega))} \\
\leq & \left\|y^{(N), 0}-\mathcal{P}_{N} \psi^{0}\right\|_{\mathcal{E}(\Omega)} \\
& +K(N+1)^{-(\theta-1)}\left(1+\log ^{1 / 2} N\right)\left[\left\|\psi^{0}\right\|_{\mathbf{H}^{0, \theta}(\Omega)}+\left\|D_{1} \psi^{0}\right\|_{\mathbf{H}^{0, \theta-1}(\Omega)}\right. \\
& \left.+\left\|D_{t} \psi\right\|_{L^{1}\left(0, T ; \mathbf{H}^{0, \theta}(\Omega)\right)}+\left\|D_{1} D_{t} \psi\right\|_{L^{1}\left(0, T ; \mathbf{H}^{0, \theta-1}(\Omega)\right)}\right]
\end{aligned}
$$

Here $K$ is independent of $T$. For both specific choices (7.20) and (7.21) of $y^{(N), 0}$, the first summands can be omitted from the right hand sides of (7.22) and (7.23).

Proof. 1. The argument is rather standard in semi-discrete Galerkin methods for IBVP. Namely, for any $y$ with the properties like (7.8) and (7.9) of $y^{(N)}$, we have the following chain of identities following from the Galerkin and original integral identities (7.10) and (7.3):

$$
\begin{aligned}
& \imath\left\langle D_{t}\left[\rho\left(y^{(N)}-y\right)\right], \varphi\right\rangle_{\Omega}-\mathcal{L}_{\Omega}\left(y^{(N)}-y, \varphi\right) \\
= & \imath\left\langle D_{t}\left(\rho y^{(N)}\right), \varphi\right\rangle_{\Omega}-\mathcal{L}_{\Omega}\left(y^{(N)}, \varphi\right)-\left[\imath\left\langle D_{t}(\rho y), \varphi\right\rangle_{\Omega}-\mathcal{L}_{\Omega}(y, \varphi)\right] \\
= & \imath\left\langle D_{t}(\rho \psi), \varphi\right\rangle_{\Omega}-\imath\left\langle D_{t}(\rho y), \varphi\right\rangle_{\Omega}-\mathcal{L}_{\Omega}(\psi-y, \varphi) \text { on }(0, T), \text { for any } \varphi \in S_{N} .(7.24)
\end{aligned}
$$

Here the term $\imath\left\langle D_{t}\left(\rho y^{(N)}\right), \varphi\right\rangle_{\Omega}$ is actually understood as the left-hand side of identity $(7.15)$, and the terms $\imath\left\langle D_{t}\left[\rho\left(y^{(N)}-y\right)\right], \varphi\right\rangle_{\Omega}$ and $\imath\left\langle D_{t}(\rho y), \varphi\right\rangle_{\Omega}$ are understood similarly.

For $D_{t} \psi \in L^{1}\left(0, T ; H_{0}^{1}(\Omega)\right)$, setting $y:=\mathcal{P}_{N}^{(1)} \psi$ and $r^{(N)}:=y^{(N)}-\mathcal{P}_{N}^{(1)} \psi$ and using the Definition $(7.21)$ of $\mathcal{P}_{N}^{(1)}$, we have

$\imath\left\langle D_{t}\left(\rho r^{(N)}\right), \varphi\right\rangle_{\Omega}-\mathcal{L}_{\Omega}\left(r^{(N)}, \varphi\right)=\imath\left\langle D_{t}\left[\rho\left(\psi-\mathcal{P}_{N}^{(1)} \psi\right)\right], \varphi\right\rangle_{\Omega}$ on $(0, T)$, for any $\varphi \in S_{N}$.

Choosing $\varphi=r^{(N)}$ and separating the imaginary part of the result, we obtain

$$
\begin{aligned}
\frac{1}{2} \frac{d}{d t}\left\|\sqrt{\rho} r^{(N)}\right\|_{\Omega}^{2} & =\operatorname{Im}\left(\imath \int_{\Omega}\left(D_{t} \psi-\mathcal{P}_{N}^{(1)} D_{t} \psi\right)\left(r^{(N)}\right)^{*} \rho d x\right) \\
& \leq\left\|\sqrt{\rho}\left(D_{t} \psi-\mathcal{P}_{N}^{(1)} D_{t} \psi\right)\right\|_{\Omega}\left\|\sqrt{\rho} r^{(N)}\right\|_{\Omega} \text { on }(0, T)
\end{aligned}
$$


Consequently the following $C\left([0, T] ; L^{2}(\Omega)\right)$-bound for $r^{(N)}$ holds

$$
\left\|\sqrt{\rho} r^{(N)}\right\|_{C\left([0, T] ; L^{2}(\Omega)\right)} \leq\left\|\left.\sqrt{\rho} r^{(N)}\right|_{t=0}\right\|_{\Omega}+2\left\|\sqrt{\rho}\left(D_{t} \psi-\mathcal{P}_{N}^{(1)} D_{t} \psi\right)\right\|_{L^{1}\left(0, T ; L^{2}(\Omega)\right)} .
$$

This directly implies the corresponding error bound

$$
\begin{aligned}
\left\|\sqrt{\rho}\left(\psi-y^{(N)}\right)\right\|_{C\left([0, T] ; L^{2}(\Omega)\right)} \leq & \left\|\sqrt{\rho} r^{(N)}\right\|_{C\left([0, T] ; L^{2}(\Omega)\right)}+\left\|\sqrt{\rho}\left(\psi-\mathcal{P}_{N}^{(1)} \psi\right)\right\|_{C\left([0, T] ; L^{2}(\Omega)\right)} \\
\leq & \left\|\sqrt{\rho}\left(y^{(N), 0}-\mathcal{P}_{N}^{(1)} \psi^{0}\right)\right\|_{\Omega}+\left\|\sqrt{\rho}\left(\psi^{0}-\mathcal{P}_{N}^{(1)} \psi^{0}\right)\right\|_{\Omega} \\
& +3\left\|\sqrt{\rho}\left(D_{t} \psi-\mathcal{P}_{N}^{(1)} D_{t} \psi\right)\right\|_{L^{1}\left(0, T ; L^{2}(\Omega)\right)}
\end{aligned}
$$

Exploiting assumptions on $\psi^{0}$ and $D_{t} \psi$ as well as the $L^{2}(\Omega)$-error bound (4.47) for the BVP, we derive (7.22).

2. For $D_{t} \psi \in L^{1}\left(0, T ; H_{0}^{1}(\Omega)\right)$, now we set $y:=\mathcal{P}_{N} \psi$ and $q^{(N)}:=y^{(N)}-\mathcal{P}_{N} \psi$. In the case where $\rho_{0}=\rho$, using Definition (7.20) of $\mathcal{P}_{N}$, from (7.24) we obtain

$$
\imath\left\langle D_{t}\left(\rho q^{(N)}\right), \varphi\right\rangle_{\Omega}-\mathcal{L}_{\Omega}\left(q^{(N)}, \varphi\right)=-\mathcal{L}_{\Omega}\left(\psi-\mathcal{P}_{N} \psi, \varphi\right) \text { on }(0, T) \text {, for any } \varphi \in S_{N} .
$$

Supposing that the property $D_{t} y^{(N)} \in L^{1}\left(0, T ; H_{0}^{1}(\Omega)\right)$ is valid, then choosing $\varphi=$ $D_{t} q^{(N)}$ and separating the real part of the result, we obtain

$$
\frac{1}{2} \frac{d}{d t}\left(\left\|q^{(N)}\right\|_{\mathcal{E}(\Omega)}^{2}\right)=\operatorname{Re} \mathcal{L}_{\Omega}\left(\psi-\mathcal{P}_{N} \psi, D_{t} q^{(N)}\right) \text { on }(0, T) .
$$

Integrating this equality and then integrating by parts, we have

$$
\begin{aligned}
&\left\|q^{(N)}(\cdot, t)\right\|_{\mathcal{E}(\Omega)}^{2}=\left\|q^{(N), 0}\right\|_{\mathcal{E}(\Omega)}^{2}+2 \operatorname{Re}\left[\mathcal{L}_{\Omega}\left(\left(\psi-\mathcal{P}_{N} \psi\right)(\cdot, t), q^{(N)}(\cdot, t)\right)\right. \\
&\left.-\mathcal{L}_{\Omega}\left(\psi^{0}-\mathcal{P}_{N} \psi^{0}, q^{(N), 0}\right)-\int_{0}^{t} \mathcal{L}_{\Omega}\left(D_{t}\left(\psi-\mathcal{P}_{N} \psi\right), q^{(N)}\right) d \tau\right] \\
& \leq\left\|q^{(N), 0}\right\|_{\mathcal{E}(\Omega)}^{2}+2\left\|\psi^{0}-\mathcal{P}_{N} \psi^{0}\right\|_{\mathcal{E}(\Omega)}\left\|q^{(N), 0}\right\|_{\mathcal{E}(\Omega)} \\
&+2\left(\left\|\left(\psi-\mathcal{P}_{N} \psi\right)(\cdot, t)\right\|_{\mathcal{E}(\Omega)}+\left\|D_{t}\left(\psi-\mathcal{P}_{N} \psi\right)\right\|_{L^{1}(0, t ; \mathcal{E}(\Omega))}\right) \\
&\left\|q^{(N)}\right\|_{C([0, t] ; \mathcal{E}(\Omega))} \text { on }(0, T),
\end{aligned}
$$

where $q^{(N), 0}:=\left.q^{(N)}\right|_{t=0}=y^{(N), 0}-\mathcal{P}_{N} \psi^{0}$. Consequently

$$
\left\|q^{(N)}\right\|_{C([0, T] ; \mathcal{E}(\Omega))} \leq\left\|q^{(N), 0}\right\|_{\mathcal{E}(\Omega)}+3\left\|\psi^{0}-\mathcal{P}_{N} \psi^{0}\right\|_{\mathcal{E}(\Omega)}+4\left\|D_{t}\left(\psi-\mathcal{P}_{N} \psi\right)\right\|_{L^{1}(0, T ; \mathcal{E}(\Omega))} .
$$

To remove the temporary assumption $D_{t} y^{(N)} \in L^{1}\left(0, T ; H_{0}^{1}(\Omega)\right)$, we can once more apply the Fourier method based on expansions with respect to the system of eigenfunctions of the eigenvalue problem (6.9), (6.10). We rewrite identity (7.26) as an inhomogeneous equation like (7.13) for $q^{(N)}$, derive a bound like (7.27) for partial sums of the expansion for $q^{(N)}$, and then pass to the limit in the sums (see similar arguments, for example, in [13] and [26]).

Bound (7.27) implies the $C([0, T] ; \mathcal{E}(\Omega)$ )-error bound (compare with $(7.25)$ )

$$
\begin{aligned}
\left\|\psi-y^{(N)}\right\|_{C([0, T] ; \mathcal{E}(\Omega)) \leq} & \left\|y^{(N), 0}-\mathcal{P}_{N} \psi^{0}\right\|_{\mathcal{E}(\Omega)}+4\left\|\psi^{0}-\mathcal{P}_{N} \psi^{0}\right\|_{\mathcal{E}(\Omega)} \\
& +5\left\|D_{t} \psi-\mathcal{P}_{N} D_{t} \psi\right\|_{L^{1}(0, T ; \mathcal{E}(\Omega))}
\end{aligned}
$$


Exploiting assumptions on $\psi^{0}$ and $D_{t} \psi$ as well as the $H^{1}(\Omega)$-approximation bound (4.44), we obtain (7.23).

Since $\mathcal{P}_{N} \psi^{0}-\mathcal{P}_{N}^{(1)} \psi^{0}=\mathcal{P}_{N}\left(\psi^{0}-\mathcal{P}_{N}^{(1)} \psi^{0}\right)=-\mathcal{P}_{N}^{(1)}\left(\psi^{0}-\mathcal{P}_{N} \psi^{0}\right)$, for $y^{(N), 0}=\mathcal{P}_{N} \psi^{0}$ or $\mathcal{P}_{N}^{(1)} \psi^{0}$, the first terms on the right hand sides in (7.25) and (7.28) either can be bounded by the second ones or are simply zero. This completes the proof.

Finally we notice that our method can be also applied to second order parabolic or hyperbolic initial-boundary value problems although this seems beyond the scope of problems presently considered in nuclear physics.

Acknowledgments. The paper was mainly prepared during the visits of A. Zlotnik in autumns 2005 and 2007 to the Département de Physique Théorique et Appliquée of CEA/DAM/DIF which he thanks for hospitality. His research is also partially supported by the Russian Foundation for Basic Research, project nos. 07-0100416 and 08-01-90009-Bel and the Ministry of Education and Science of the Russian Federation, project no. 2.1.1/3276.

\section{REFERENCES}

[1] J.P. Aubin, Approximation of Elliptic Boundary Value Problems, Wiley-Interscience, New York, 1972.

[2] J.F. Berger, M. Girod and D. Gogny, Microscopic analysis of collective dynamics in low energy fission, Nuclear Physics, A 428, 23c-36c, 1984.

[3] J. Bergh and J. Löfstrom, Interpolation Spaces: An Introduction, Springer-Verlag, Berlin, 1976.

[4] C. Bernardi and M.-C. Pelissier, Spectral approximation of a Schrödinger type equation, Math. Models Meth. Appl. Sci., 4(1), 49-88, 1994.

[5] C. Bernardi, M. Dauge and Y. Maday, Spectral Methods for Axisymmetric Domains, Series in Applied Mathematics, Gauthier-Villars, Paris, 3, 1999.

[6] O.E. Besov, V.P. Il'in and S.M. Nikolskii, Integral Representations of Functions and Embedding Theorems, John Wiley \& Sons, New York etc., Vol. I, 1978.

[7] L.C. Evans, Partial Differential Equations, Amer. Math. Soc., Providence, R.I., 3rd ed., 2002.

[8] H. Goutte, J.F. Berger, P. Casoli and D. Gogny, Microscopic approach of fission dynamics applied to fragment kinetic energy and mass distributions in ${ }^{238} U$, Phys. Rev., C 71 , 024316-1-024316-13, 2005.

[9] J.J. Griffin and J.A. Wheeler, Collective motions in nuclei by the method of generator coordinates, Phys. Rev., 108, 311-327, 1957.

[10] B.Y. Guo, Spectral Methods and Their Applications, World Scientific, Singapore, 1998.

[11] D.L. Hill and J.A. Wheeler, Nuclear constitution and the interpretation of fission phenomena, Phys. Rev., 89, 1102-1145, 1953.

[12] H. Hofmann, Quantummechanical treatment of the penetration through a two-dimensional fission barrier, Nuclear Physics, A 224, 116-139, 1974.

[13] O.A. Ladyzhenskaya, The Boundary Value Problems of Mathematical Physics, Springer Verlag, New York etc., 1985.

[14] O.A. Ladyzhenskaya and N.N. Ural'tseva, Linear and Quasilinear Elliptic Equations, Academic Press, New York-London, 1968.

[15] O.A. Ladyzhenskaya, V.A. Solonnikov and N.N. Ural'tseva, Linear and Quasilinear Equations of Parabolic Type, Amer. Math. Soc., Providence, R.I., 1968.

[16] J. Libert, M. Girod and J.P. Delaroche, Microscopic description of superdeformed bands with Gogny force, Phys. Rev., C 60, 054301-1-054301-26, 1999.

[17] G.I. Marchuk and V.I. Agoshkov, An Introduction to Projective-Grid Methods, Nauka, Moscow, Russian, 1981.

[18] S.G. Mikhlin, The Numerical Performance of Variational Methods, Walters-Noordhof, Groningen, 1971.

[19] L. Pròchniak, P. Quentin, D. Samsoen and J. Libert, A self-consistent approach to the quadrupole dynamics of medium heavy nuclei, Nuclear Physics, A 730, 59-79, 2004.

[20] S.G. Rohozinski, J. Dobaczewski, B. Nerlo-Pomorska, K. Pomorski and J. Srebny, Microscopic dynamic calculations of collective states in Xenon and Barium isotopes, Nuclear Physics, A $292,66-87,1978$. 
[21] P. Ring, H. Hassman and J.O. Rasmussen, On the treatment of a two-dimensional fission model with complex trajectories, Nuclear Physics, A 296, 50-76, 1978.

[22] P. Ring and P. Schuck, The Nuclear Many-Body Problem, Springer-Verlag, New York, Heidelberg, Berlin, 1980.

[23] G. Strang and G. Fix, An Analysis of the Finite Element Method, Prentice-Hall Inc., Englewood Cliffs, N.J., 1973.

[24] A.A. Zlotnik, Coefficient stability of systems of differential equations, Diff. Equations, 20(2), 169-177, 1984.

[25] A.A. Zlotnik, Some finite-element and finite-difference methods for solving mathematical physics problems with non-smooth data in n-dimensional cube, Sov. J. Numer. Anal. Math. Modelling, 6(5), 421-451, 1991.

[26] A.A. Zlotnik, Convergence rate estimates of finite-element methods for second-order hyperbolic equations, Numerical Methods and Applications, G.I. Marchuk (ed.), CRC Press, 155-220, 1994. 